\title{
A Dielectrophilic Allenic Ketone-Enabled [4 + 2] Annulation with 3,3'-Bisoxindoles: Enantioselective Creation of Two Contiguous Quaternary Stereogenic Centers
}

Xiaodong Tang,,$^{, \dagger}$ Chuan Xiang Alvin Tan, ${ }^{\dagger} \neq$ Wai-Lun Chan, ${ }^{\dagger}$ Fuhao Zhang, ${ }^{\dagger,}$, Wenrui Zheng, ${ }^{\dagger,}$ Yixin $\mathrm{Lu}^{* \$, \dagger}, \pm, \circ$

$\S$ National University of Singapore (Suzhou) Research Institute, 377 Lin Quan Street, Suzhou Industrial Park, Suzhou, Jiangsu, 215123, China.

$\dagger$ Department of Chemistry, National University of Singapore, 3 Science Drive 3, Singapore, 117543 , Singapore.

† Graduate School for Integrative Sciences \& Engineering (NGS), National University of Singapore, 28 Medical Drive, Sin-gapore, 117456, Singapore.

\& Department of Chemistry, Southern University of Science and Technology, Shenzhen, 518000, China.

○ Joint School of National University of Singapore and Tianjin University, International Campus of Tianjin University, Binhai New City, Fuzhou 350207, China

*Email: chmlyx@,nus.edu.sg

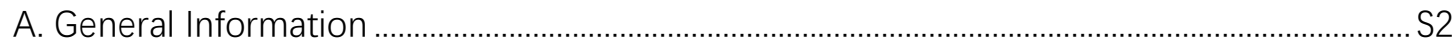

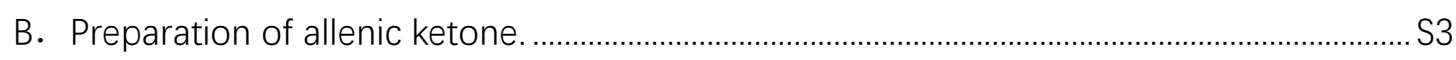

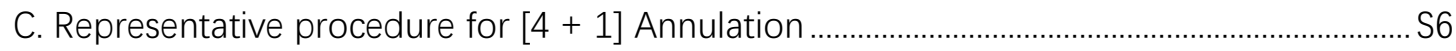

D. General procedure for $[4+2]$ annulation with bisoxindoles ................................................ S6

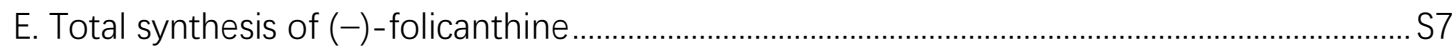

F. Mechanistic evidence: resting state of phosphine ............................................................... 10

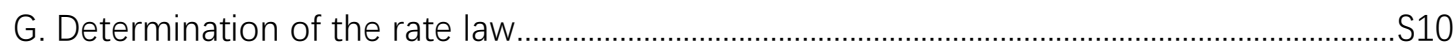

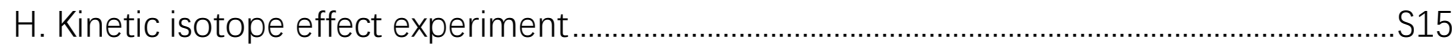

I. Analytical Data and HPLC Chromatograms of the Products..................................................S18

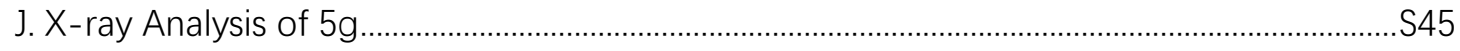

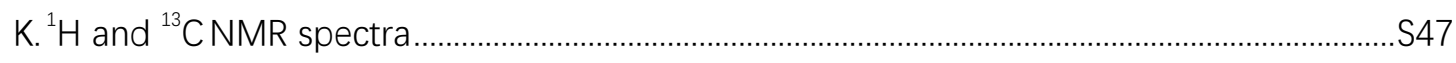

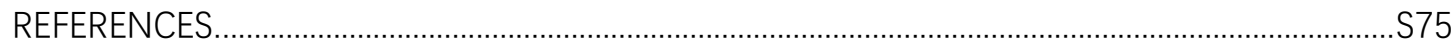




\section{A. General Information}

Unless otherwise specified, all reactions were conducted under an inert atmosphere and anhydrous conditions. All the solvents were purified according to the standard procedures. All chemicals which are commercially available were employed without further purification. Thin-layer chromatography (TLC) was performed on silica gel plates (60F-254) using UV-light (254 and $365 \mathrm{~nm})$. Flash chromatography was conducted on silica gel (200-300 mesh). ${ }^{1} \mathrm{H}$ and ${ }^{13} \mathrm{C}$ NMR spectra were recorded at ambient temperature in $\mathrm{CDCl}_{3}$ on a Bruker AMX500 (500 MHz) or AMX400 (400 $\mathrm{MHz}$ ) spectrometer. Chemical shifts were reported in parts per million ( $\mathrm{ppm}$ ). The data are reported as follows: for ${ }^{1} \mathrm{H} \mathrm{NMR}$, chemical shift in ppm from tetramethylsilane with the solvent as internal standard $\left(\mathrm{CDCl}_{3} \delta 7.26 \mathrm{ppm}\right)$, multiplicity $(\mathrm{s}=$ singlet, $\mathrm{d}=$ doublet, $\mathrm{t}=$ triplet, $\mathrm{q}=$ quartet, $\mathrm{m}=$ multiplet or overlap of non-equivalent resonances), integration; for ${ }^{13} \mathrm{C} \mathrm{NMR}$, chemical shift in ppm from tetramethylsilane with the solvent as internal indicator $\left(\mathrm{CDCl}_{3} \delta 77.1 \mathrm{ppm}\right)$, multiplicity with respect to protons . All highresolution mass spectra were performed by the MS service at the chemistry department, National University of Singapore, and were obtained on a Finnigan/MAT 95XL-T spectrometer to be given in m/z. Optical rotations were measured using an Anton Paar MCP-100 digital polarimeter using a $1 \mathrm{~cm}$ glass cell. Enantiomeric excesses were determined by HPLC analysis on a chiral stationary phase using CHIRALPAK ${ }^{\circledR}$ columns (IA and ID) eluting with hexane/isopropanol mixtures as indicated. Catalyst P5 was synthesized by following our previously reported procedures. ${ }^{1} \mathrm{~N}$-Protected Bisoxindoles 1 were synthesized according to the literature-reported procedures. ${ }^{2}$ 


\section{B. Preparation of allenic ketone}

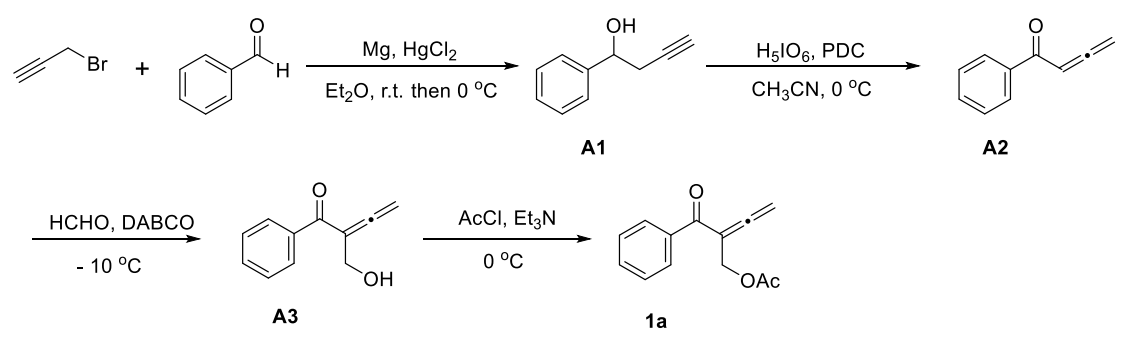

\section{$\underline{\text { 1-Phenylbut-3-yn-1-ol A1 }}$}

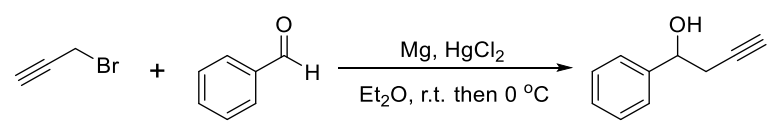

To a two-neck round bottom flask equipped with a condenser, $\mathrm{Et}_{2} \mathrm{O}(60 \mathrm{~mL})$, magnesium (642 mg, $26.4 \mathrm{mmol})$ and $\mathrm{HgCl}_{2}(54 \mathrm{mg}, 0.2 \mathrm{mmol})$ were mixed under $\mathrm{N}_{2}$. Then $80 \%$ propargyl bromide solution in toluene $(2.4 \mathrm{~mL}, 30 \mathrm{mmol})$ was added using a syringe through the rubber septum. The reaction mixture was stirred for 30 mins until complete disappearance of magnesium. Benzaldehyde (3.7 g, $20 \mathrm{mmol})$ was then added under $0{ }^{\circ} \mathrm{C}$, and the reaction was stirred 3 hours until the benzaldehyde was consumed completely. The reaction was then quenched by adding saturated ammonium chloride solution $(20 \mathrm{~mL})$ and extracted with $\mathrm{Et}_{2} \mathrm{O}(3 \times 30 \mathrm{~mL})$. The combined organic layers were washed with brine $(30 \mathrm{~mL})$ and dried over sodium sulfate. The organic layers were concentrated by rotary evaporation and purified using a flash column chromatography (hexane:ethyl acetate $=10: 1)$ to afford A1 as a yellowish oil (2.48 g, 85\% yield).

\section{1-Phenylbuta-2,3-dien-1-one A2}

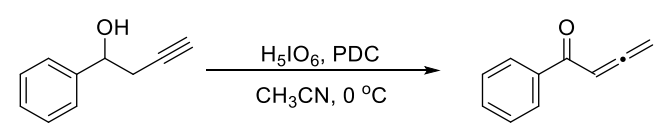

To a $250 \mathrm{~mL}$ round bottle flask charged with $\mathrm{H}_{5} \mathrm{IO}_{6}(5.2 \mathrm{~g}, 17.9 \mathrm{mmol})$ and $\mathrm{CH}_{3} \mathrm{CN}$ (120 mL), a solution of $\mathbf{A 1}(2.48 \mathrm{~g}, 17 \mathrm{mmol})$ in $\mathrm{CH}_{3} \mathrm{CN}(20 \mathrm{~mL})$ was added dropwisely under $0{ }^{\circ} \mathrm{C}$. Then, pyridinium chlorochromate $(128 \mathrm{mg}, 0.34 \mathrm{mmol})$ was added into the mixture in three portions during 5mins. After keep stirring for an hour, the reaction was monitored by TLC (Hexane:Ethyl acetate $=10: 1$ ) showed that all A1 had been consumed, then the ice bath was removed and the reaction was diluted with ethyl acetate 
$(50 \mathrm{~mL})$. The reaction mixture was washed with water $(100 \mathrm{~mL})$, separated organic phase was washed with saturated $\mathrm{Na}_{2} \mathrm{~S}_{2} \mathrm{O}_{3}(50 \mathrm{~mL})$, and brine $(50 \mathrm{~mL})$, dried over $\mathrm{Na}_{2} \mathrm{SO}_{4}$. After concentrating under reduced pressure, the crude product was purified by a column chromatography using hexane:ethyl acetate $=10: 1$ to afford $\mathbf{A 2}$ as an orange solid (1.49 g, 61\% yield).

For cyclohexane substrates 1q, Dess-Martin reagent was used as the only oxidant.

2-(Hydroxymethyl)-1-phenylbuta-2,3-dien-1-one A3

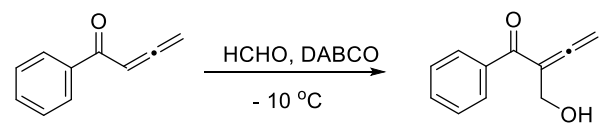

Formaldehyde solution was prepared using $33 \mathrm{~g}$ of powdered paraformaldehyde with $4 \mathrm{~mL} 1 \mathrm{~N}$ solution of $\mathrm{H}_{3} \mathrm{PO}_{4}$ in $100 \mathrm{~mL} \mathrm{H}_{2} \mathrm{O}$ and heated at $90{ }^{\circ} \mathrm{C}$ for 1 hour until all the paraformaldehyde has dissolved to give a clear solution.

To a round bottle flask $150 \mathrm{~mL}, \mathrm{DABCO}(235.5 \mathrm{mg}, 2.1 \mathrm{mmol})$ and $11 \mathrm{M}$ formaldehyde (4 mL, $52 \mathrm{mmol})$ dissolved in $80 \mathrm{~mL}$ THF at $-10{ }^{\circ} \mathrm{C}$. A2 (1.49 g, 10.4 $\mathrm{mmol}$ ) in $20 \mathrm{~mL}$ THF was added dropwise. The reaction was left to stir for $1 \mathrm{~h}$ under inert atmosphere and monitored by TLC (Hexane:Ethyl acetate $=2: 1$ ) until the $\mathbf{A 2}$ consumed completely. The reaction mixture was then quenched with saturated $\mathrm{NH}_{4} \mathrm{Cl}$ and the aqueous layer was extracted with ethyl acetate $(3 \times 40 \mathrm{~mL})$. The combined organic layers were washed with brine and dried over $\mathrm{Na}_{2} \mathrm{SO}_{4}$ before concentrated under reduced pressure. The crude product was then purified using a column chromatography (Hexane:Ethyl acetate $=2: 1)$ to yield $\mathbf{A 3}$ as an orange oil (1.27 g, 70\% yield).

\section{2-Benzoylbuta-2,3-dien-1-yl acetate 1a}

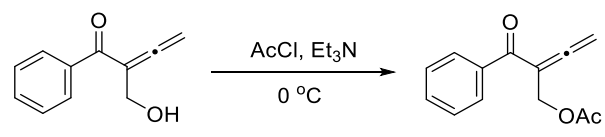

To a round bottle flask $100 \mathrm{~mL}, \mathbf{A 3}(1.27 \mathrm{~g}, 7.29 \mathrm{mmol})$ was dissolved in $20 \mathrm{~mL}$ $\mathrm{CH}_{2} \mathrm{Cl}_{2}$ at $0{ }^{\circ} \mathrm{C}$, and acetyl chloride $(680 \mathrm{mg}, 8.68 \mathrm{mmol})$ was added in one portion. Triethylamine $(876.6 \mathrm{mg}, 8.68 \mathrm{mmol})$ was then added dropwise. After $5 \mathrm{~min}$ with TLC confirmation that all the $\mathbf{A 3}$ has reacted, saturated $\mathrm{NH}_{4} \mathrm{Cl}$ was added to quench the 
reaction. The aqueous layer was then extracted using $\mathrm{CH}_{2} \mathrm{Cl}_{2}(3 \times 20 \mathrm{~mL})$. The combined organic layers were washed using brine and dried over $\mathrm{Na}_{2} \mathrm{SO}_{4}$ before concentrated under reduced pressure. The crude product was then purified using a column chromatography (Hexane:Ethyl acetate $=20: 1)$ to yield 1a as a yellow oil (629 mg, 40\% yield). 


\section{Representative procedure for $[4+1]$ Annulation}
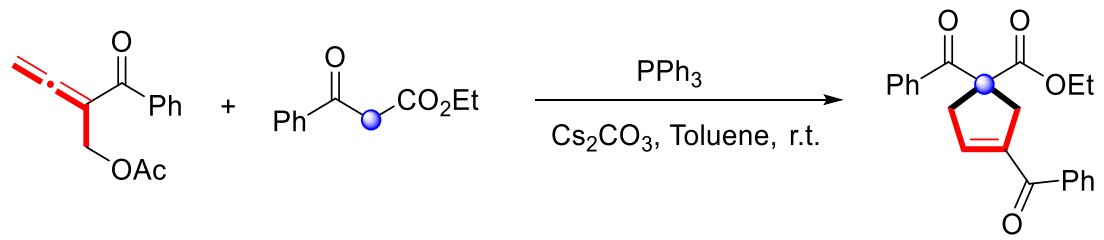

To a $4 \mathrm{~mL}$ sealed reaction vial, cesium carbonate $(32.5 \mathrm{mg}, 0.1 \mathrm{mmol})$ and $\mathrm{PPh}_{3}$ (5.2 $\mathrm{mg}, 0.02 \mathrm{mmol})$ and ethyl benzoylacetate $(19.2 \mathrm{mg}, 0.1 \mathrm{mmol})$ was added to 2.0 $\mathrm{mL}$ of toluene, then 2-benzoylbuta-2,3-dien-1-yl acetate (26 $\mathrm{mg}, 0.12 \mathrm{mmol}$ ) was injected slowly into the stirring solution using a micro-syringe. The reaction vial was left to stir at room temperature until the allene has been fully consumed. The solution was then purified using a column chromatography (Hexane:Ethyl acetate $=20: 1)$ to yield a yellow solid as the cyclized product (33.4 $\mathrm{mg}, 96 \%$ yield).

\section{General procedure for [4 + 2] annulation with bisoxindoles}

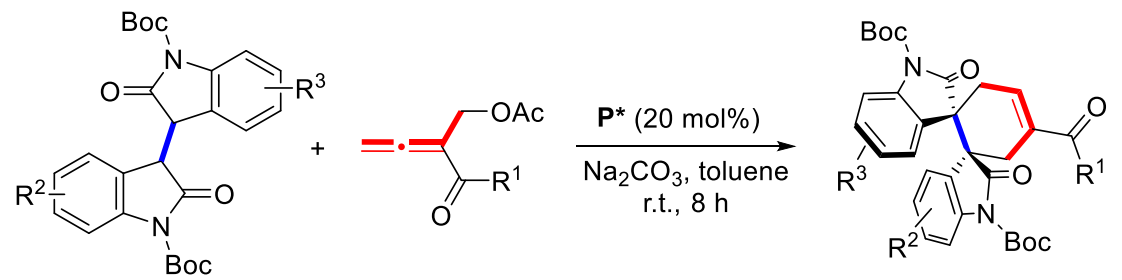

To a dry Schlenk tube with a magnetic bar, bisoxindoles $(0.12 \mathrm{mmol}, 1.2 \mathrm{eq}$.), sodium carbonate (0.12 mmol, 1.2 eq.) and chiral phosphine catalyst $\mathbf{P} *(0.02 \mathrm{mmol}$, 0.2 eq. $)$ was added into toluene $(2.0 \mathrm{~mL})$ at room temperature. Then allenic ketone $(0.1$ mmol, 1.0 eq.) was added slowly using a micro-syringe. The reaction vial was left to stir for 8 hours. The solution was then purified using a column chromatography (Hexane:Ethyl acetate $=20: 1)$ to yield the $[4+2]$ annulation product. 


\section{E. Total synthesis of (-)-folicanthine}

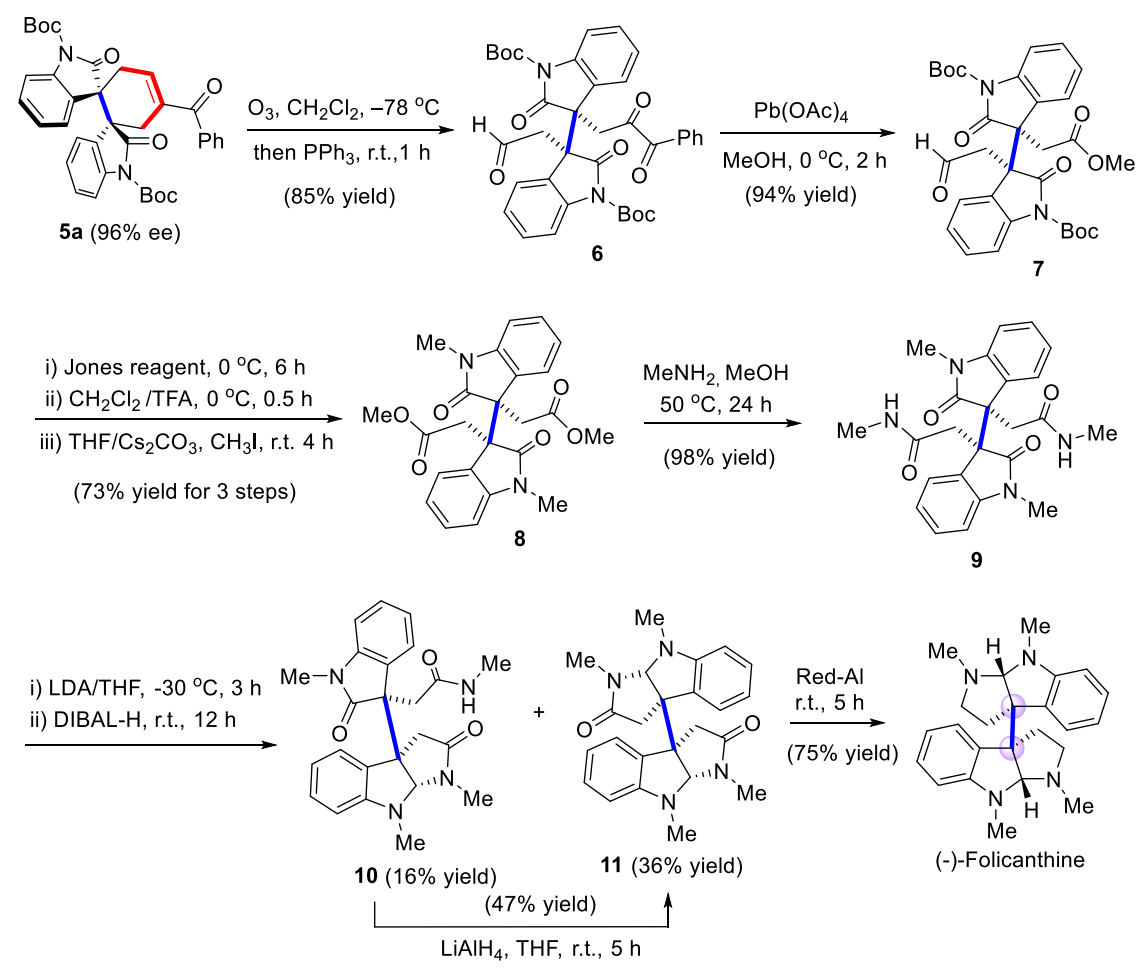

For compound 6: In a $250 \mathrm{~mL}$ round bottle flask, a solution of 5a $(310 \mathrm{mg}$; 0.5 $\mathrm{mmol})$ in $\mathrm{CH}_{2} \mathrm{Cl}_{2}(60 \mathrm{~mL})$ was cooled to $-78^{\circ} \mathrm{C}$. Ozone $\mathrm{O}_{3}$ was then bubbled through the solution for about 20 mins until the $\mathbf{5 a}$ was completely consumed. The flow of ozone was stopped, and $\mathrm{O}_{2}$ was bubbled through the solution until the blue color disappeared. After being stirred at this temperature for 30 mins, the reaction mixture was warmed to room temperature and $\mathrm{PPh}_{3}(196.5 \mathrm{mg}, 0.75 \mathrm{mmol})$ was added. After 1 hour, the solvent was removed under reduced pressure. The residue mixture was purified by a column chromatography (Hexane:Ethyl acetate $=4: 1)$ to yield a grey oil $(284 \mathrm{mg}, 87 \%$ yield $)$.

The product (287 mg, $0.43 \mathrm{mmol}$ ) obtained from ozonolysis was dissolved in a 50 $\mathrm{mL}$ round bottle flask with anhydrous methanol $(10.0 \mathrm{~mL})$, and lead tetraacetates $(288.6$ $\mathrm{mg}, 0.65 \mathrm{mmol}$ ) was added in one portion at $0{ }^{\circ} \mathrm{C}$. The reaction mixture was stirred under this temperature for 2 hours. The reaction mixture was filtered with celite and washed with ethyl acetate. Remove the organic solvent under vacuum, the residue was purified by a column chromatography (Hexane:Ethyl acetate $=4: 1)$ to yield a white solid $(233.6$ mg, 94\% yield). 
To a solution of 7 in acetone $(10 \mathrm{~mL})$ at $0{ }^{\circ} \mathrm{C}$, excess mount Jones reagent was added until the reaction remained orange color. Keep stirring for 2 hours, then the reaction was quenched by adding $i$-propanol until the color turned to green. Removing the solvent under vacuum, the residue was dissolved in water and was extracted with $\mathrm{CH}_{2} \mathrm{Cl}_{2}(20 \mathrm{~mL})$ three times, following wash with brine and dried over with $\mathrm{Na}_{2} \mathrm{SO}_{4}$. After removing the organic solvent under vacuum, the cured product was used without further purification. To a $\mathrm{CH}_{2} \mathrm{Cl}_{2}(4 \mathrm{~mL})$ solution of the crude product in round bottle flask under ice bath, trifluoroacetic acid $(0.4 \mathrm{~mL})$ was added. Keep stirring for half an hour, the reaction mixture was work up with rotavapor to dryness. Anhydrous THF (8 $\mathrm{mL}), \mathrm{Cs}_{2} \mathrm{CO}_{3}(130 \mathrm{mg}, 0.4 \mathrm{mmol})$ and $\mathrm{MeI}(568 \mathrm{mg}, 4 \mathrm{mmol})$ was added to the residue under nitrogen atmosphere. The reaction mixture was stirred for 4 hours under room temperature. Removing organic solvent, extracted with ethyl acetate three times. Purified by a column chromatography (Hexane:Ethyl acetate $=4: 1$ ) to yield a white solid (128.6 mg, 73\% yield for 3 steps).

A $15 \mathrm{~mL}$ solution of $40 \%$ methyl amine in methanol was added to a round bottle flash containing $8(128 \mathrm{mg}, 0.29 \mathrm{mmol})$ at room temperature. The solution was heated to $60{ }^{\circ} \mathrm{C}$ for 24 hours. The reaction mixture was dried under vacuum and was purified with column $\left(\mathrm{CH}_{2} \mathrm{Cl}_{2}\right.$ :Methanol $\left.=20: 1\right)$ to get the formal intermediate $9(125.5 \mathrm{mg}, 98 \%$ yield). ${ }^{3}$

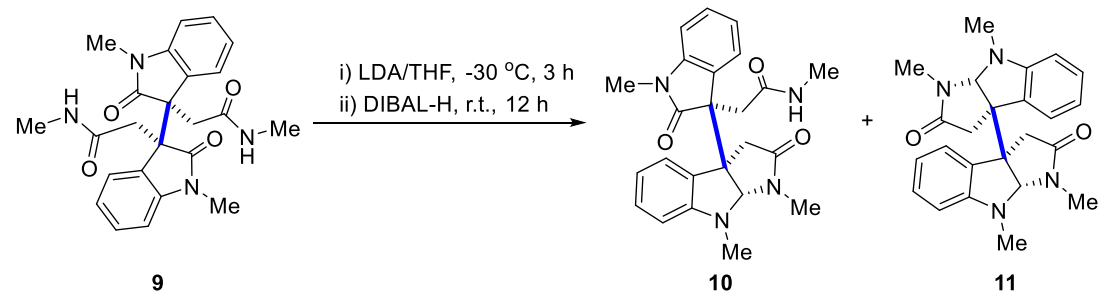

To a suspension of $9(130 \mathrm{mg}, 0.30 \mathrm{mmol})$ in fresh distilled THF $(80 \mathrm{~mL})$ at $-10{ }^{\circ} \mathrm{C}$ was added a $1 \mathrm{M}$ solution of LDA $(0.9 \mathrm{~mL}, 0.90 \mathrm{mmol})$. The mixture was stirred at -10 ${ }^{\circ} \mathrm{C}$ for 1 hour and then at room temperature for an additional 1 hour. The reaction mixture was cooled to $-30{ }^{\circ} \mathrm{C}$ and $2 \mathrm{M}$ diisobutylaluminum hydride $(1.5 \mathrm{~mL}, 3.0 \mathrm{mmol})$ was added with a syringe and then stirred for 24 hours at room temperature until the starting materials disappeared. Saturated sodium tartrate $(20 \mathrm{~mL})$ was added into the 
mixture and stirred vigorously until the organic phase becomes clear, separate the organic phase, and the aquas phase was extracted with ethyl acetate. The combined extracts were washed with brine, dried with $\mathrm{Na}_{2} \mathrm{SO}_{4}$ and concentrated under vacuum. The residue was dissolved in $\mathrm{CH}_{2} \mathrm{Cl}_{2}(10 \mathrm{~mL})$ treated with 10 -camphorsulfonic acid (20 $\mathrm{mg}, 0.08 \mathrm{mmol}$ ) and stirred overnight at room temperature. The solution was washed with $5 \%$ aqueous $\mathrm{NaHCO}_{3}$ and dried over $\mathrm{Na}_{2} \mathrm{SO}_{4}$. After concentration under reduced pressure. The residue obtained was purified by a column chromatography $\left(\mathrm{CH}_{2} \mathrm{Cl}_{2}\right.$ : Methanol $=30: 1)$ to afford the cyclization product 11 (43 $\mathrm{mg} \mathrm{36 \%} \mathrm{yield)} \mathrm{and} \mathrm{semi-}$ cyclization product $\mathbf{1 0}(20 \mathrm{mg}, 16 \%$ yield $)$.

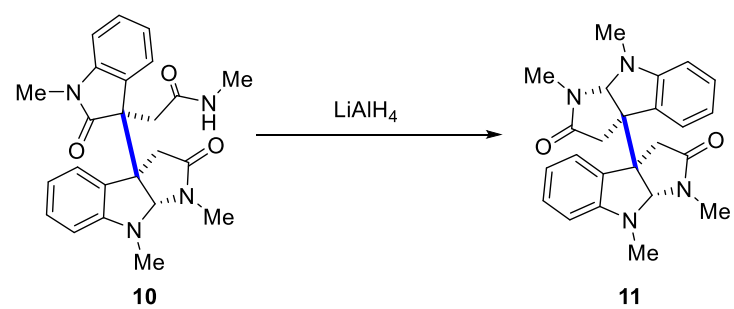

To a suspension of $\mathrm{LiAlH}_{4}(3 \mathrm{mg}, 0.07 \mathrm{mmol})$ in fresh distilled THF $(1.0 \mathrm{~mL})$ at 0 ${ }^{\circ} \mathrm{C}$ was added a solution of $\mathbf{1 0}(30 \mathrm{mg}, 0.024 \mathrm{mmol})$ in fresh distilled THF $(0.4 \mathrm{~mL})$ via syringe. The resulting mixture was stirred at room temperature for $5 \mathrm{~h}$ and quenched with $\mathrm{Na}_{2} \mathrm{SO}_{4} \cdot 10 \mathrm{H}_{2} \mathrm{O}$. After concentration under reduced pressure, the residue obtained was purified by a column chromatography (at $\mathrm{CH}_{2} \mathrm{Cl}_{2}$ :Methanol $=30: 1$ ) to afford compound 11 (15.0 mg, 47\%).

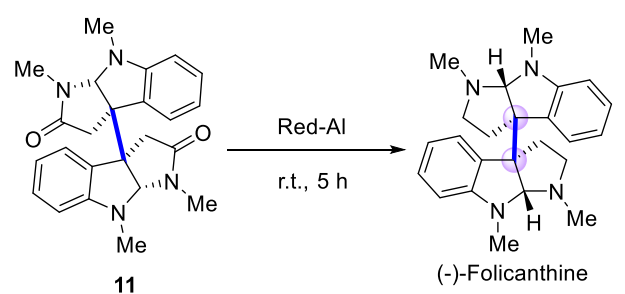

To a solution of $11(30 \mathrm{mg})$ in $2.0 \mathrm{~mL}$ toluene, $(308 \mathrm{mg}, 1.0 \mathrm{mmol})$ vitride (Red-Al $70 \%$ in toluene solution) was added at room temperature and stirred for 5 hours, and then quenched with $1.0 \mathrm{~mL}$ acetone. The mixture was extracted twice with ethyl acetate. The combined extracts were washed with brine and dried with $\mathrm{Na}_{2} \mathrm{SO}_{4}$. After concentration under reduced pressure, the residue obtained was purified by a column chromatography $\left(\mathrm{CH}_{2} \mathrm{Cl}_{2}\right.$ : methanol $=60: 1$, saturated with ammonia $)$ to afford the title 
compound (-)-folicanthine (20.1 mg, 75\%). Overall yield 18.7\%.

\section{F. Mechanistic evidence: resting state of phosphine}

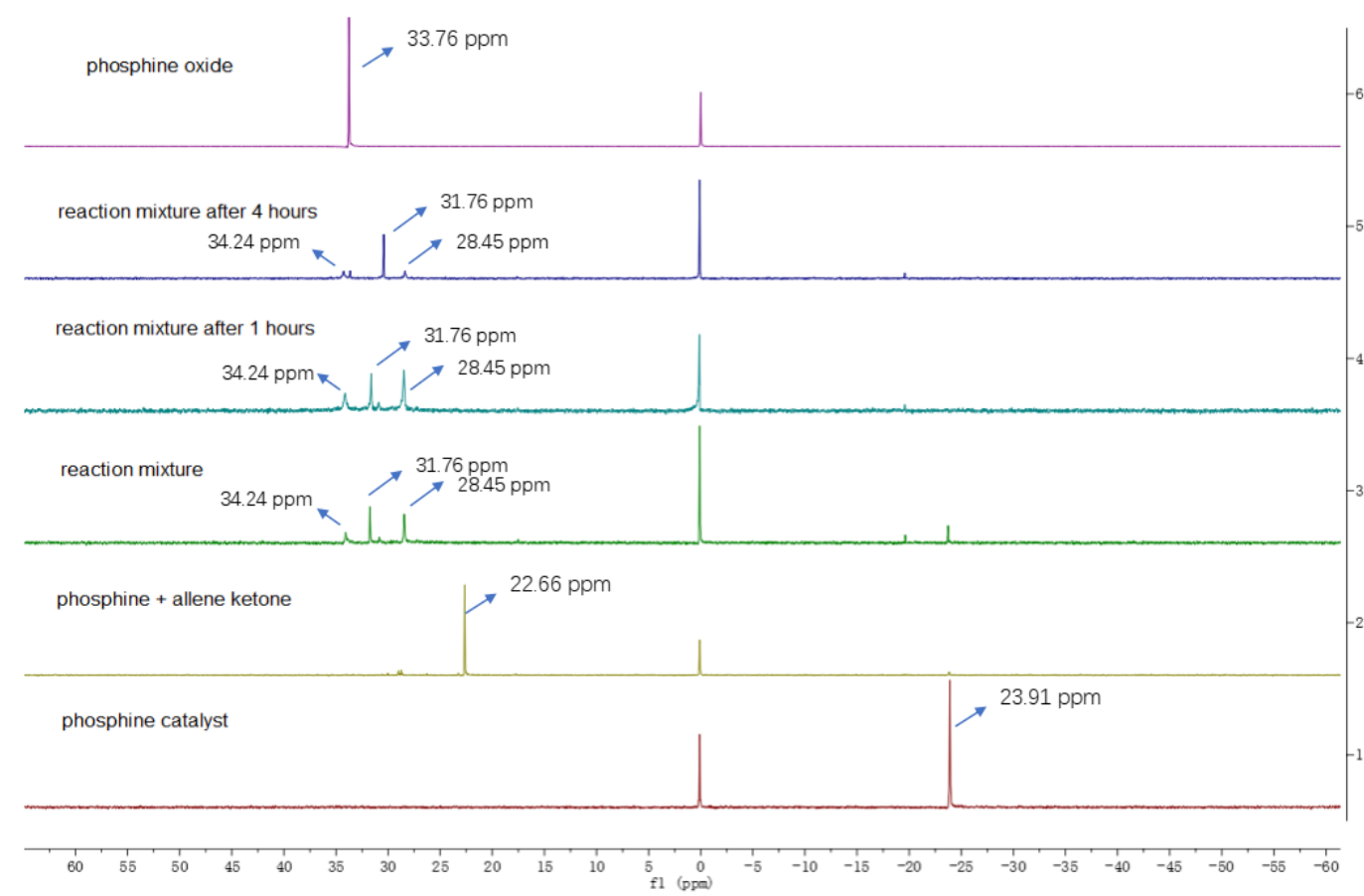

All the ${ }^{31}$ PNMR test were run in D8-toluene solution. A sealed capillary tube, containing a solution of $\mathrm{H}_{3} \mathrm{PO}_{4}\left({ }^{31} \mathrm{P}\right.$ NMR external standard; $2 \% \mathrm{w} / \mathrm{w}$ in $\left.\mathrm{D}_{2} \mathrm{O}\right)$, was inserted into the NMR tube.

In an $4 \mathrm{~mL}$ oven-dried vial, phosphine catalyst ( $0.01 \mathrm{mmol}, 0.2$ equiv. ), bisoxindole ( $0.6 \mathrm{mmol}, 1.2$ equiv.) and sodium carbonate( $0.6 \mathrm{mmol}, 1.2$ equiv.) were added to D8toluene solution $(0.5 \mathrm{~mL})$, then allenic ketone ( $0.5 \mathrm{mmol}, 1.0$ equiv.) was added in one portion under room temperature. The reaction mixture was taken from the vial for ${ }^{31}$ PNMR test every 0.5 hours for first 4 hours and every 1 hour for the next 3 hours.

Phosphine oxide was prepared by adding tert-butyl hydroperoxide (5 $\mathrm{M}$ in decane; $10 \mu \mathrm{L})$ to a solution of phosphine catalyst in D8-toluene.

\section{G. Determination of the rate law.}

The rate law was determined by the method of initial rates, following the formation of product up to $\sim 30 \%$ conversion of the limiting reagent/formation of the product. The reactions were carried out with a total volume of $1 \mathrm{~mL}$ (neglecting the volume of the 
internal standard). In an oven-dried vial, appropriate amounts of toluene (anhydrous) stock solutions of allenic ketone $(0.10 \mathrm{M})$, chiral phosphine P5 (0.03 M), bisoxindole $(0.50 \mathrm{M})$, and sodium carbonate (anhydrous) were mixed. Next, 1,3,5trimethoxybenzene (internal standard; $16.8 \mathrm{mg}, 0.1 \mathrm{mmol}$ ) was added, and the mixture was keep stirring at room temperature. Aliquots $(100 \mu \mathrm{L})$ were taken from the reaction mixture every 2 mins over a 10 mins period and were immediately quenched by addition of tert-butyl hydroperoxide ( $5 \mathrm{M}$ in decane; $10 \mu \mathrm{L}$ ). After the aliquots were concentrated under reduced pressure, the composition of each of the aliquots was determined by $1 \mathrm{H}$ NMR analysis.

Order in allenoate. Initial concentrations: $30.0 \mathrm{mM}( \pm 0.5 \mathrm{mM})$ in phosphine catatlyst; $100 \mathrm{mM}( \pm 1 \mathrm{mM})$ in sodium carbonate; $200 \mathrm{mM}( \pm 10 \mathrm{mM})$ in bisoxindole; $50-200 \mathrm{mM}$ in allenic ketone.

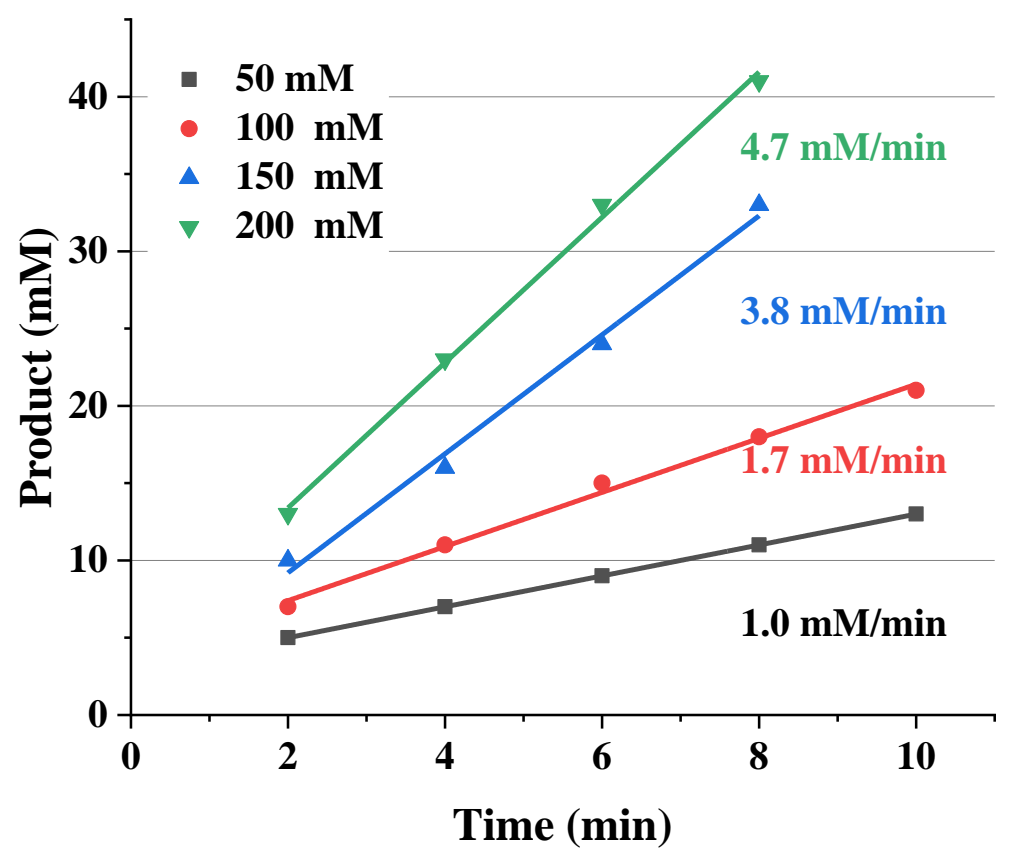




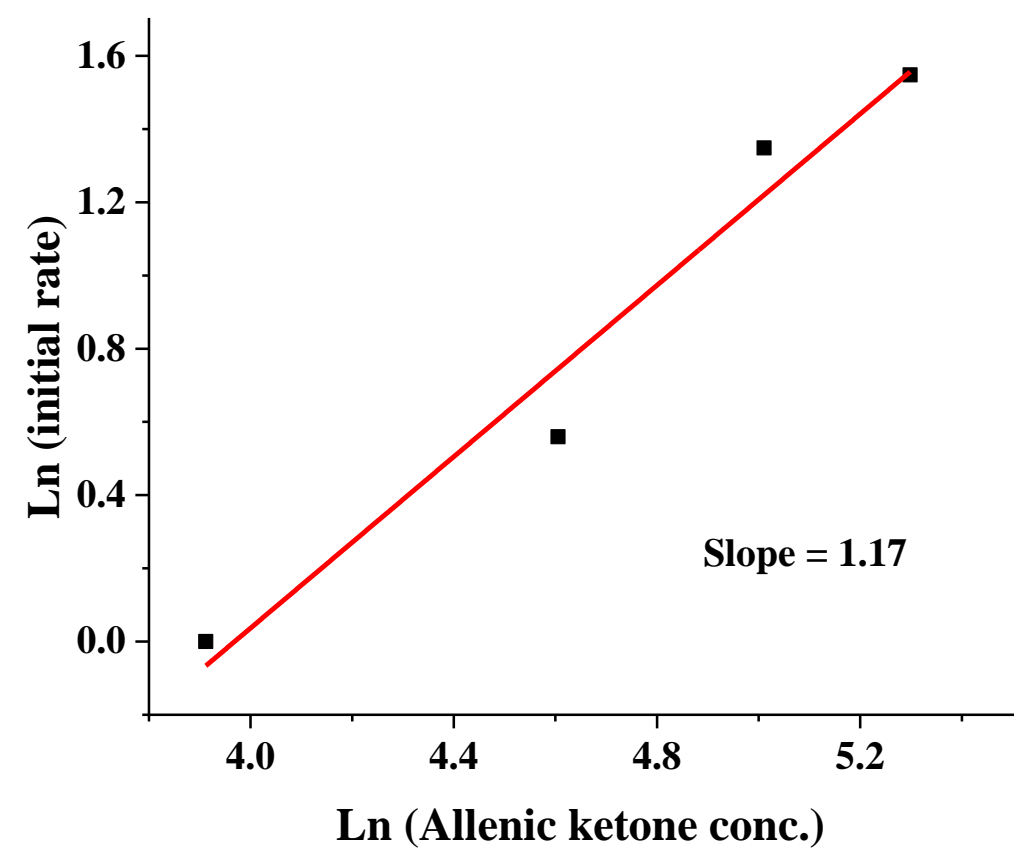

Order in bisoxindole. Initial concentrations: $30.0 \mathrm{mM}( \pm 0.5 \mathrm{mM})$ in phosphine catalyst; $100 \mathrm{mM}( \pm 1 \mathrm{mM})$ in sodium carbonate; $50-200 \mathrm{mM}$ in bisoxindole; $200 \mathrm{mM}( \pm 1 \mathrm{mM})$ in allenoate.

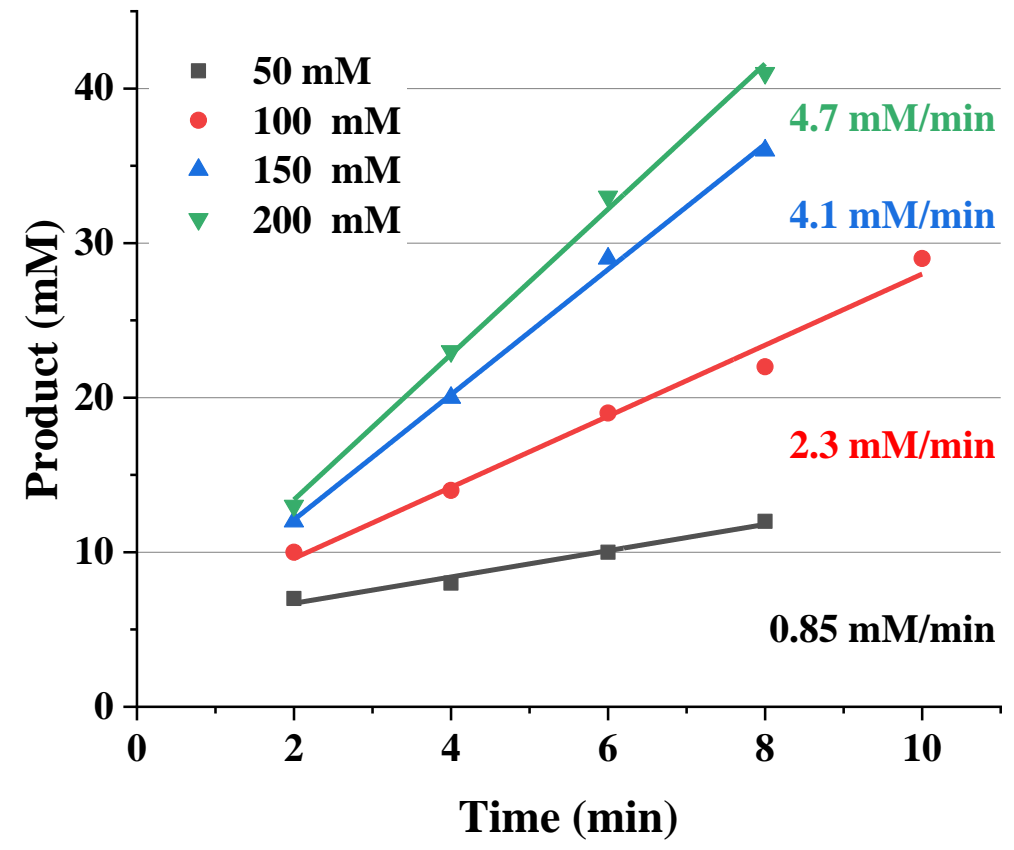




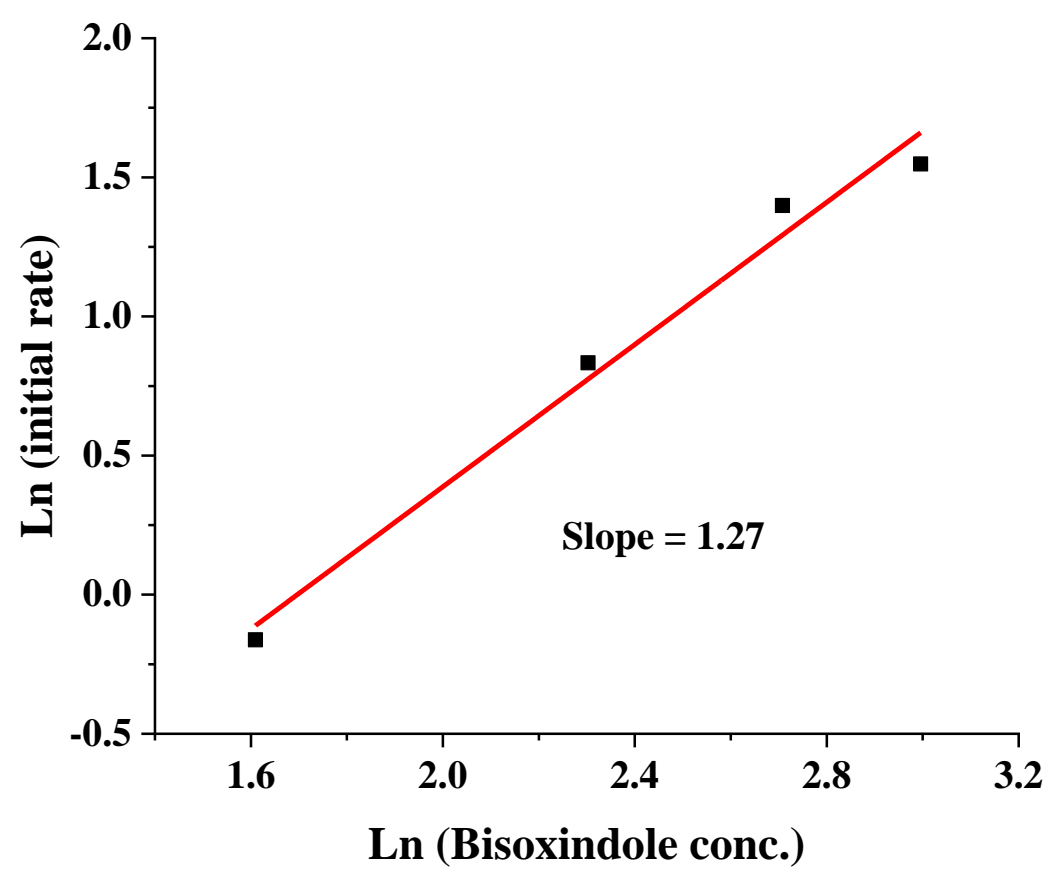

Order in catalyst. Initial concentrations: $10-40 \mathrm{mM}( \pm 0.1 \mathrm{mM})$ in phosphine catalyst; $200 \mathrm{mM}( \pm 1 \mathrm{mM})$ in sodium carbonate; $200 \mathrm{mM}( \pm 1 \mathrm{mM})$ in nucleophile; $100 \mathrm{mM}( \pm 1$ $\mathrm{mM})$ in allenoate. 

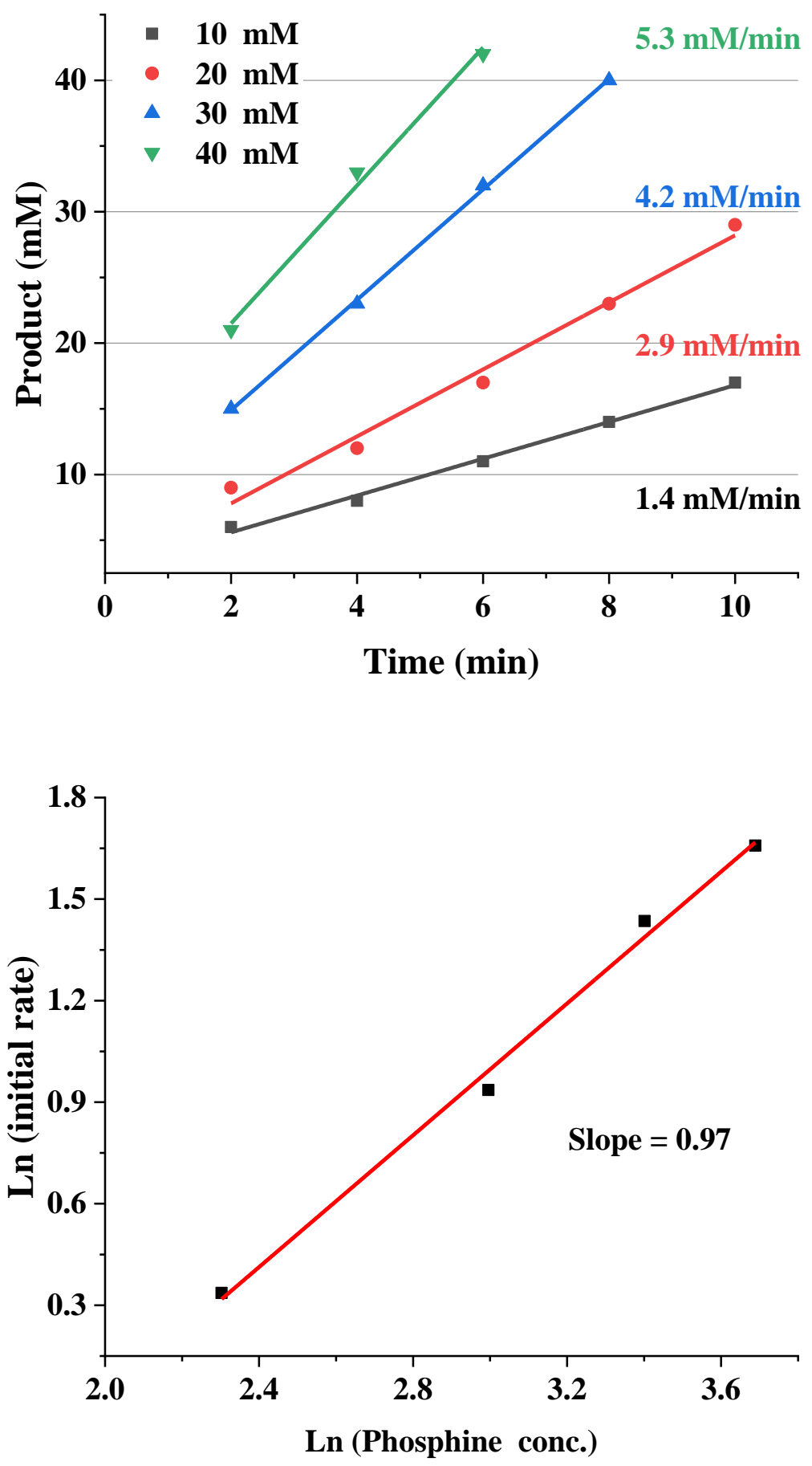


\section{H. Kinetic isotope effect experiment}

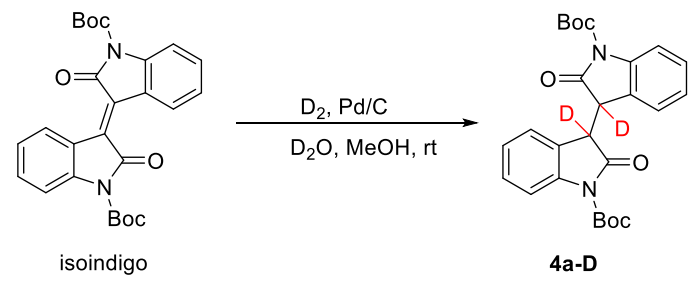

1. Synthesis of deuterated Bisoxindole:

$\mathrm{D}_{2}$ was generated according a modified process $^{[5]}: 20 \% \mathrm{Pd} / \mathrm{C}(10 \mathrm{wt} \%$ of the substrate, Sigma-Aldrich Co.) and $\mathrm{D}_{2} \mathrm{O}(5 \mathrm{~mL})$ was set in a $100 \mathrm{~mL}$ round-bottom flask (actual internal volume of the flask is $130 \mathrm{~mL}$ ). The system was sealed with a septum and filled with $\mathrm{H}_{2}$ by five vacuum/ $\mathrm{H}_{2}$ cycles. The mixture was stirred at room temperature for $24 \mathrm{~h}$. Then a solution of Isoindigo $(0.8 \mathrm{mmol})$ in $\mathrm{MeOH}(1 \mathrm{~mL})$ and THF ( $1 \mathrm{~mL}$ ) was added to the $\mathrm{D}_{2}$ filled flask. The mixture was stirred at room temperature for $6 \mathrm{~h}$, diluted with $\mathrm{Et}_{2} \mathrm{O}(10 \mathrm{~mL})$, and passed through a thin silica gel filter. The filtrate was separated into two layers. The aqueous layer was extracted with $\mathrm{Et}_{2} \mathrm{O}(2 \times 20 \mathrm{~mL})$ and the combined organic layers were washed with brine $(30 \mathrm{~mL})$, dried over $\mathrm{Na}_{2} \mathrm{SO}_{4}$, filtered, and concentrated under vacuum. The substrate then used without further purification. (silica gel column lead to deuterium lost) 


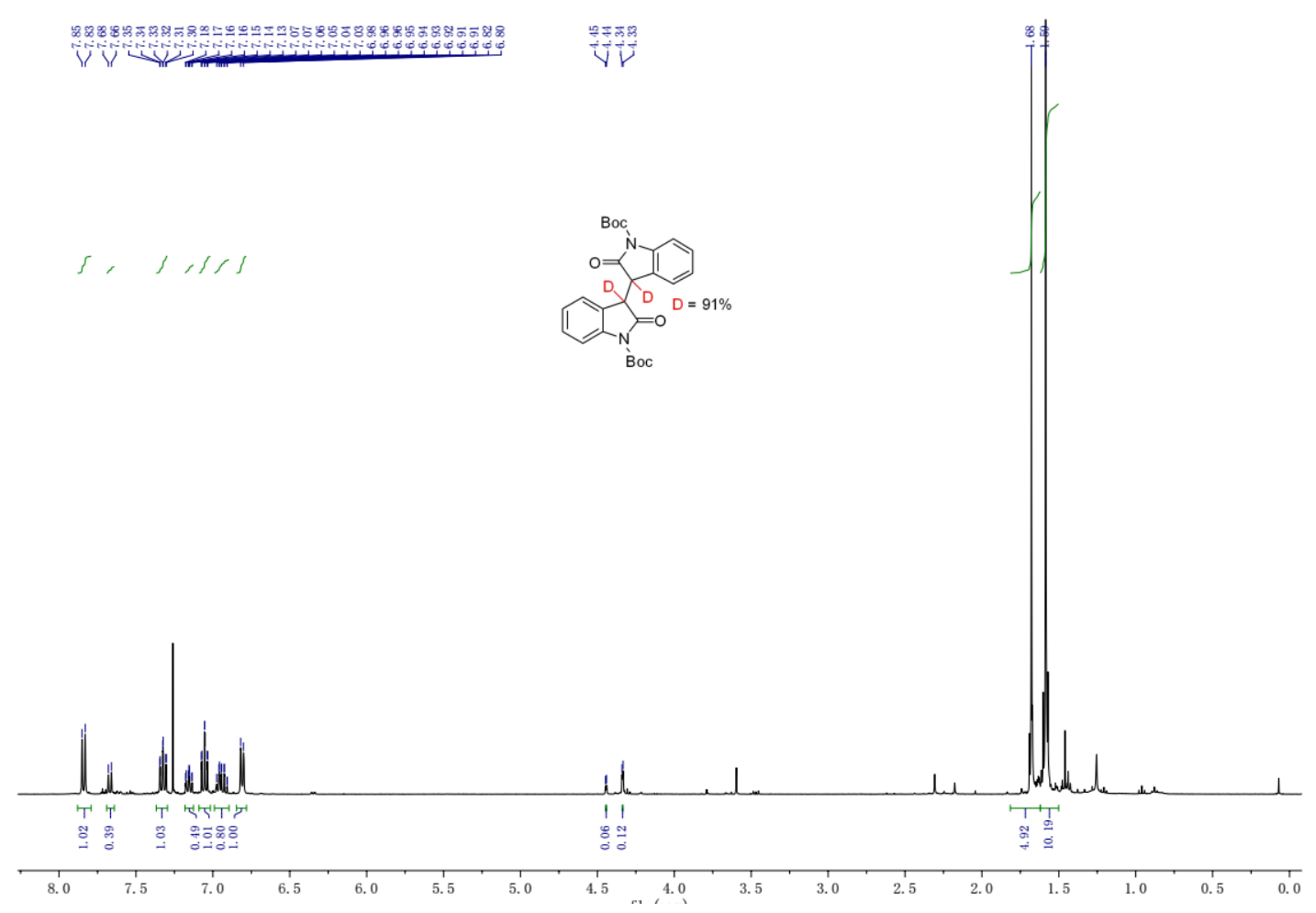

2. Monitoring rates of reactions using $\mathbf{4 a}$ and $\mathbf{4 a - D}$ as substrates

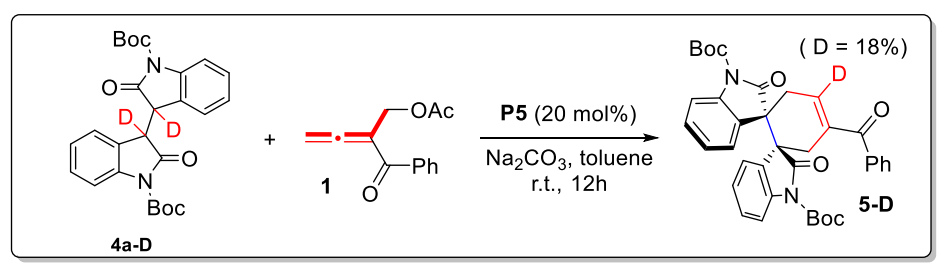

The rates of racemic Bisoxindole 4a versus racemic deuterated Bisoxindole 4a-D were determined as follows. To two vials ( $2 \mathrm{~mL}$, labelled " $\mathrm{H}$ " and " $\mathrm{D}$ ") were added 10 $\mathrm{mM}( \pm 0.1 \mathrm{mM})$ in phosphine catalyst P5; $200 \mathrm{mM}( \pm 1 \mathrm{mM})$ in sodium carbonate; 200 $\mathrm{mM}( \pm 1 \mathrm{mM})$ in Bisoxindole; $100 \mathrm{mM}( \pm 1 \mathrm{mM})$ in allenoate, 1,3,5-trimethoxybenzene (internal standard; $16.8 \mathrm{mg}, 0.1 \mathrm{mmol}$ ) was added, and the mixture was keep stirring at room temperature. Aliquots $(50 \mu \mathrm{L})$ were taken from the reaction mixture for every 2 min over a 10 min period and were immediately quenched by addition into other vials that contained a solution of tert-butyl hydroperoxide (5.0-6.0 M in decane, $5 \mu \mathrm{L})$. After the aliquots were concentrated under reduced pressure, the composition of each of the aliquots was determined by ${ }^{1} \mathrm{H}$ NMR analysis. Keep the reaction stirring until the starting materials disappeared, and purify the final product with column (Hexane : EA $=20: 1)$. 


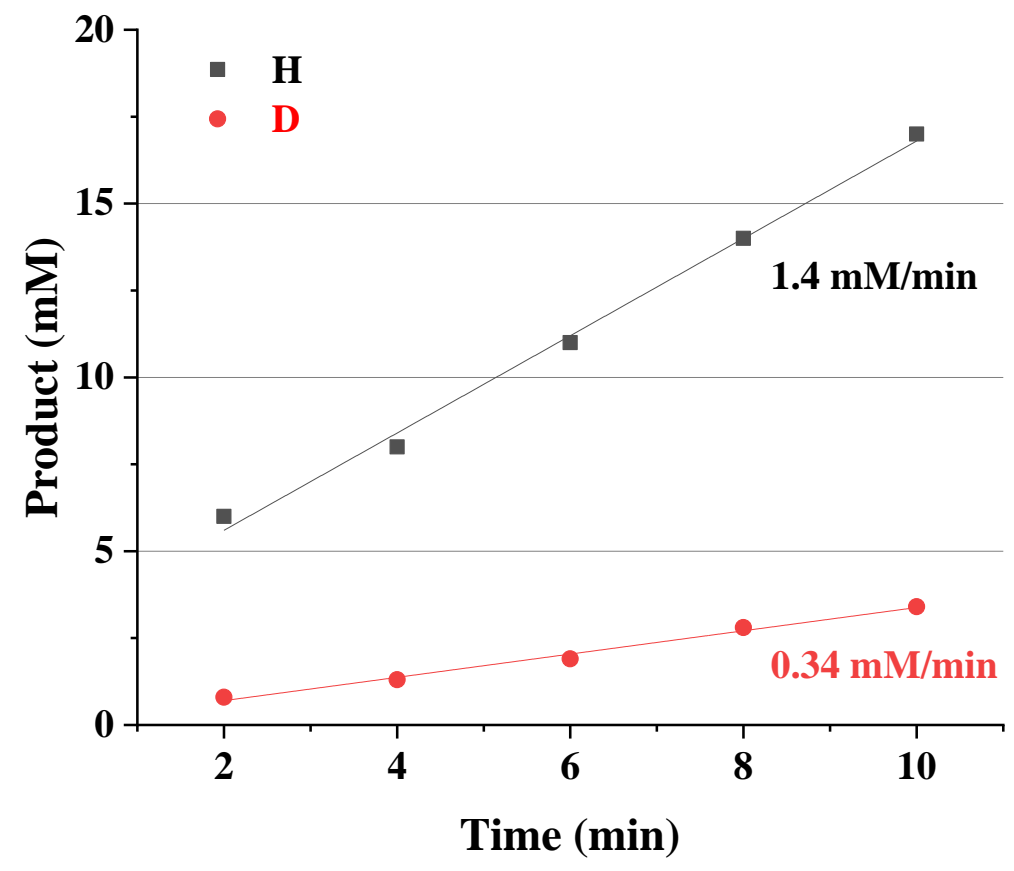

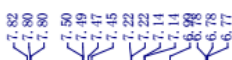

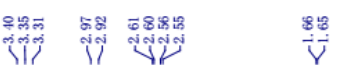

$1 / 111$
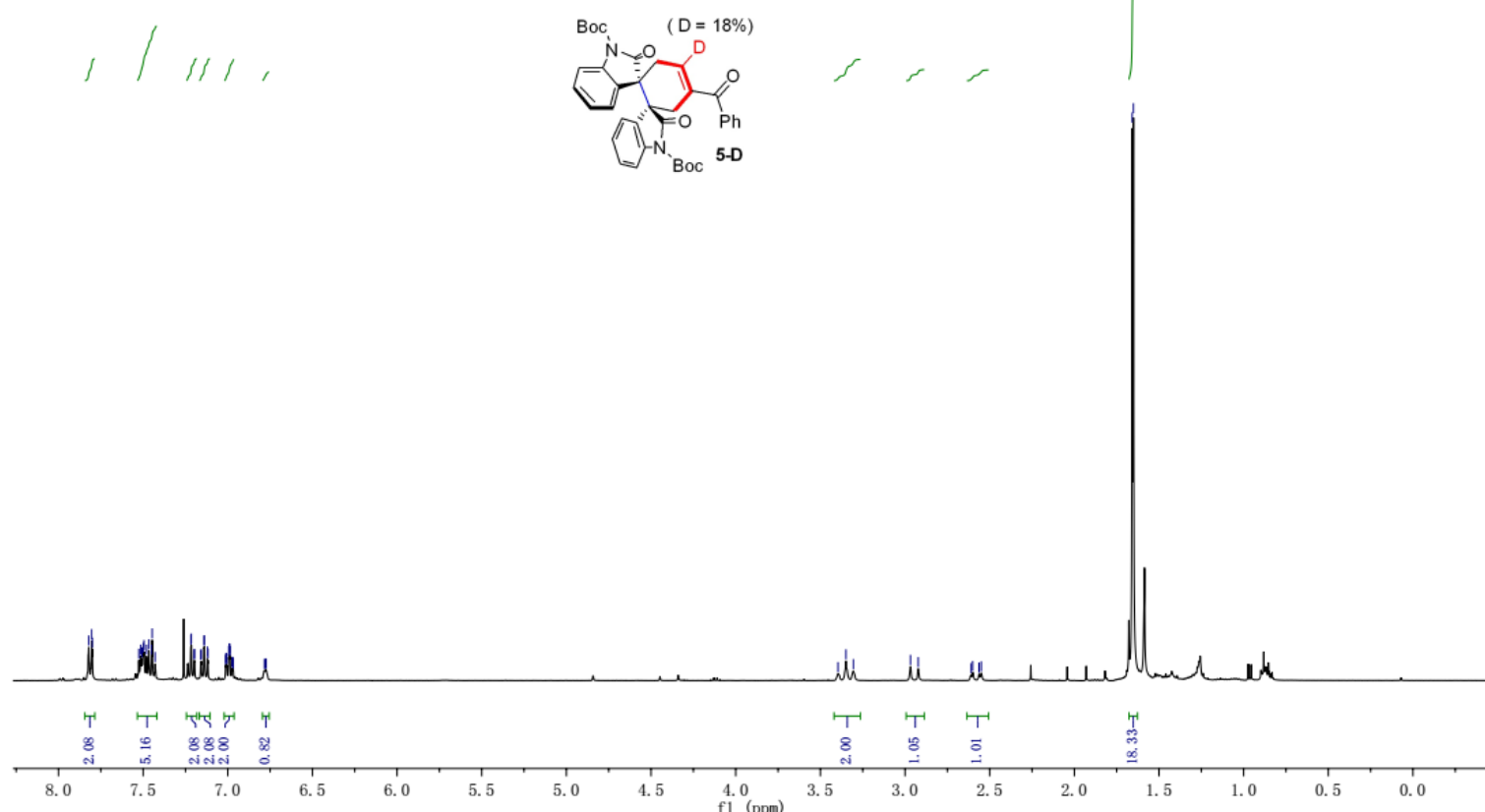


\section{Analytical Data and HPLC Chromatograms of the Products}

2-benzoylbuta-2,3-dien-1-yl acetate 1a<smiles>C=C=C(COC(C)=O)C(=O)c1ccccc1</smiles>

According to the allenic ketone preparation procedure, 1a was obtained as light-yellow oil (625 mg, 14.5\%yiled). ${ }^{1} \mathrm{H}$ NMR (500 MHz, $\left.\mathrm{CDCl}_{3}\right) \delta 7.83$ (d, $\left.J=7.3 \mathrm{~Hz}, 2 \mathrm{H}\right), 7.56$ $(\mathrm{dd}, J=10.9,3.9 \mathrm{~Hz}, 1 \mathrm{H}), 7.44(\mathrm{t}, J=7.7 \mathrm{~Hz}, 2 \mathrm{H}), 5.27(\mathrm{t}, J=2.2 \mathrm{~Hz}, 2 \mathrm{H}), 5.00(\mathrm{t}, J=$ $2.2 \mathrm{~Hz}, 2 \mathrm{H}), 2.10(\mathrm{~s}, 3 \mathrm{H}) .{ }^{13} \mathrm{C} \mathrm{NMR}\left(126 \mathrm{MHz}, \mathrm{CDCl}_{3}\right) \delta 216.5,192.4,170.5,137.5$, $132.5,129.1,128.0,103.1,80.9,61.0,20.8$. HRMS (ESI) $\mathrm{m} / \mathrm{z}$ calcd for $\mathrm{C}_{13} \mathrm{H}_{12} \mathrm{O}_{3}[\mathrm{M}+$ $\mathrm{Na}]^{+}=239.0679$, found: 239.0681 .

$\underline{\text { 2-(2-methylbenzoyl)buta-2,3-dien-1-yl acetate 1n }}$

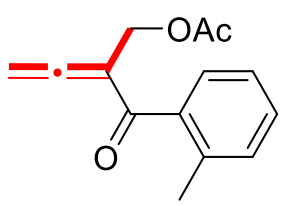

According to the allenic ketone preparation procedure, 1n was obtained as light-yellow oil (782 mg, 17\% yield). ${ }^{1} \mathrm{H}$ NMR $\left(500 \mathrm{MHz}, \mathrm{CDCl}_{3}\right) \delta 7.42-7.30(\mathrm{~m}, 2 \mathrm{H}), 7.24-7.13$ (m, 2H), 5.13 (t, $J=2.2 \mathrm{~Hz}, 2 \mathrm{H}), 4.98(\mathrm{t}, J=2.2 \mathrm{~Hz}, 2 \mathrm{H}), 2.38(\mathrm{~s}, 3 \mathrm{H}), 2.11(\mathrm{~s}, 3 \mathrm{H})$. ${ }^{13} \mathrm{C} \mathrm{NMR}\left(126 \mathrm{MHz}, \mathrm{CDCl}_{3}\right) \delta 217.4,195.8,170.5,138.4,136.2,130.8,130.3,128.1$, 124.7, 105.3, 80.7, 60.1, 20.8, 19.6. HRMS (ESI) m/z calcd for $\mathrm{C}_{14} \mathrm{H}_{14} \mathrm{O}_{3}[\mathrm{M}+\mathrm{Na}]^{+}=$ 253.0835, found: 253.0837 .

2-(3-methylbenzoyl)buta-2,3-dien-1-yl acetate 10<smiles>C=C=C(COC(C)=O)C(=O)c1cccc(C)c1</smiles>

According to the allenic ketone preparation procedure, 10 was obtained as light-yellow oil (759 mg, $16.5 \%$ yield). ${ }^{1} \mathrm{H} \mathrm{NMR}\left(500 \mathrm{MHz}, \mathrm{CDCl}_{3}\right) \delta 7.62(\mathrm{~d}, J=7.1 \mathrm{~Hz}, 2 \mathrm{H}), 7.41$ - 7.27 (m, 2H), $5.26(\mathrm{~s}, 2 \mathrm{H}), 5.00(\mathrm{~s}, 2 \mathrm{H}), 2.41$ (s, 3H), 2.10 (s, 3H). ${ }^{13} \mathrm{C}$ NMR (126 $\left.\mathrm{MHz}, \mathrm{CDCl}_{3}\right) \delta 216.5,192.6,170.6,137.9,137.5,133.2,129.5,127.8,126.3,103.1$, 80.8, 61.0, 21.3, 20.8. HRMS (ESI) $\mathrm{m} / \mathrm{z}$ calcd for $\mathrm{C}_{14} \mathrm{H}_{14} \mathrm{O}_{3}[\mathrm{M}+\mathrm{Na}]^{+}=253.0835$, found: 253.0836 .

2-(4-methylbenzoyl)buta-2,3-dien-1-yl acetate 1p<smiles>C=C=C(COC(C)=O)C(=O)c1ccc(C)cc1</smiles> 
According to the allenic ketone preparation procedure, 1p was obtained as light-yellow oil (874 mg, 19\% yield). ${ }^{1} \mathrm{H} \mathrm{NMR}\left(500 \mathrm{MHz}, \mathrm{CDCl}_{3}\right) \delta 7.75(\mathrm{~d}, J=8.2 \mathrm{~Hz}, 2 \mathrm{H}), 7.24$ $(\mathrm{d}, J=8.1 \mathrm{~Hz}, 2 \mathrm{H}), 5.26(\mathrm{t}, J=2.3 \mathrm{~Hz}, 2 \mathrm{H}), 4.99(\mathrm{t}, J=2.3 \mathrm{~Hz}, 2 \mathrm{H}), 2.42(\mathrm{~s}, 3 \mathrm{H}), 2.10$ $(\mathrm{s}, 3 \mathrm{H}) .{ }^{13} \mathrm{C} \mathrm{NMR}\left(126 \mathrm{MHz}, \mathrm{CDCl}_{3}\right) \delta 216.2,192.0,170.6,143.4,134.8,129.3,128.7$, 102.9, 80.8, 61.1, 21.6, 20.9. HRMS (ESI) $\mathrm{m} / \mathrm{z}$ calcd for $\mathrm{C}_{14} \mathrm{H}_{14} \mathrm{O}_{3}[\mathrm{M}+\mathrm{Na}]^{+}=$ 253.0835, found: 253.0838 .

2-(cyclohexanecarbonyl)buta-2,3-dien-1-yl acetate $\mathbf{1 q}$

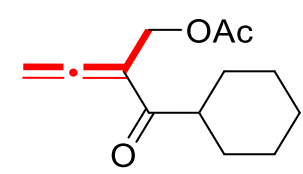

According to the allenic ketone preparation procedure, 1n was obtained as light-yellow oil (843 mg, 20\% yield). ${ }^{1} \mathrm{H}$ NMR (500 MHz, $\left.\mathrm{CDCl}_{3}\right) \delta 5.35$ (dd, $J=3.1,1.6 \mathrm{~Hz}, 2 \mathrm{H}$ ), $4.76(\mathrm{t}, J=2.4 \mathrm{~Hz}, 2 \mathrm{H}), 3.04-2.87(\mathrm{~m}, 1 \mathrm{H}), 2.10-1.99(\mathrm{~m}, 3 \mathrm{H}), 1.81-1.72(\mathrm{~m}, 4 \mathrm{H})$, 1.66 (ddd, $J=4.3,3.6,1.4 \mathrm{~Hz}, 1 \mathrm{H}), 1.37$ (dt, $J=12.1,7.2 \mathrm{~Hz}, 2 \mathrm{H}), 1.30-1.17$ (m, 3H). ${ }^{13} \mathrm{C} \mathrm{NMR}\left(126 \mathrm{MHz}, \mathrm{CDCl}_{3}\right) \delta 215.3,202.6,170.5,103.5,81.1,59.8,46.7,29.2,25.7$, 25.5, 20.8. HRMS (ESI) $\mathrm{m} / \mathrm{z}$ calcd for $\mathrm{C}_{13} \mathrm{H}_{18} \mathrm{O}_{3}[\mathrm{M}+\mathrm{Na}]^{+}=245.1148$, found: 245.1151 .

Ethyl 1,3-dibenzoylcyclopent-3-ene-1-carboxylate 3a<smiles>CCOC(=O)C1(C(=O)c2ccccc2)CC=C(C(=O)c2ccccc2)C1</smiles>

According to general procedure of $[4+1]$ annulation reaction, 3a was obtained in $85 \%$ yield $(29 \mathrm{mg})$ as colorless oil. ${ }^{1} \mathrm{H} \mathrm{NMR}\left(500 \mathrm{MHz}, \mathrm{CDCl}_{3}\right) \delta 7.89(\mathrm{~d}, J=8.3 \mathrm{~Hz}, 2 \mathrm{H})$, $7.72(\mathrm{~d}, J=8.1 \mathrm{~Hz}, 2 \mathrm{H}), 7.54(\mathrm{~m}, 2 \mathrm{H}), 7.44(\mathrm{dt}, J=12.1,7.7 \mathrm{~Hz}, 4 \mathrm{H}), 6.40(\mathrm{~s}, 1 \mathrm{H})$, $4.12(\mathrm{~m}, 2 \mathrm{H}), 3.67$ (d, $J=17.0 \mathrm{~Hz}, 2 \mathrm{H}), 3.56$ (d, $J=19.3 \mathrm{~Hz}, 1 \mathrm{H}), 3.28$ (d, J=22.6 Hz, 1H), 1.02 (t, $J=7.1 \mathrm{~Hz}, 3 \mathrm{H})$.

(1- nitrocyclopent-3-ene-1,3-diyl)bis(phenylmethanone) $\mathbf{3 b}$<smiles>O=C(C1=CCC(C(=O)c2ccccc2)([N+](=O)[O-])C1)c1ccccc1</smiles>

According to general procedure of $[4+1]$ annulation reaction, $\mathbf{3 b}$ was obtained in $87 \%$ yield (28 mg) as colorless oil. ${ }^{1} \mathrm{H}$ NMR $\left(400 \mathrm{MHz}, \mathrm{CDCl}_{3}\right) \delta 7.81(\mathrm{~s}, 1 \mathrm{H}), 7.76(\mathrm{~m}, 2 \mathrm{H})$, 
$7.62(\mathrm{~s}, 1 \mathrm{H}), 7.48(\mathrm{~m}, 1 \mathrm{H}), 6.46(\mathrm{~m}, 1 \mathrm{H}), 4.09(\mathrm{~m}, 1 \mathrm{H}), 4.04(\mathrm{~m}, 1 \mathrm{H}), 3.72(\mathrm{~s}, 1 \mathrm{H}), 3.60$ $(\mathrm{s}, 1 \mathrm{H})$.

Ethyl 3-benzoyl-1-cyanocyclopent-3-ene-1-carboxylate 3c<smiles>CCOC(=O)C1(C#N)CC=C(C(=O)c2ccccc2)C1</smiles>

According to general procedure of $[4+1]$ annulation reaction, $3 \mathbf{c}$ was obtained in $87 \%$ yield $(24 \mathrm{mg})$ as colorless oil. ${ }^{1} \mathrm{H}$ NMR $\left(500 \mathrm{MHz}, \mathrm{CDCl}_{3}\right) \delta 7.75(\mathrm{~m}, 2 \mathrm{H}), 7.57(\mathrm{~m}$, $1 \mathrm{H}), 7.46(\mathrm{t}, J=7.7 \mathrm{~Hz}, 2 \mathrm{H}), 6.43(\mathrm{~m}, 1 \mathrm{H}), 4.33(\mathrm{td}, J=7.5,0.7 \mathrm{~Hz}, 2 \mathrm{H}), 3.49(\mathrm{dd}, J=$ 6.1, 4.4 Hz, 2H), 3.45 (dd, $J=11.5,9.2 \mathrm{~Hz}, 1 \mathrm{H}), 3.31$ (dt, $J=19.0,2.0 \mathrm{~Hz}, 1 \mathrm{H}), 1.37$ (t, $J=7.1 \mathrm{~Hz}, 3 \mathrm{H})$.

3-benzoylcyclopent-3-ene-1,1-dicarbonitrile 3d<smiles>N#CC1(C#N)CC=C(C(=O)c2ccccc2)C1</smiles>

According to general procedure of $[4+1]$ annulation reaction, 3d was obtained in $99 \%$ yield $(22 \mathrm{mg})$ as colorless oil. ${ }^{1} \mathrm{H}$ NMR $\left(400 \mathrm{MHz}, \mathrm{CDCl}_{3}\right) \delta 7.69(\mathrm{~m}, 2 \mathrm{H}), 7.53(\mathrm{~m}$, 1H), $7.42(\mathrm{t}, J=7.6 \mathrm{~Hz}, 2 \mathrm{H}), 6.41(\mathrm{~m}, 1 \mathrm{H}), 3.56(\mathrm{dd}, J=3.6,1.8 \mathrm{~Hz}, 2 \mathrm{H}), 3.44(\mathrm{dd}, J$ $=4.3,2.0 \mathrm{~Hz}, 2 \mathrm{H})$.

\section{1,1'-(3-benzoylcyclopent-3-ene-1,1-diyl)bis(ethan-1-one) $\mathbf{3 e}$}<smiles>CC(=O)C1(C(C)=O)CC=C(C(=O)c2ccccc2)C1</smiles>

According to general procedure of $[4+1]$ annulation reaction, 3e was obtained in $98 \%$ yield $(25 \mathrm{mg})$ as colorless oil. ${ }^{1} \mathrm{H} \mathrm{NMR}\left(500 \mathrm{MHz}, \mathrm{CDCl}_{3}\right) \delta 7.71(\mathrm{dd}, J=5.2,3.2 \mathrm{~Hz}$, 2H), $7.59-7.53(\mathrm{~m}, 1 \mathrm{H}), 7.48-7.43(\mathrm{~m}, 2 \mathrm{H}), 6.37(\mathrm{dd}, J=3.1,1.2 \mathrm{~Hz}, 1 \mathrm{H}), 3.37(\mathrm{~d}$, $J=2.0 \mathrm{~Hz}, 2 \mathrm{H}), 3.23(\mathrm{~d}, J=2.3 \mathrm{~Hz}, 2 \mathrm{H}), 2.22(\mathrm{~s}, 6 \mathrm{H})$.

Ethyl 1-acetyl-3-benzoylcyclopent-3-ene-1-carboxylate $\mathbf{3 f}$ 
<smiles>CCOC(=O)C1(C(C)=O)CC=C(C(=O)c2ccccc2)C1</smiles>

According to general procedure of [ $4+1]$ annulation reaction, $3 \mathbf{f}$ was obtained in $96 \%$ yield $(27.4 \mathrm{mg})$ as colorless oil. ${ }^{1} \mathrm{H}$ NMR $\left(500 \mathrm{MHz}, \mathrm{CDCl}_{3}\right) \delta 7.77-7.69(\mathrm{~m}, 2 \mathrm{H})$, $7.55(\mathrm{~d}, J=7.4 \mathrm{~Hz}, 1 \mathrm{H}), 7.45(\mathrm{t}, J=7.7 \mathrm{~Hz}, 2 \mathrm{H}), 6.41-6.37(\mathrm{~m}, 1 \mathrm{H}), 4.28(\mathrm{qd}, J=7.1$, $1.9 \mathrm{~Hz}, 2 \mathrm{H}), 3.52-3.42(\mathrm{~m}, 1 \mathrm{H}), 3.39-3.26(\mathrm{~m}, 2 \mathrm{H}), 3.23-3.11(\mathrm{~m}, 1 \mathrm{H}), 2.27(\mathrm{~s}$, $3 \mathrm{H}), 1.32(\mathrm{t}, J=7.1 \mathrm{~Hz}, 3 \mathrm{H})$.

Di-tert-butyl (2'S,3S)-4'-benzoyl-2,2"-dioxodispiro[indoline-3,1'-cyclohexane-2',3"indolin]-4'-ene-1,1"-dicarboxylate 5a<smiles>CC(C)(C)OC(=O)c1ccccc1C1(c2ccccc2)CCC(C(=O)c2ccccc2)(c2ccccc2)C(=O)N1c1ccccc1</smiles>

According to general [4 +2$]$ annulation procedure, 5a was obtained in 98\% yield $(60.7$ $\mathrm{mg}$ ) as an orange solid, $95 \% e e .[\alpha]_{\mathrm{D}}^{25}=-171.4\left(\mathrm{c} 1.0, \mathrm{CHCl}_{3}\right)$. The ee was determined by chiral stationary phase HPLC analysis [Daicel Chiralpak IA, isopropanol/hexane = $5 / 95,0.8 \mathrm{~mL} / \mathrm{min}, \lambda=254 \mathrm{~nm}, \mathrm{t}_{\mathrm{R}}$ (major) $=12.2 \mathrm{~min}, \mathrm{t}_{\mathrm{R}}($ minor $\left.)=11.1 \mathrm{~min}\right] .{ }^{1} \mathrm{H} \mathrm{NMR}$ $\left(500 \mathrm{MHz}, \mathrm{CDCl}_{3}\right) \delta 7.87-7.80(\mathrm{~m}, 2 \mathrm{H}), 7.58-7.45(\mathrm{~m}, 5 \mathrm{H}), 7.28-7.22(\mathrm{~m}, 2 \mathrm{H})$, $7.20-7.14(\mathrm{~m}, 2 \mathrm{H}), 7.06-6.99(\mathrm{~m}, 2 \mathrm{H}), 6.81(\mathrm{~d}, J=3.5 \mathrm{~Hz}, 1 \mathrm{H}), 3.38(\mathrm{t}, J=19.8 \mathrm{~Hz}$, 2H), 2.97 (d, $J=18.2 \mathrm{~Hz}, 1 \mathrm{H}), 2.61(\mathrm{dd}, J=19.8,4.4 \mathrm{~Hz}, 1 \mathrm{H}), 1.68$ (d, $J=3.6 \mathrm{~Hz}$, $18 \mathrm{H}) .{ }^{13} \mathrm{C} \mathrm{NMR}\left(126 \mathrm{MHz}, \mathrm{CDCl}_{3}\right) \delta 196.8,175.0,174.9,148.6,148.6,139.5,139.2$, 139.0, 138.4, 135.3, 131.6, 129.5, 129.3, 129.2, 128.1, 127.2, 126.9, 124.1, 124.1, 122.9, 122.8, 114.5, 84.7, 84.6, 50.1, 49.7, 31.9, 29.6, 28.1. HRMS (ESI) m/z calcd for $\mathrm{C}_{33} \mathrm{H}_{27} \mathrm{NO}_{4} \mathrm{~S}[\mathrm{M}+\mathrm{Na}]^{+}=643.2415$, found: 643.2420 .

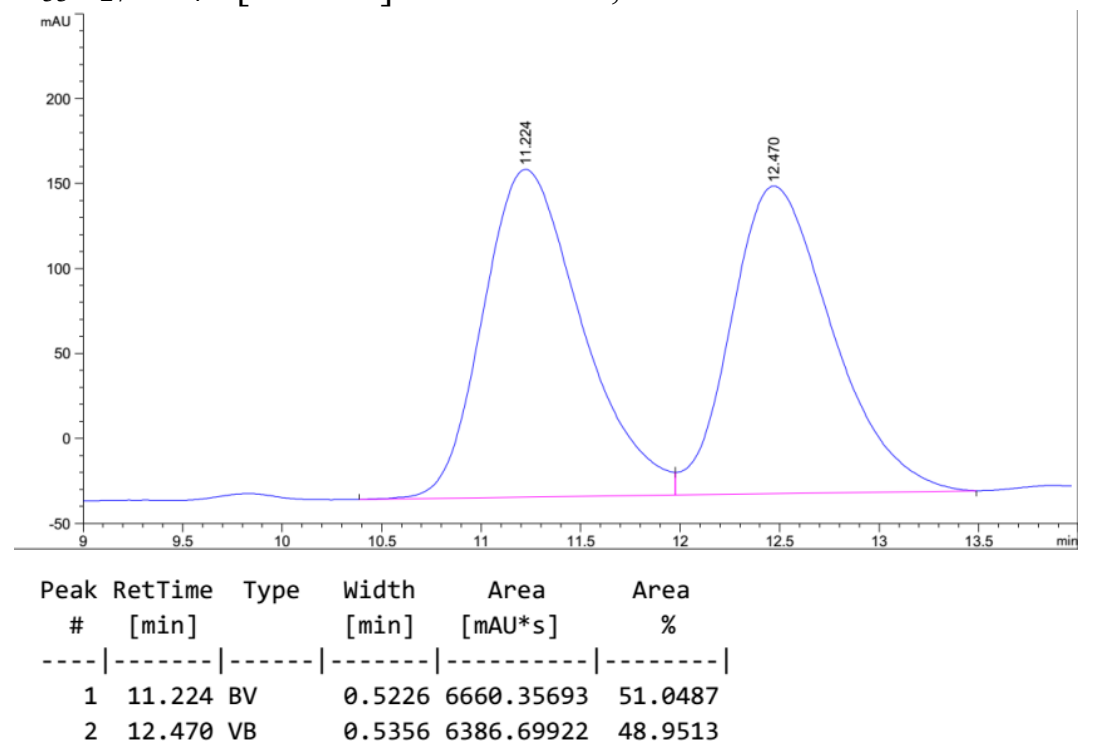




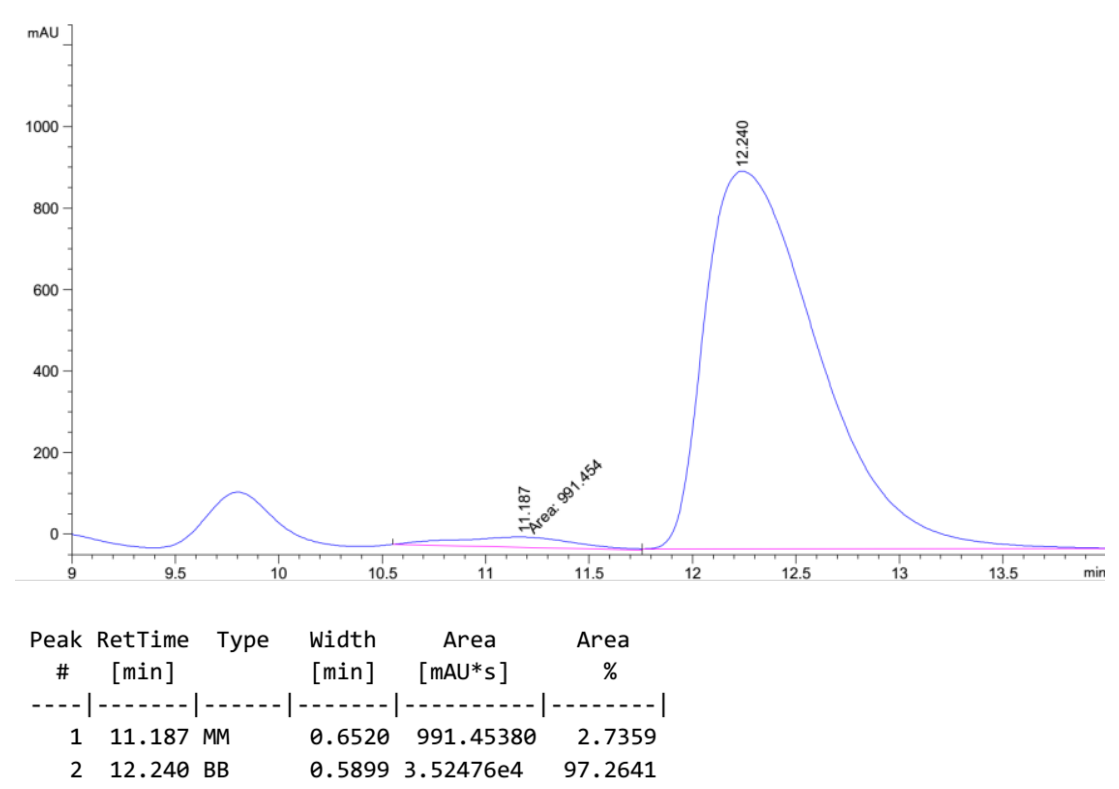

Di-tert-butyl (2'S,3S)-4'-benzoyl-5,5"-difluoro-2,2"-dioxodispiro[indoline-3,1'cyclohexane-2',3"-indolin]-4'-ene-1,1"-dicarboxylate $\mathbf{5 b}$

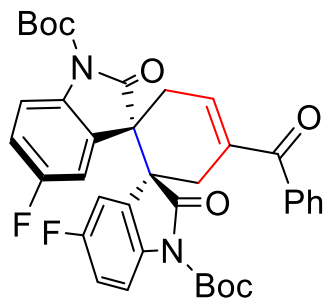

According to general [ $4+2]$ annulation procedure, $\mathbf{5 b}$ was obtained in $94 \%$ yield $(61.7$ $\mathrm{mg}$ ) as a deep red solid, $87 \%$ ee. $[\alpha]_{\mathrm{D}}^{25}=-84.7\left(\mathrm{c} 1.0, \mathrm{CHCl}_{3}\right)$. The ee was determined by chiral stationary phase HPLC analysis [Daicel Chiralpak IA, isopropanol/hexane = $5 / 95,0.8 \mathrm{~mL} / \mathrm{min}, \lambda=254 \mathrm{~nm}, \mathrm{t}_{\mathrm{R}}$ (major) $=10.4 \mathrm{~min}, \mathrm{t}_{\mathrm{R}}($ minor $\left.)=9.6 \mathrm{~min}\right] .{ }^{1} \mathrm{H} \mathrm{NMR}$ $\left(500 \mathrm{MHz}, \mathrm{CDCl}_{3}\right) \delta 7.87-7.79(\mathrm{~m}, 2 \mathrm{H}), 7.58(\mathrm{ddd}, J=19.8,9.8,4.7 \mathrm{~Hz}, 3 \mathrm{H}), 7.47$ $(\mathrm{dd}, J=10.9,3.9 \mathrm{~Hz}, 2 \mathrm{H}), 7.04-6.95(\mathrm{~m}, 2 \mathrm{H}), 6.90(\mathrm{dd}, J=8.6,7.2 \mathrm{~Hz}, 2 \mathrm{H}), 6.79(\mathrm{~s}$, $1 \mathrm{H}), 3.31(\mathrm{t}, J=20.0 \mathrm{~Hz}, 2 \mathrm{H}), 2.99(\mathrm{~d}, J=18.0 \mathrm{~Hz}, 1 \mathrm{H}), 2.62(\mathrm{dd}, J=19.8,4.6 \mathrm{~Hz}$, $1 \mathrm{H}), 1.68(\mathrm{~d}, J=3.3 \mathrm{~Hz}, 18 \mathrm{H}) .{ }^{13} \mathrm{C} \mathrm{NMR}\left(126 \mathrm{MHz}, \mathrm{CDCl}_{3}\right) \delta 196.5,174.3,160.6$, $158.7,148.5,148.5,138.9,138.2$, 135.1, 135.0, 134.9, 131.7, 129.5, 128.1, 116.2, 116.1, 116.0, 110.8, 110.7, 110.6, 110.5, 85.3, 85.2, 50.2, 49.7, 31.8, 29.5, 28.0. HRMS (ESI) $\mathrm{m} / \mathrm{z}$ calcd for $\mathrm{C}_{37} \mathrm{H}_{34} \mathrm{~F}_{2} \mathrm{~N}_{2} \mathrm{O}_{7}[\mathrm{M}+\mathrm{Na}]^{+}=679.2226$, found: 679.2229 . 

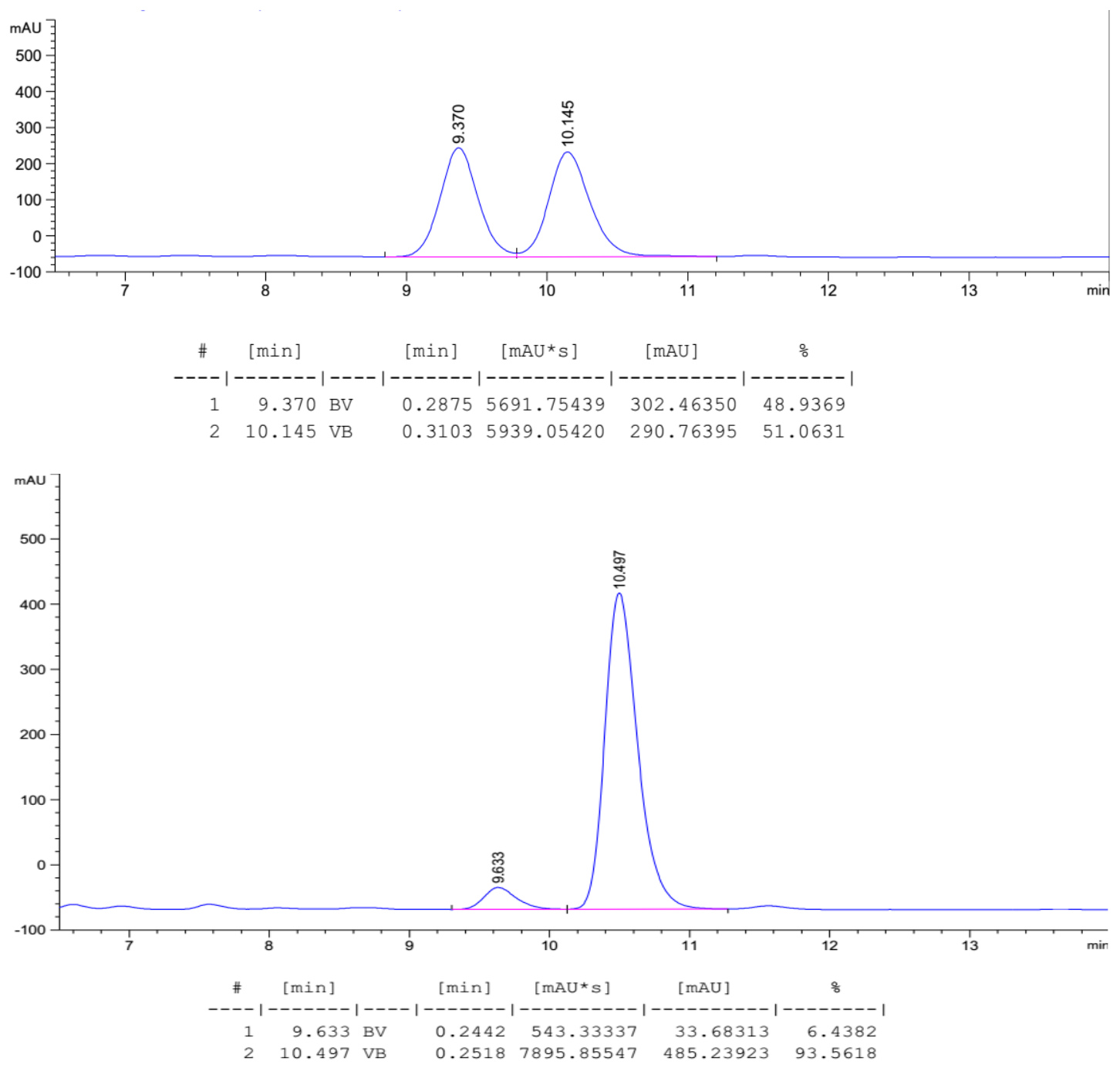

Di-tert-butyl (2'S,3S)-4'-benzoyl-5,5"-dichloro-2,2"-dioxodispiro[indoline-3,1'cyclohexane-2',3"-indolin]-4'-ene-1,1"-dicarboxylate 5c<smiles>CC(C)(C)OC(=O)N1C=CC(C(=O)c2ccccc2)=CC[C@@]2(C(=O)O)c3cc(Cl)ccc3N(C(=O)OC(C)(C)C)c3ccc(Cl)cc3C2(c2ccccc2)OC1=O</smiles>

According to general [4+2] annulation procedure, $\mathbf{5 c}$ was obtained in $96 \%$ yield (66.2 $\mathrm{mg})$ as a deep red solid and $98 \% e e .[\alpha]_{\mathrm{D}}^{25}=-128.7\left(\mathrm{c} 1.0, \mathrm{CHCl}_{3}\right)$. The ee was determined by chiral stationary phase HPLC analysis [Daicel Chiralpak IA, isopropanol $/$ hexane $=5 / 95,0.8 \mathrm{~mL} / \mathrm{min}, \lambda=254 \mathrm{~nm}, \mathrm{t}_{\mathrm{R}}$ (major) $=11.5 \mathrm{~min}, \mathrm{t}_{\mathrm{R}}$ (minor) $=9.0 \mathrm{~min}] .{ }^{1} \mathrm{H}$ NMR $\left(500 \mathrm{MHz}, \mathrm{CDCl}_{3}\right) \delta 7.82(\mathrm{~d}, J=7.6 \mathrm{~Hz}, 2 \mathrm{H}), 7.56(\mathrm{~d}, J=8.7 \mathrm{~Hz}$, $3 \mathrm{H}), 7.48(\mathrm{~d}, J=7.1 \mathrm{~Hz}, 2 \mathrm{H}), 7.22(\mathrm{~d}, J=12.3 \mathrm{~Hz}, 2 \mathrm{H}), 7.17(\mathrm{dd}, J=8.6,1.4 \mathrm{~Hz}, 2 \mathrm{H})$, $6.78(\mathrm{~s}, 1 \mathrm{H}), 3.31(\mathrm{t}, J=18.9 \mathrm{~Hz}, 2 \mathrm{H}), 2.97(\mathrm{~d}, J=18.2 \mathrm{~Hz}, 1 \mathrm{H}), 2.60(\mathrm{dd}, J=19.6,4.7$ $\mathrm{Hz}, 1 \mathrm{H}), 1.69(\mathrm{~d}, J=3.1 \mathrm{~Hz}, 18 \mathrm{H}) .{ }^{13} \mathrm{C}$ NMR $\left(126 \mathrm{MHz}, \mathrm{CDCl}_{3}\right) \delta 196.5,173.9,148.4$, 
$148.3,138.8,138.1,137.7,137.5,135.0,131.7,129.9,129.5,129.5,128.7,128.5,128.1$, 123.4, 123.2, 115.8, 85.6, 85.4, 50.0, 49.5, 31.8, 29.6, 28.0. HRMS (ESI) m/z calcd for $\mathrm{C}_{37} \mathrm{H}_{34} \mathrm{Cl}_{2} \mathrm{~N}_{2} \mathrm{O}_{7}[\mathrm{M}+\mathrm{Na}]^{+}=711.1635$, found: 711.1637.
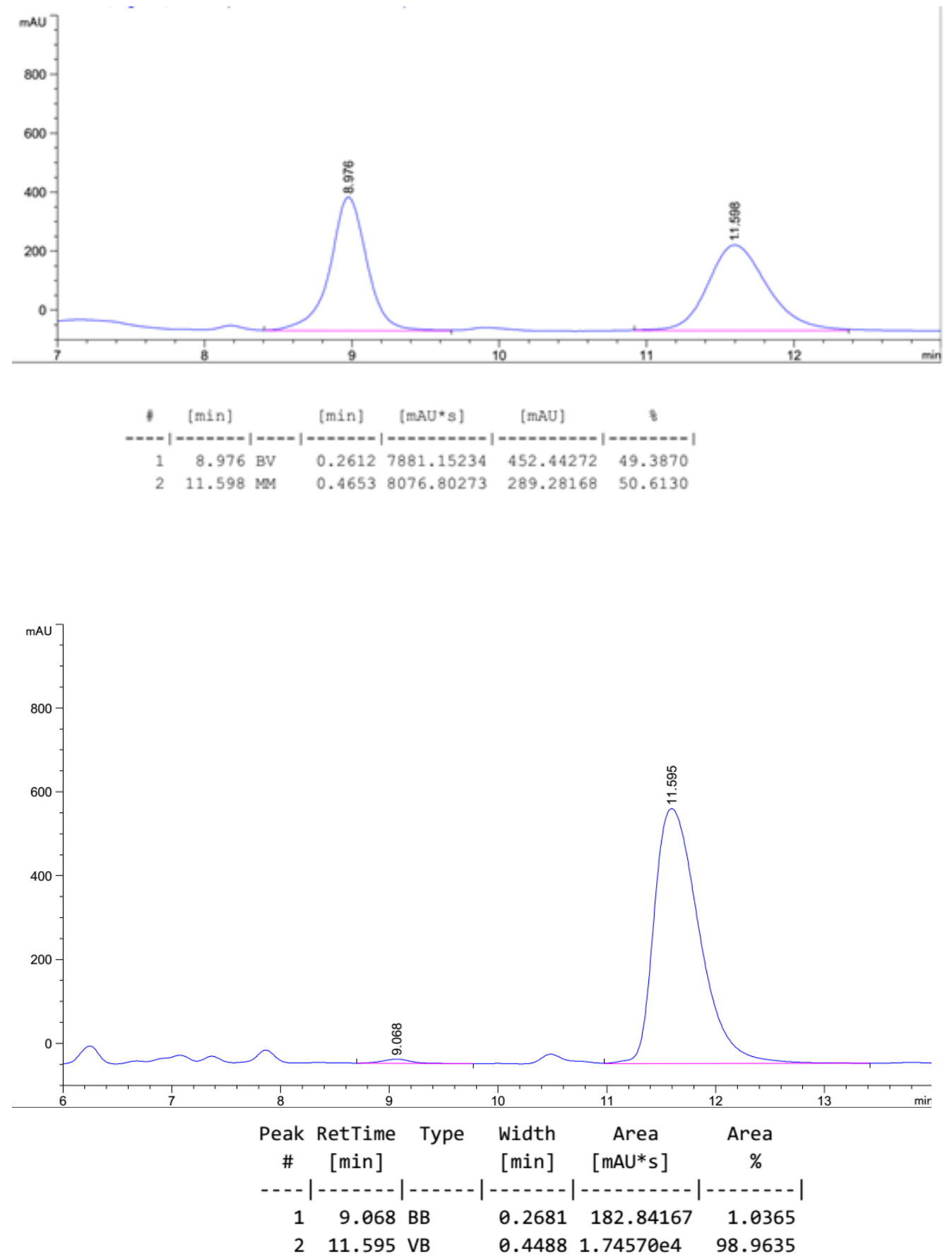
Di-tert-butyl (2'S,3S)-4'-benzoyl-5,5"-dibromo-2,2"-dioxodispiro[indoline-3,1'cyclohexane-2',3"-indolin]-4'-ene-1,1"-dicarboxylate 5d<smiles>CC(C)(C)OC(=O)N1C(=O)N(c2ccc(Br)cc2)C(=O)[C@@](C(=O)C(=O)c2ccccc2)(c2cc(Br)ccc2N(C(=O)OC(C)(C)C)C(=O)c2ccccc2)CC=C1C(=O)c1ccccc1</smiles>

According to general [4 +2] annulation procedure, 5d was obtained in 95\% yield (73.8 $\mathrm{mg})$ as a dark red solid and $96 \% e e .[\alpha]_{\mathrm{D}}^{25}=-91.4\left(\mathrm{c} 1.0, \mathrm{CHCl}_{3}\right)$. The $e e$ was determined by chiral stationary phase HPLC analysis [Daicel Chiralpak IA, isopropanol/hexane $=$ $5 / 95,0.8 \mathrm{~mL} / \mathrm{min}, \lambda=254 \mathrm{~nm}, \mathrm{t}_{\mathrm{R}}$ (major) $=11.6 \mathrm{~min}, \mathrm{t}_{\mathrm{R}}($ minor $\left.)=10.8 \mathrm{~min}\right] .{ }^{1} \mathrm{H} \mathrm{NMR}$ $\left(500 \mathrm{MHz}, \mathrm{CDCl}_{3}\right) \delta 7.84(\mathrm{~s}, 2 \mathrm{H}), 7.58-7.46(\mathrm{~m}, 5 \mathrm{H}), 7.25(\mathrm{~d}, J=6.7 \mathrm{~Hz}, 1 \mathrm{H}), 7.18$ (s, 1H), $7.06-6.99(\mathrm{~m}, 2 \mathrm{H}), 6.81(\mathrm{~s}, 1 \mathrm{H}), 3.38(\mathrm{t}, J=20.1 \mathrm{~Hz}, 2 \mathrm{H}), 2.97(\mathrm{~d}, J=18.4$ $\mathrm{Hz}, 1 \mathrm{H}), 2.61(\mathrm{~d}, J=19.2 \mathrm{~Hz}, 1 \mathrm{H}), 1.68(\mathrm{dd}, J=7.3,3.4 \mathrm{~Hz}, 18 \mathrm{H}) .{ }^{13} \mathrm{C} \mathrm{NMR}(126 \mathrm{MHz}$, $\left.\mathrm{CDCl}_{3}\right) \delta 196.8,175.0,174.9,148.6,139.5,139.2,139.0,138.4,135.3,131.6,129.5$, 129.3, 129.2, 128.1, 127.2, 127.0, 124.1, 122.9, 122.8, 114.5, 84.7, 84.6, 50.1, 49.7, 31.9, 29.7, 28.1.HRMS (ESI) $\mathrm{m} / \mathrm{z}$ calcd. for $\mathrm{C}_{37} \mathrm{H}_{34} \mathrm{Br}_{2} \mathrm{~N}_{2} \mathrm{O}_{7}[\mathrm{M}+\mathrm{Na}]^{+}=801.0605$, found: 801.0612 .

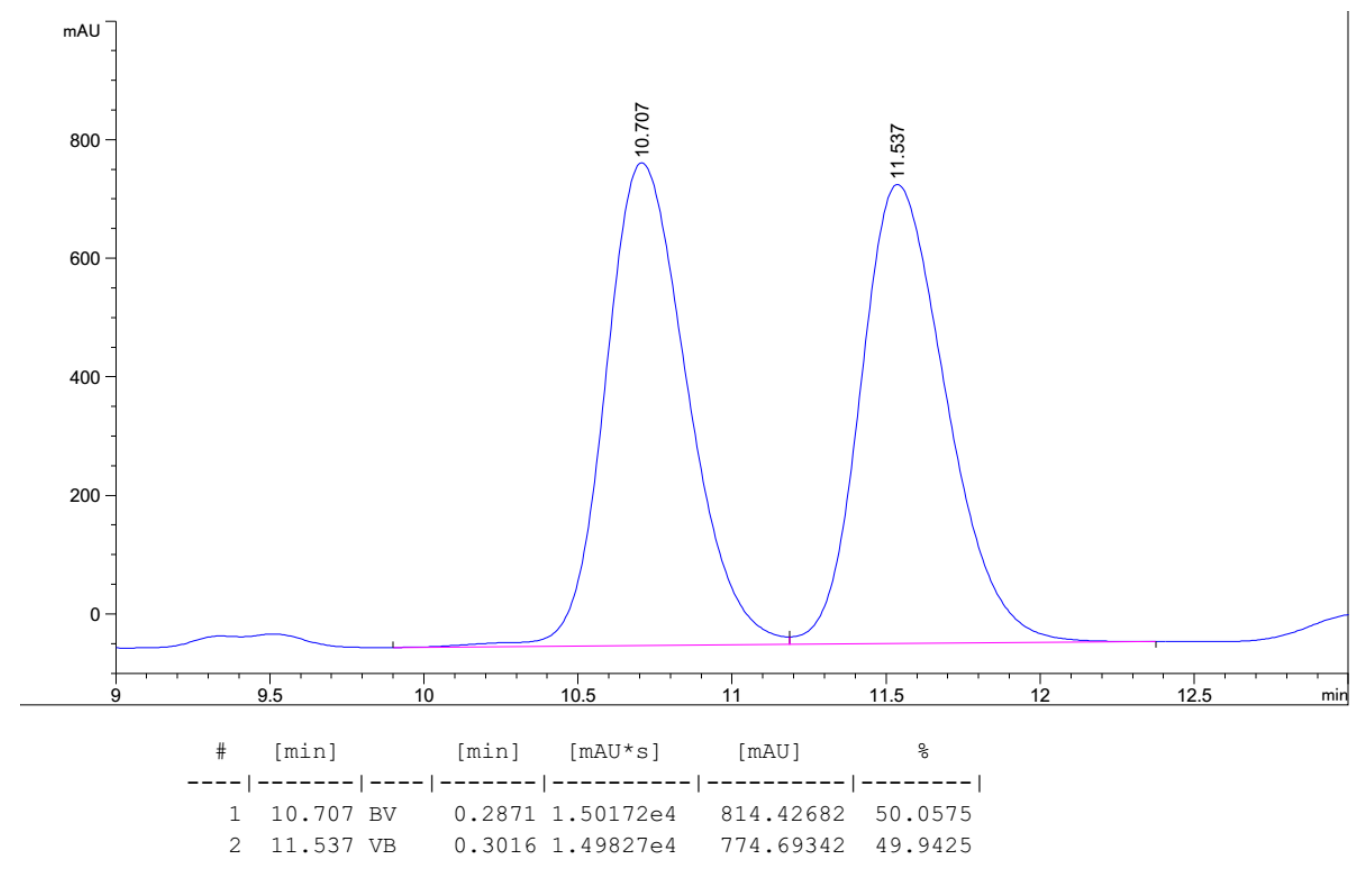




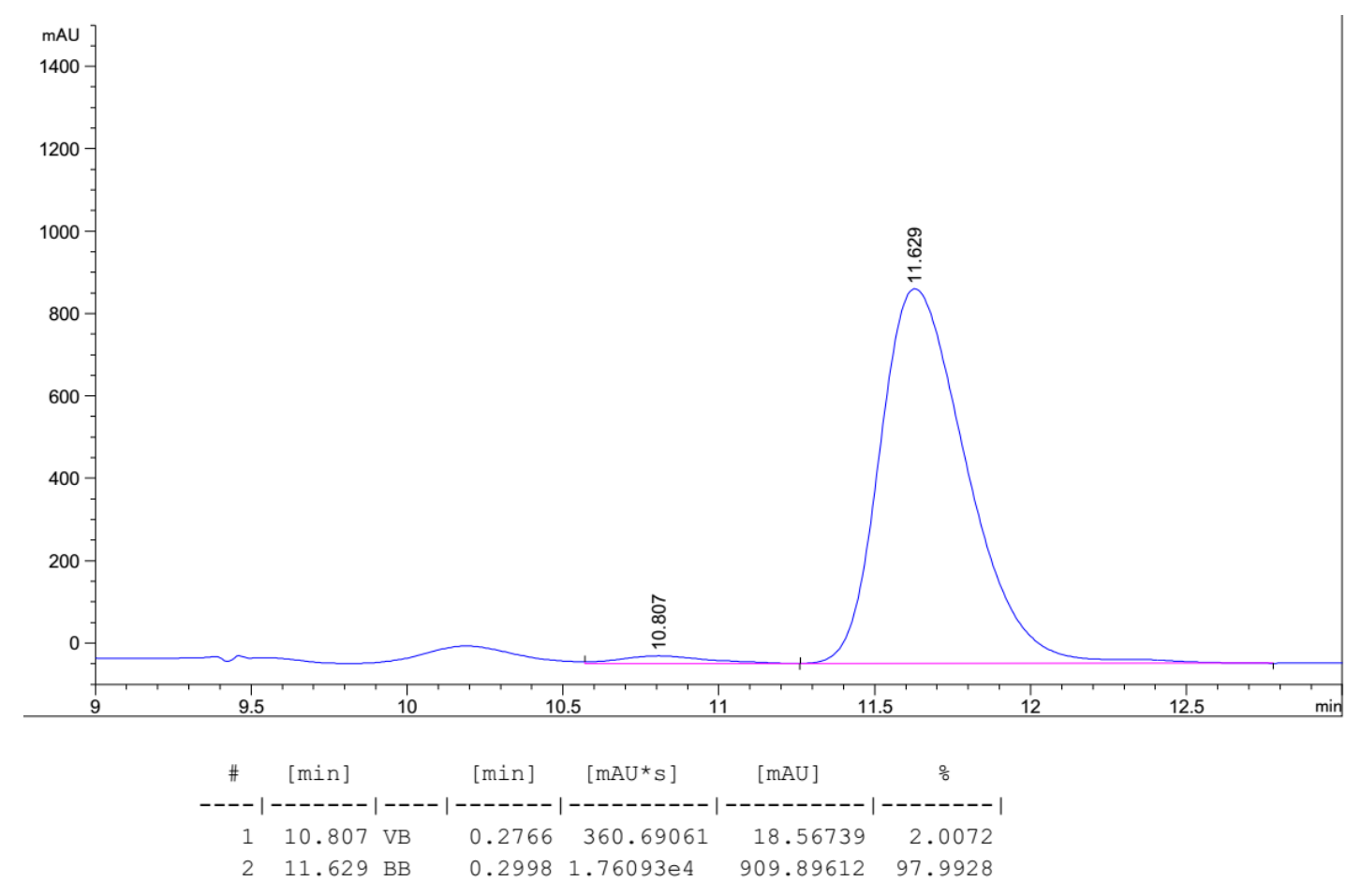

Di-tert-butyl (2'S,3S)-4'-benzoyl-5,5"-dimethyl-2,2"-dioxodispiro[indoline-3,1'cyclohexane-2',3"-indolin]-4'-ene-1,1"-dicarboxylate 5e<smiles>Cc1ccc(N(C(=O)OCc2ccccc2)C(=O)c2ccccc2)c(C2(C(=O)c3ccccc3)CC=C(C(=O)c3ccccc3)CC2)c1</smiles>

According to general $[4+2]$ annulation procedure, 5e was obtained in 93\% yield $(60.3$ $\mathrm{mg}$ ) as a deep red solid and $94 \% e e .[\alpha]_{\mathrm{D}}^{25}=-84.2\left(\mathrm{c} 1.0, \mathrm{CHCl}_{3}\right)$. The ee was determined by chiral stationary phase HPLC analysis [Daicel Chiralpak IA, isopropanol/hexane = $5 / 95,0.8 \mathrm{~mL} / \mathrm{min}, \lambda=254 \mathrm{~nm}, \mathrm{t}_{\mathrm{R}}$ (major) $=10 \mathrm{~min}, \mathrm{t}_{\mathrm{R}}($ minor $\left.)=11.5 \mathrm{~min}\right] .{ }^{1} \mathrm{H} \mathrm{NMR}$ $\left(500 \mathrm{MHz}, \mathrm{CDCl}_{3}\right) \delta 7.87-7.81(\mathrm{~m}, 2 \mathrm{H}), 7.55(\mathrm{t}, J=7.4 \mathrm{~Hz}, 1 \mathrm{H}), 7.47(\mathrm{t}, J=7.5 \mathrm{~Hz}$, 2H), $7.39(\mathrm{t}, J=7.8 \mathrm{~Hz}, 2 \mathrm{H}), 7.07(\mathrm{~d}, J=8.0 \mathrm{~Hz}, 2 \mathrm{H}), 6.96(\mathrm{~d}, J=8.3 \mathrm{~Hz}, 2 \mathrm{H}), 6.81-$ $6.77(\mathrm{~m}, 1 \mathrm{H}), 3.36(\mathrm{t}, J=19.1 \mathrm{~Hz}, 2 \mathrm{H}), 2.93(\mathrm{~d}, J=18.0 \mathrm{~Hz}, 1 \mathrm{H}), 2.58(\mathrm{dd}, J=19.6$, $4.5 \mathrm{~Hz}, 1 \mathrm{H}), 2.26(\mathrm{~d}, J=4.9 \mathrm{~Hz}, 6 \mathrm{H}), 1.68(\mathrm{~d}, J=2.6 \mathrm{~Hz}, 18 \mathrm{H}) .{ }^{13} \mathrm{C} \mathrm{NMR}(126 \mathrm{MHz}$, $\left.\mathrm{CDCl}_{3}\right) \delta 196.9,175.1,148.7,139.6,138.5,136.7,136.5,135.4,133.8,131.5,129.6$, 129.6, 128.0, 127.3, 127.1, 123.6, 123.5, 114.0, 84.5, 84.4, 50.0, 49.6, 32.1, 29.9, 28.1, 21.0, 21.0. HRMS (ESI) $\mathrm{m} / \mathrm{z}$ calcd for $\mathrm{C}_{39} \mathrm{H}_{40} \mathrm{~N}_{2} \mathrm{O}_{7}[\mathrm{M}+\mathrm{Na}]^{+}=671.2728$, found: 671.2735 . 

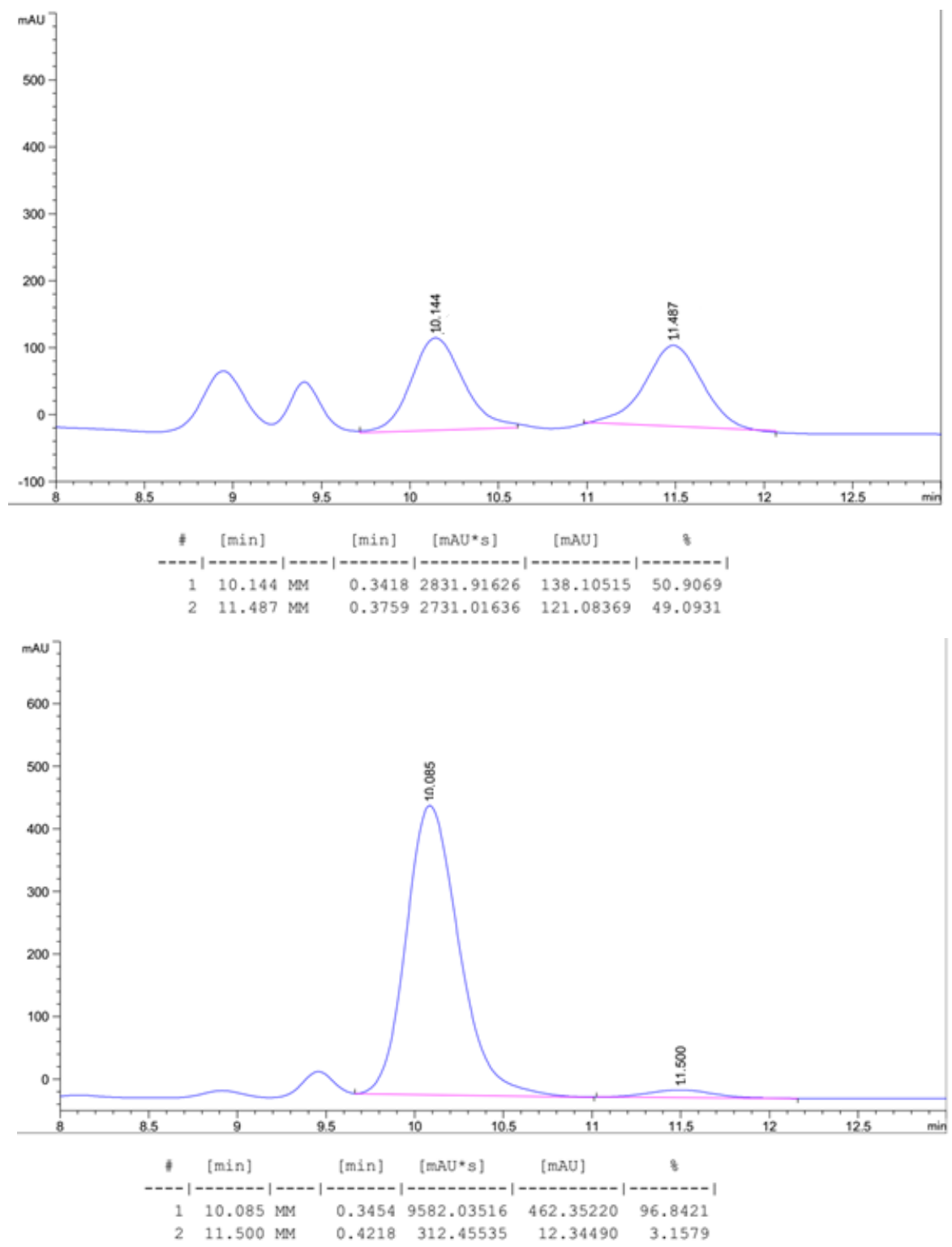

Di-tert-butyl (2'S,3S)-4'-benzoyl-6,6"-dibromo-2,2"-dioxodispiro[indoline-3,1'cyclohexane-2',3"-indolin]-4'-ene-1,1"-dicarboxylate $\mathbf{5 f}$<smiles>CC(C)(C)OC(=O)N(C(=O)c1ccccc1)c1cc(Br)ccc1C12ON(C(=O)OC(C)(C)C)C1(c1ccc(Br)cc1)CC=C(C(=O)c1ccccc1)C2=O</smiles>

According to general [ $4+2]$ annulation procedure, $\mathbf{5 f}$ was obtained in 94\% yield $(73.1$ $\mathrm{mg}$ ) as a deep red solid and $91 \% e e .[\alpha]_{\mathrm{D}}^{25}=-71.2\left(\mathrm{c} 1.0, \mathrm{CHCl}_{3}\right)$. The ee was determined by chiral stationary phase HPLC analysis [Daicel Chiralpak IA, isopropanol/hexane $=$ $5 / 95,0.8 \mathrm{~mL} / \mathrm{min}, \lambda=254 \mathrm{~nm}, \mathrm{t}_{\mathrm{R}}$ (major) $=13.6 \mathrm{~min}, \mathrm{t}_{\mathrm{R}}($ minor $\left.)=14.8 \mathrm{~min}\right] .{ }^{1} \mathrm{H} \mathrm{NMR}$ $\left(500 \mathrm{MHz}, \mathrm{CDCl}_{3}\right) \delta 7.86-7.80(\mathrm{~m}, 3 \mathrm{H}), 7.60-7.53(\mathrm{~m}, 2 \mathrm{H}), 7.51-7.43(\mathrm{~m}, 2 \mathrm{H})$, $7.18(\mathrm{~m}, J=8.0,3.5,1.7 \mathrm{~Hz}, 2 \mathrm{H}), 7.07(\mathrm{dd}, J=9.5,8.4 \mathrm{~Hz}, 2 \mathrm{H}), 6.77(\mathrm{~d}, J=3.1 \mathrm{~Hz}$, 
$1 \mathrm{H}), 3.32(\mathrm{t}, J=20.3 \mathrm{~Hz}, 2 \mathrm{H}), 2.94(\mathrm{~d}, J=18.2 \mathrm{~Hz}, 1 \mathrm{H}), 2.63-2.53(\mathrm{~m}, 1 \mathrm{H}), 1.68(\mathrm{~d}$, $J=2.4 \mathrm{~Hz}, 18 \mathrm{H}) .{ }^{13} \mathrm{C} \mathrm{NMR}\left(126 \mathrm{MHz}, \mathrm{CDCl}_{3}\right) \delta 196.5,174.2,148.2,140.2,140.0$, $138.8,138.1,135.0,131.7,129.5,128.1,127.4,127.3,126.1,125.9,124.1,124.0,123.3$, 118.3, 85.5, 85.5, 49.7, 49.2, 32.1, 29.9, 29.7, 28.1. HRMS (ESI) m/z calcd for $\mathrm{C}_{37} \mathrm{H}_{34} \mathrm{Br}_{2} \mathrm{~N}_{2} \mathrm{O}_{7}[\mathrm{M}+\mathrm{Na}]^{+}=801.0605$, found: 801.0611 .
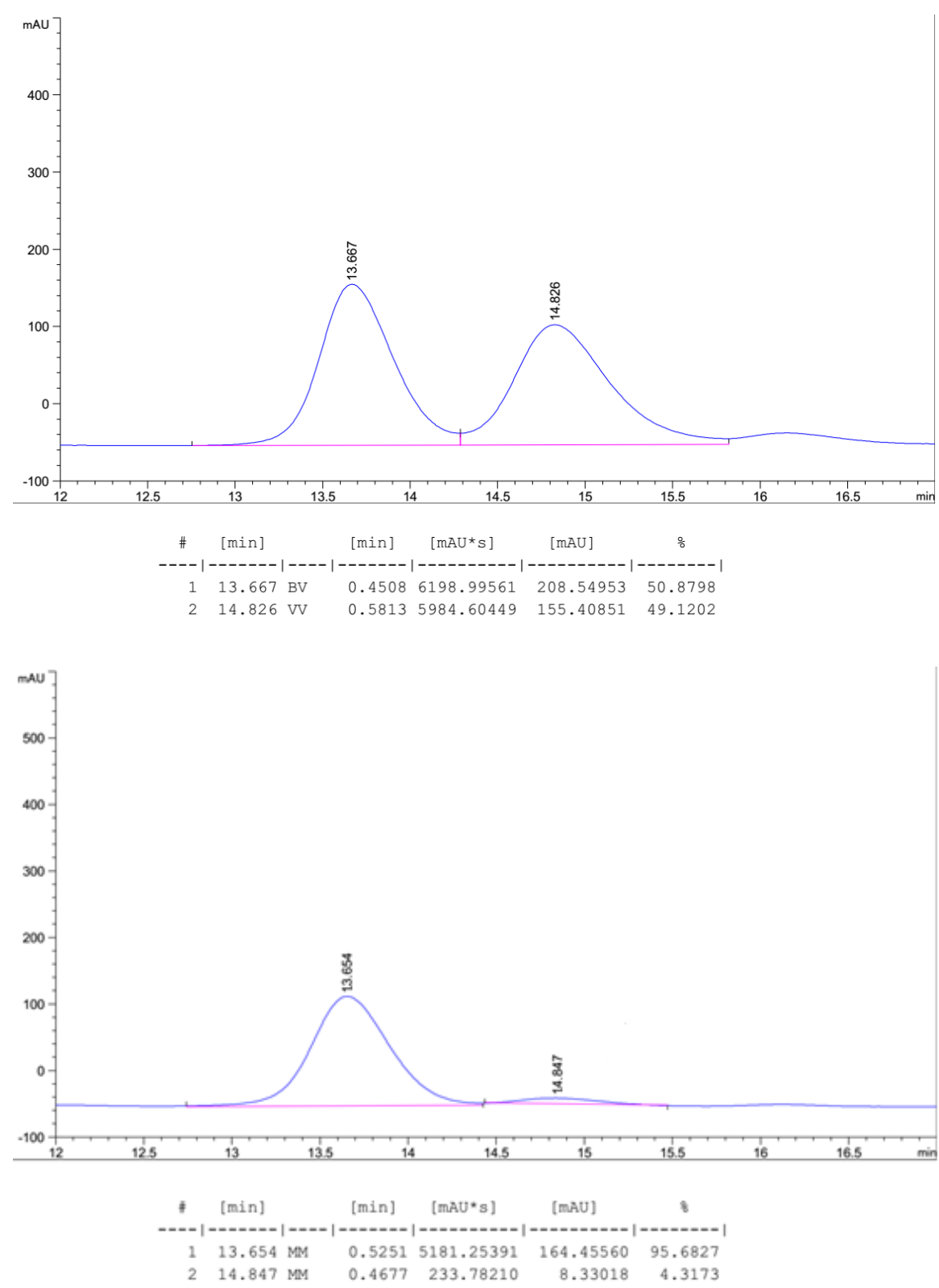

Di-tert-butyl (2'S,3S)-4'-benzoyl-5"-fluoro-2,2"-dioxodispiro[indoline-3,1'cyclohexane-2',3"-indolin]-4'-ene-1,1"-dicarboxylate $\mathbf{5 g}$ 


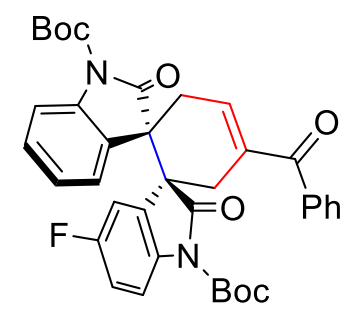

According to general [4 4 2] annulation procedure, 5g was obtained in $91 \%$ yield (58.1 $\mathrm{mg})$ as a deep red solid and 93\% ee. $\mathrm{rr}=4: 1$ determined by ${ }^{1} \mathrm{H}$ NMR. $[\alpha]_{\mathrm{D}}^{25}=-212.5(\mathrm{c}$ $1.0, \mathrm{CHCl}_{3}$ ). The ee was determined by chiral stationary phase HPLC analysis [Daicel Chiralpak IA, isopropanol $/$ hexane $=5 / 95,0.8 \mathrm{~mL} / \mathrm{min}, \lambda=254 \mathrm{~nm}, \mathrm{t}_{\mathrm{R}}$ (major) $=11.1$ min, $\mathrm{t}_{\mathrm{R}}($ minor $\left.)=9.5 \mathrm{~min}\right] .{ }^{1} \mathrm{H} \mathrm{NMR}\left(500 \mathrm{MHz}, \mathrm{CDCl}_{3}\right) \delta 7.83(\mathrm{dd}, J=8.2,1.2 \mathrm{~Hz}$, 2H), $7.60-7.47$ (m, 5H), 7.20 (m, J = 12.8, 6.8, $1.2 \mathrm{~Hz}, 2 \mathrm{H}), 7.06-7.00(\mathrm{~m}, 2 \mathrm{H}), 6.90$ $-6.85(\mathrm{~m}, 1 \mathrm{H}), 6.84-6.78(\mathrm{~m}, 1 \mathrm{H}), 3.34(\mathrm{dd}, J=40.2,21.5 \mathrm{~Hz}, 2 \mathrm{H}), 3.08(\mathrm{~d}, J=18.5$ $\mathrm{Hz}, 0.22 \mathrm{H}), 2.99(\mathrm{~d}, J=18.5 \mathrm{~Hz}, 0.88 \mathrm{H}), 2.66-2.58(\mathrm{~m}, 1 \mathrm{H}), 1.68(\mathrm{~d}, J=10.0 \mathrm{~Hz}$, 18H). ${ }^{13} \mathrm{C}$ NMR (126 MHz, $\left.\mathrm{CDCl}_{3}\right) \delta 196.8,175.0,174.9,148.6,148.6,139.5,139.2$, 139.0, 138.4, 135.3, 131.6, 129.5, 129.3, 129.2, 128.1, 127.2, 126.9, 124.1, 124.1, 122.9, 122.8, 114.5, 84.7, 84.6, 50.1, 49.7, 31.9, 29.6, 28.1. HRMS (ESI) $\mathrm{m} / \mathrm{z}$ calcd for $\mathrm{C}_{37} \mathrm{H}_{35} \mathrm{FN}_{2} \mathrm{O}_{7}[\mathrm{M}+\mathrm{Na}]^{+}=661.2321$, found: 661.2329 .
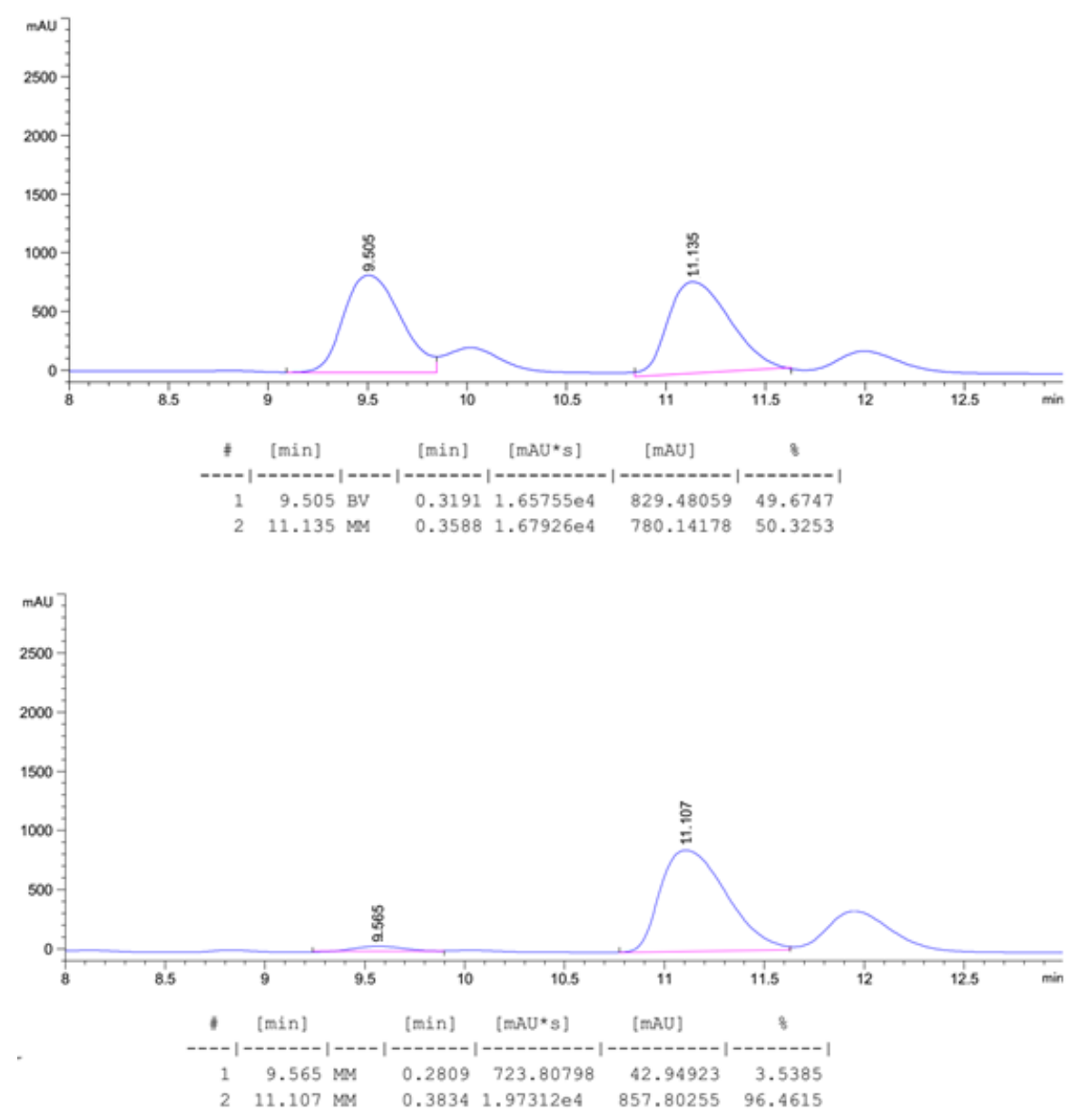

Di-tert-butyl (2'S,3S)-4'-benzoyl-5"-chloro-2,2"-dioxodispiro[indoline-3,1'cyclohexane-2',3"-indolin]-4'-ene-1,1"-dicarboxylate 5h 


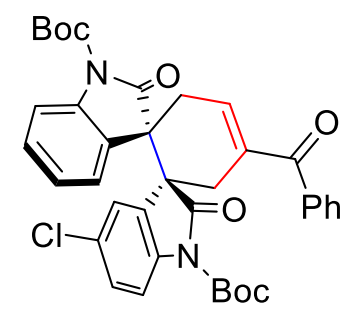

According to general $[4+2]$ annulation procedure, $\mathbf{5} \mathbf{h}$ was obtained in $95 \%$ yield $(62.2$ $\mathrm{mg}$ ) as a deep red solid. The regio-isomers can be separated $\mathrm{rr}=4: 1$, and the major isomer was $91 \%$ ee. $[\alpha]_{\mathrm{D}}^{25}=-42.6\left(\mathrm{c} 1.0, \mathrm{CHCl}_{3}\right)$. The ee was determined by chiral stationary phase HPLC analysis [Daicel Chiralpak IA, isopropanol/hexane $=5 / 95,0.8$ $\mathrm{mL} / \mathrm{min}, \lambda=254 \mathrm{~nm}, \mathrm{t}_{\mathrm{R}}$ (major) $=29 \mathrm{~min}, \mathrm{t}_{\mathrm{R}}($ minor $\left.)=25.4 \mathrm{~min}\right] .{ }^{1} \mathrm{H} \mathrm{NMR}(500 \mathrm{MHz}$, $\left.\mathrm{CDCl}_{3}\right) \delta 7.83(\mathrm{dd}, J=5.3,3.2 \mathrm{~Hz}, 2 \mathrm{H}), 7.59(\mathrm{dd}, J=13.9,13.0 \mathrm{~Hz}, 2 \mathrm{H}), 7.51-7.46$ $(\mathrm{m}, 3 \mathrm{H}), 7.27(\mathrm{~d}, J=2.2 \mathrm{~Hz}, 1 \mathrm{H}), 7.20(\mathrm{td}, J=6.2,3.0 \mathrm{~Hz}, 2 \mathrm{H}), 7.14(\mathrm{dd}, J=8.7,2.1$ $\mathrm{Hz}, 1 \mathrm{H}), 7.02$ (td, $J=7.6,0.8 \mathrm{~Hz}, 1 \mathrm{H}), 6.82-6.78$ (m, 1H), $3.42-3.28$ (m, 2H), 2.98 $(\mathrm{d}, J=18.4 \mathrm{~Hz}, 1 \mathrm{H}), 2.61(\mathrm{dd}, J=19.6,5.2 \mathrm{~Hz}, 1 \mathrm{H}), 1.71(\mathrm{~s}, 9 \mathrm{H}), 1.67(\mathrm{~s}, 9 \mathrm{H}) .{ }^{13} \mathrm{C}$ NMR $\left(126 \mathrm{MHz}, \mathrm{CDCl}_{3}\right) \delta 196.6,180.0,174.6,148.6,148.4,139.4,139.2,138.3,137.5$, 135.0, 131.6, 129.9, 129.5, 129.5, 129.3, 129.1, 128.1, 126.6, 124.2, 123.4, 122.7, 115.7, 114.5, 85.2, 85.0, 50.1, 49.5, 31.9, 29.6, 28.1, 28.0. HRMS (ESI) m/z calcd for $\mathrm{C}_{37} \mathrm{H}_{35} \mathrm{ClN}_{2} \mathrm{O}_{7}[\mathrm{M}+\mathrm{Na}]^{+}=677.2025$, found: 677.2029 .

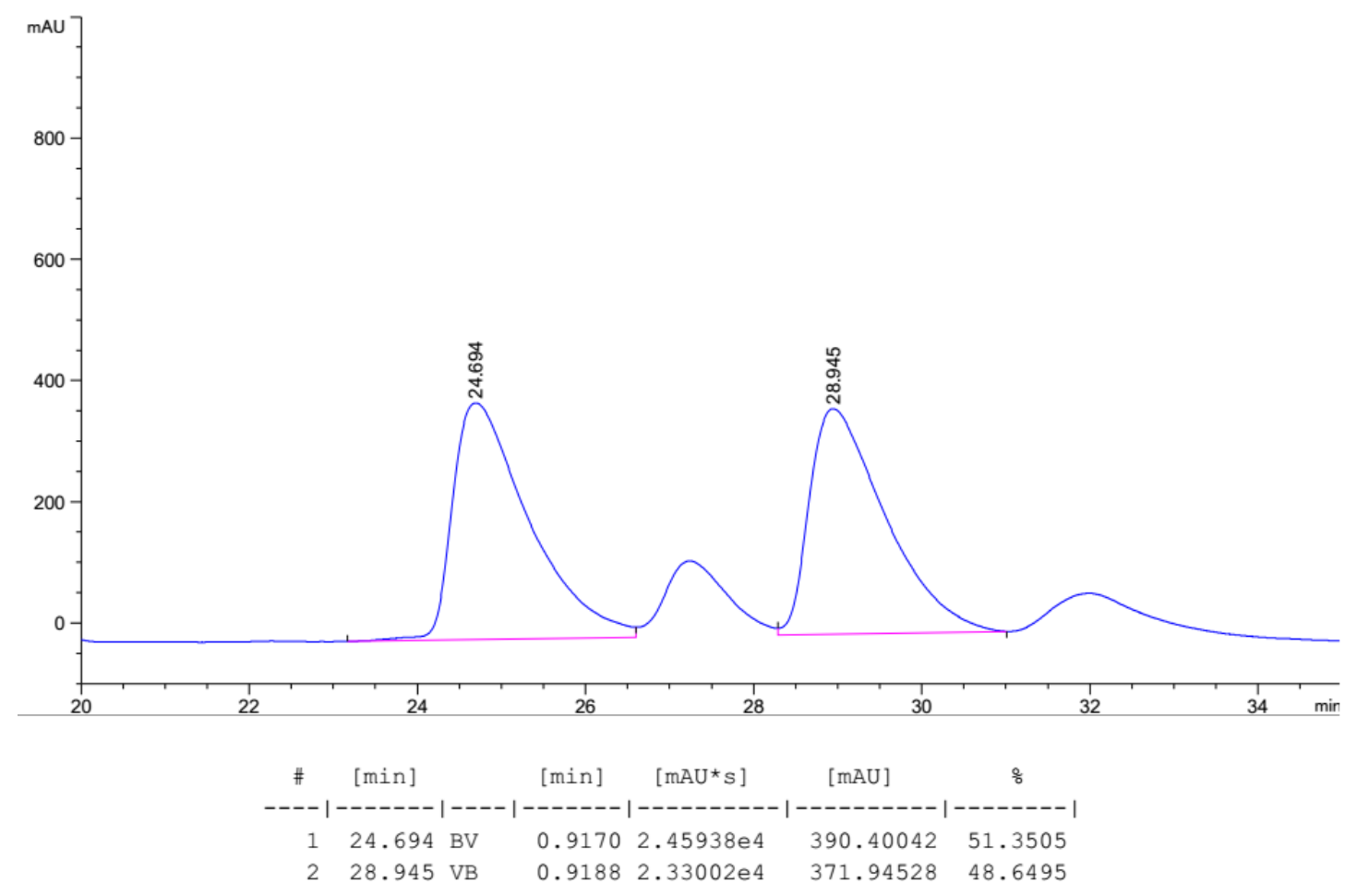




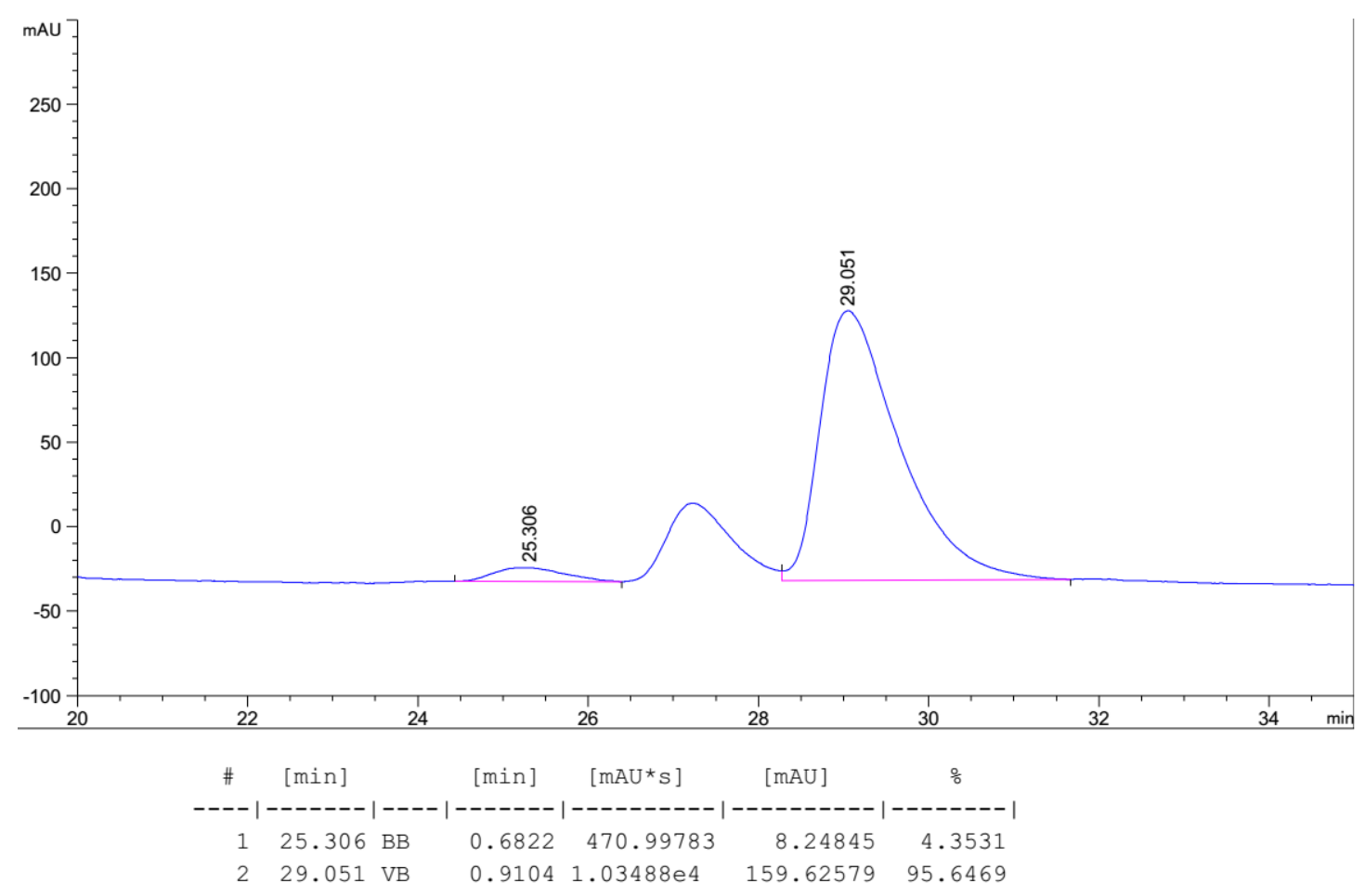

Di-tert-butyl (2'S,3S)-4'-benzoyl-5"-bromo-2,2"-dioxodispiro[indoline-3,1'cyclohexane-2',3"-indolin]-4'-ene-1,1"-dicarboxylate 5i

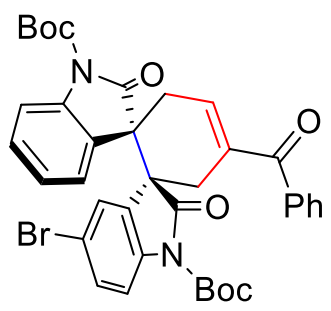

According to general $[4+2]$ annulation procedure, $\mathbf{5 i}$ was obtained in, $92 \%$ yield $(64.3$ $\mathrm{mg}$ ) as a deep red solid and $87 \%$ ee. $\mathrm{rr}=4: 1$ determined by ${ }^{1} \mathrm{H}$ NMR. $[\alpha]_{\mathrm{D}}^{25}=-30.7$ (c $1.0, \mathrm{CHCl}_{3}$ ). The $e e$ was determined by chiral stationary phase HPLC analysis [Daicel Chiralpak ID, isopropanol $/$ hexane $=20 / 80,1.0 \mathrm{~mL} / \mathrm{min}, \lambda=254 \mathrm{~nm}, \mathrm{t}_{\mathrm{R}}($ major $)=13.5$ min, $\mathrm{t}_{\mathrm{R}}($ minor $\left.)=17.6 \mathrm{~min}\right] .{ }^{1} \mathrm{H}$ NMR $\left(500 \mathrm{MHz}, \mathrm{CDCl}_{3}\right) \delta 7.85-7.82(\mathrm{~m}, 2 \mathrm{H}), 7.58$ $(\mathrm{ddd}, J=19.6,11.4,5.5 \mathrm{~Hz}, 3 \mathrm{H}), 7.50-7.38(\mathrm{~m}, 5 \mathrm{H}), 7.22-7.18(\mathrm{~m}, 2 \mathrm{H}), 7.02(\mathrm{t}, J=$ $7.6 \mathrm{~Hz}, 1 \mathrm{H}), 6.82-6.78(\mathrm{~m}, 1 \mathrm{H}), 3.35(\mathrm{dd}, J=23.9,21.9 \mathrm{~Hz}, 2 \mathrm{H}), 3.13(\mathrm{~d}, J=18.4 \mathrm{~Hz}$, $0.22 \mathrm{H}) 2.97(\mathrm{~d}, J=18.4 \mathrm{~Hz}, 0.91 \mathrm{H}), 2.61(\mathrm{dd}, J=19.6,5.4 \mathrm{~Hz}, 1 \mathrm{H}), 1.72(\mathrm{~d}, J=3.9$ $\mathrm{Hz}, 9 \mathrm{H}), 1.67(\mathrm{~d}, J=3.2 \mathrm{~Hz}, 9 \mathrm{H}) .{ }^{13} \mathrm{C} \mathrm{NMR}\left(126 \mathrm{MHz}, \mathrm{CDCl}_{3}\right) \delta 196.6,174.5,174.1$, 148.6, 148.4, 139.4, 139.2, 138.3, 138.0, 135.0, 132.2, 131.6, 129.7, 129.5, 129.5, 129.4, $128.9,128.1,126.6,126.3,124.2,122.7,117.3,116.1,114.5,85.2,85.0,50.1,49.5$, 31.9, 29.7, 28.1, 28.1, 28.1, 28.0. HRMS (ESI) $\mathrm{m} / \mathrm{z}$ calcd for $\mathrm{C}_{37} \mathrm{H}_{35} \mathrm{BrN}_{2} \mathrm{O}_{7}[\mathrm{M}+\mathrm{Na}]^{+}$ $=721.1520$, found: 721.1527 . 
<Chromatogram>

$\mathrm{mV}$

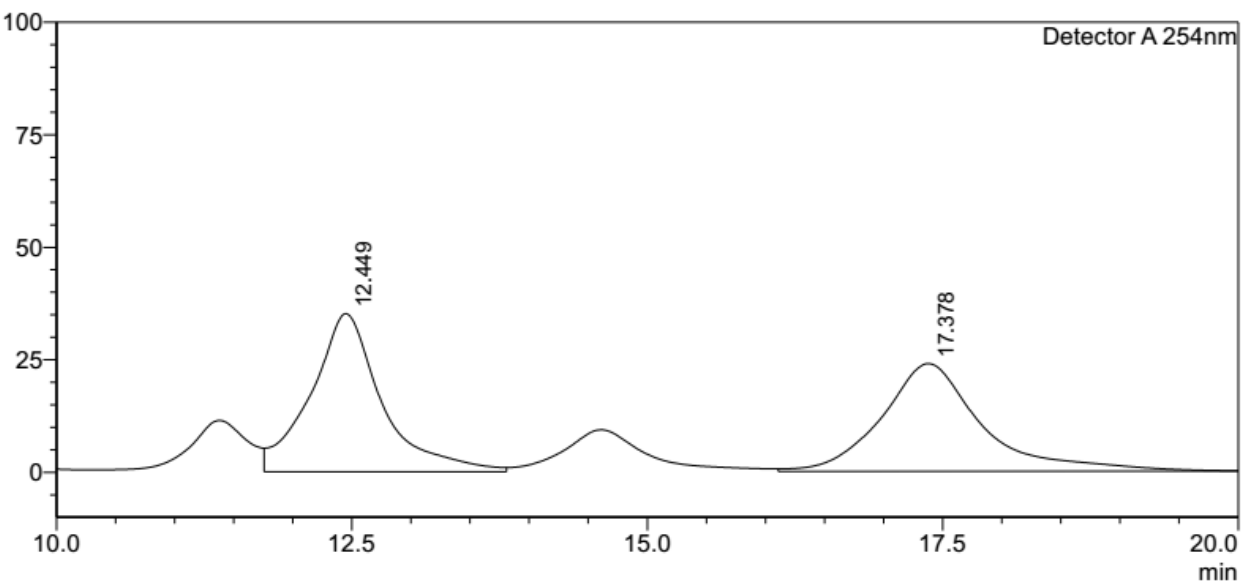

<Peak Table>

Detector A 254nm

\begin{tabular}{|r|r|r|r|r|r|r|r|}
\hline Peak\# & Ret. Time & \multicolumn{1}{|c|}{ Area } & \multicolumn{1}{c|}{ Height } & \multicolumn{1}{c|}{ Conc. } & Unit & Mark & Name \\
\hline 1 & 12.449 & 1471279 & 35151 & 50.823 & & & \\
\hline 2 & 17.378 & 1423625 & 23974 & 49.177 & & & \\
\hline Total & & 2894904 & 59125 & & & &
\end{tabular}

<Chromatogram>

$\mathrm{mV}$

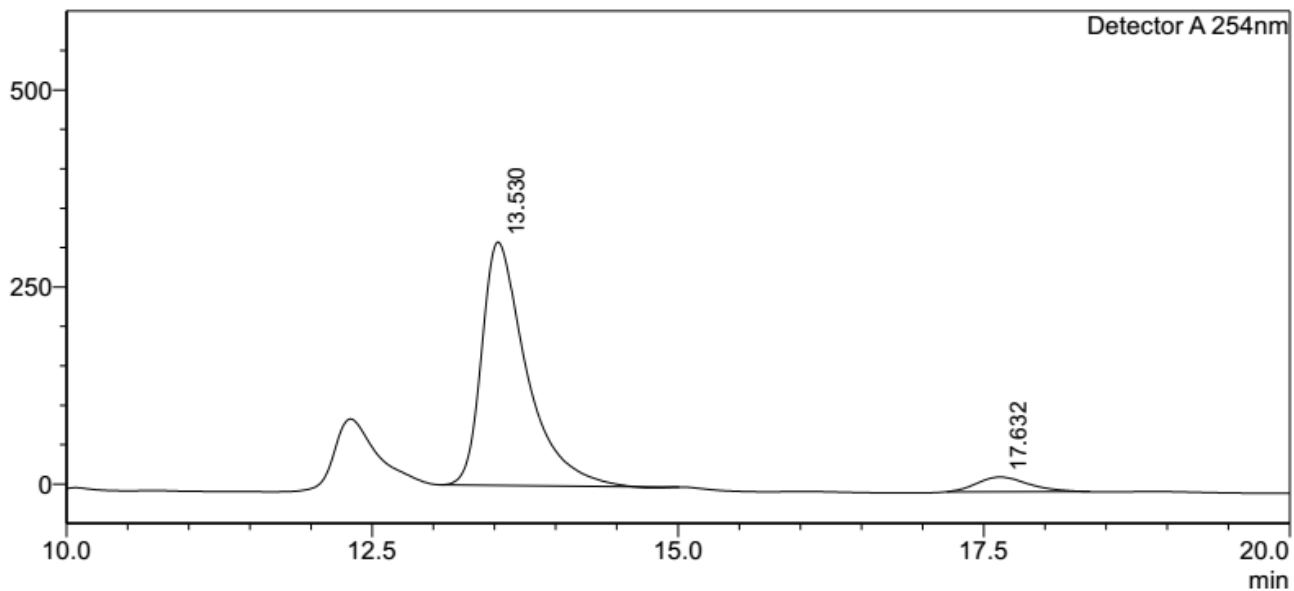

<Peak Table>

\begin{tabular}{|c|c|c|c|c|c|c|c|}
\hline & Area & Height & Conc. & Unit & Mark & Name \\
\hline \multicolumn{2}{|c|}{\begin{tabular}{r|r} 
Peak\# & Ret. Time \\
1 & 13.530
\end{tabular}} & 7859923 & 308599 & 93.477 & & $\mathrm{M}$ & \\
\hline 2 & 17.632 & 548454 & 18636 & 6.523 & & $M$ & \\
\hline Total| & & 8408376 & 327235 & & & & \\
\hline
\end{tabular}

Di-tert-butyl (2'S,3S)-4'-benzoyl-5-methoxy-2,2"-dioxodispiro[indoline-3,1'cyclohexane-2',3"-indolin]-4'-ene-1,1"-dicarboxylate $\mathbf{5 j}$<smiles>COc1ccc2c(c1)[C@@]1(CC=C(C(=O)c3ccccc3)CC1(C(=O)OC(C)(C)C)C(=O)N(C(=O)OC(C)(C)C)c1ccccc1)c1ccccc1-2</smiles>

According to general [4 +2$]$ annulation procedure, longer reaction time 12 hours is 
needed. $\mathbf{5} \mathbf{j}$ was obtained in $94 \%$ yield $(61.1 \mathrm{mg})$ as a deep red solid and $95 \%$ ee. $\mathrm{rr}=$ 1.8:1 determined by ${ }^{1} \mathrm{H}$ NMR. $[\alpha]_{\mathrm{D}}^{25}=-84.7\left(\mathrm{c} 1.0, \mathrm{CHCl}_{3}\right)$. The ee was determined by chiral stationary phase HPLC analysis [Daicel Chiralpak ID, isopropanol/hexane = $10 / 90,1.0 \mathrm{~mL} / \mathrm{min}, \lambda=254 \mathrm{~nm}, \mathrm{t}_{\mathrm{R}}$ (major) $=23.3 \mathrm{~min}, \mathrm{t}_{\mathrm{R}}($ minor $\left.)=35.7 \mathrm{~min}\right] .{ }^{1} \mathrm{H} \mathrm{NMR}$ $\left(500 \mathrm{MHz}, \mathrm{CDCl}_{3}\right) \delta 7.83(\mathrm{dd}, J=8.1,0.8 \mathrm{~Hz}, 2 \mathrm{H}), 7.58-7.52(\mathrm{~m}, 2 \mathrm{H}), 7.49-7.42$ $(\mathrm{m}, 3 \mathrm{H}), 7.24(\mathrm{dd}, J=8.1,7.4 \mathrm{~Hz}, 1 \mathrm{H}), 7.18(\mathrm{td}, J=8.2,1.2 \mathrm{~Hz}, 1 \mathrm{H}), 7.04-7.00(\mathrm{~m}$, $1 \mathrm{H}), 6.84(\mathrm{dd}, J=10.5,2.6 \mathrm{~Hz}, 1 \mathrm{H}), 6.81-6.78(\mathrm{~m}, 1 \mathrm{H}), 6.68(\mathrm{dd}, J=9.0,2.7 \mathrm{~Hz}, 1 \mathrm{H})$, $3.75(\mathrm{~s}, 1.9 \mathrm{H}), 3.74(\mathrm{~s}, 1.1 \mathrm{H}), 3.43-3.32(\mathrm{~m}, 2 \mathrm{H}), 2.96(\mathrm{dd}, J=18.5,3.9 \mathrm{~Hz}, 1 \mathrm{H}), 2.59$ $(\mathrm{dd}, J=19.6,5.2 \mathrm{~Hz}, 1 \mathrm{H}), 1.67(\mathrm{~d}, J=2.0 \mathrm{~Hz}, 18 \mathrm{H}) .{ }^{13} \mathrm{C} \mathrm{NMR}\left(126 \mathrm{MHz}, \mathrm{CDCl}_{3}\right) \delta$ 196.8, 175.0, 156.7, 148.7, 139.5, 139.1, 138.4, 135.4, 132.1, 131.6, 129.5, 129.3, 128.5, $128.1,127.1,124.2,122.9,122.8,115.5,114.4,114.3,109.0,84.9,84.4,55.4,50.3$, 50.1, 49.9, 49.6, 32.2, 32.1, 29.9, 28.1, 28.0. HRMS (ESI) m/z calcd for $\mathrm{C}_{38} \mathrm{H}_{38} \mathrm{~N}_{2} \mathrm{O}_{8}$ $[\mathrm{M}+\mathrm{Na}]^{+}=673.2520$, found: 673.2525 .

$\mathrm{mV}$

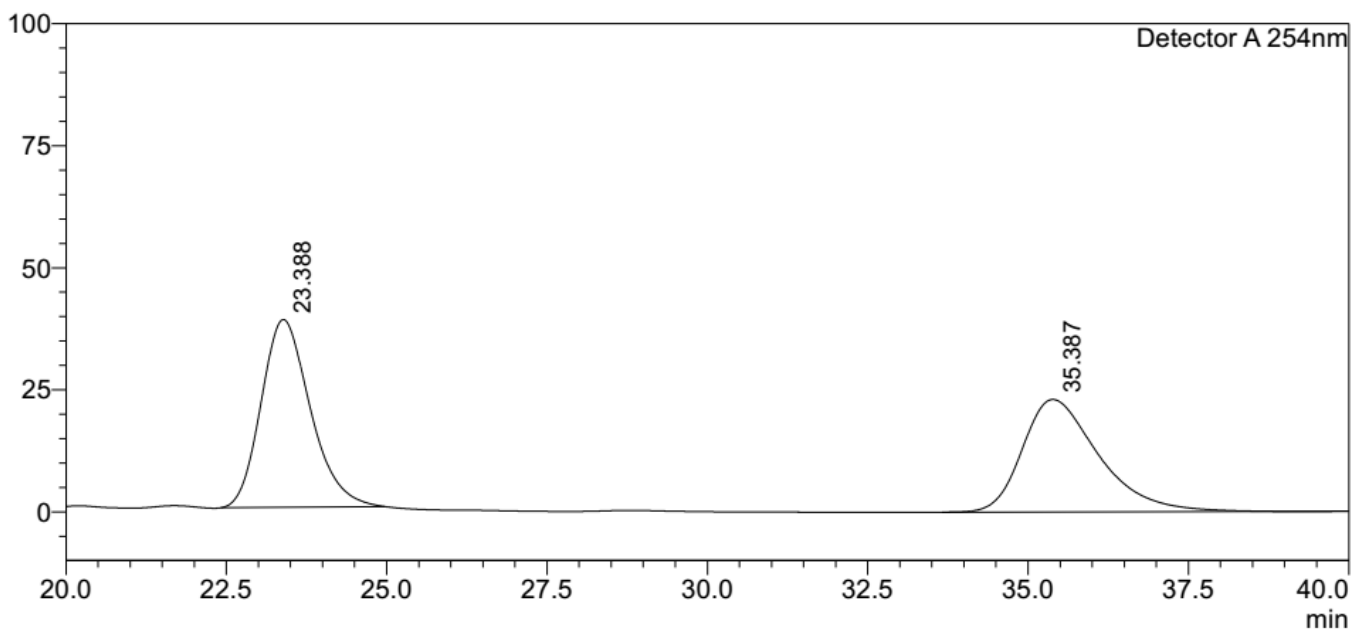

<Peak Table>

Detector A 254nm

\begin{tabular}{|r|r|r|r|r|r|r|r|}
\hline Peak\# & Ret. Time & Area & Height & Conc. & Unit & Mark & Name \\
\hline 1 & 23.388 & 2056550 & 38484 & 51.925 & & $\mathrm{M}$ & \\
\hline 2 & 35.387 & 1904031 & 23041 & 48.075 & & $\mathrm{M}$ & \\
\hline Total & & 3960581 & 61525 & & & & \\
\hline
\end{tabular}




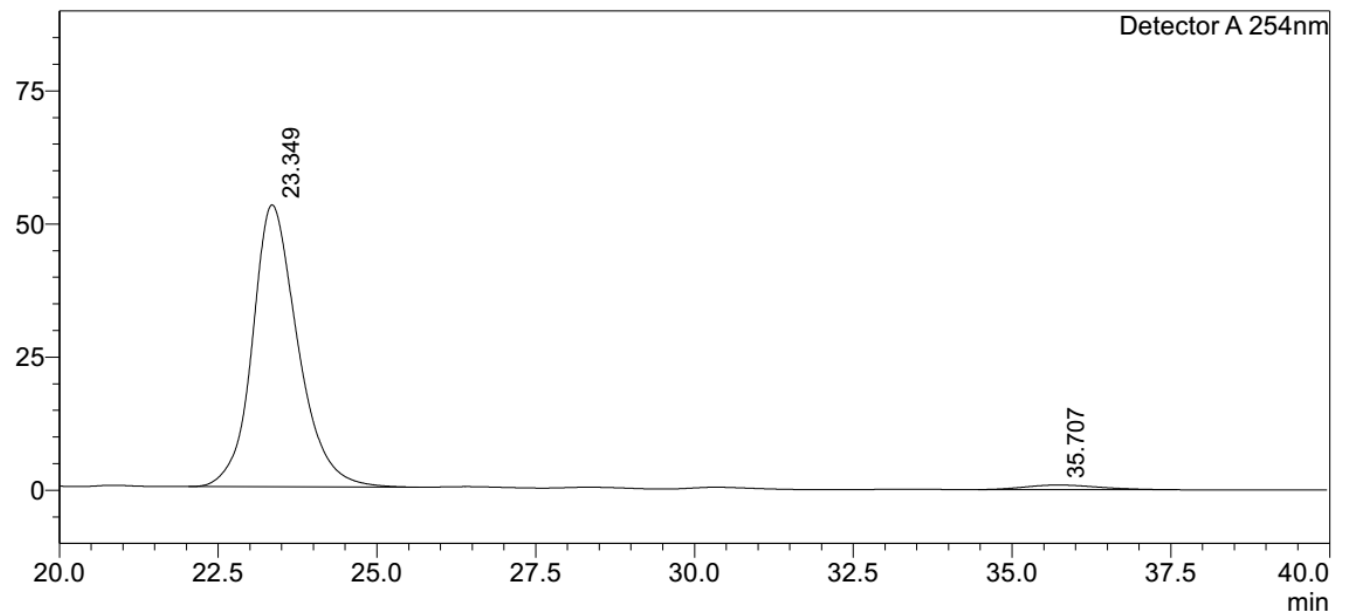

$<$ Peak Table>

Detector A 254nm Peak\# Ret. Time

\begin{tabular}{|r|r|r|r|r|r|r|r|}
\hline Peak\# & Ret. Time & \multicolumn{1}{c|}{ Area } & \multicolumn{1}{c|}{ Height } & Conc. & Unit & Mark & Name \\
\hline 1 & 23.349 & 2639481 & 52953 & 97.383 & & & \\
\hline 2 & 35.707 & 70922 & 871 & 2.617 & & & \\
\hline Total & & 2710404 & 53824 & & & & \\
\hline
\end{tabular}

Di-tert-butyl (2'S,3S)-4'-benzoyl-5,7-dimethyl-2,2"-dioxodispiro[indoline-3,1'cyclohexane-2',3"-indolin]-4'-ene-1,1"-dicarboxylate 5k<smiles>Cc1cc(C)c(C2(C(=O)c3ccccc3)CC=C(C(=O)c3ccccc3)CC2)c(C)c1C(=O)OC(C)(C)C</smiles>

According to general [4 +2$]$ annulation procedure, longer reaction time 12 hours is needed. $\mathbf{5 k}$ was obtained in $84 \%$ yield $(54.4 \mathrm{mg})$ as a deep red solid and $96 \%$ ee. $\mathrm{rr}=$ 5.4:1 determined by ${ }^{1} \mathrm{H}$ NMR. $[\alpha]_{\mathrm{D}}^{25}=-227.7\left(\mathrm{c} 1.0, \mathrm{CHCl}_{3}\right)$. The ee was determined by chiral stationary phase HPLC analysis [Daicel Chiralpak IA, isopropanol/hexane $=5 / 95$, $0.8 \mathrm{~mL} / \mathrm{min}, \lambda=254 \mathrm{~nm}, \mathrm{t}_{\mathrm{R}}$ (major) $=10.8 \mathrm{~min}, \mathrm{t}_{\mathrm{R}}$ (minor) $\left.=9.0 \mathrm{~min}\right] .{ }^{1} \mathrm{H} \mathrm{NMR}(500$ $\left.\mathrm{MHz}, \mathrm{CDCl}_{3}\right) \delta 7.87-7.79(\mathrm{~m}, 2 \mathrm{H}), 7.54(\mathrm{~d}, J=7.6 \mathrm{~Hz}, 2 \mathrm{H}), 7.47(\mathrm{t}, J=7.5 \mathrm{~Hz}, 2 \mathrm{H})$, $7.24(\mathrm{~d}, J=7.6 \mathrm{~Hz}, 1 \mathrm{H}), 7.21-7.16(\mathrm{~m}, 1 \mathrm{H}), 7.06-7.01(\mathrm{~m}, 1 \mathrm{H}), 6.90(\mathrm{~s}, 1 \mathrm{H}), 6.80-$ $6.78(\mathrm{~m}, 1 \mathrm{H}), 6.76(\mathrm{~s}, 1 \mathrm{H}), 3.39(\mathrm{t}, J=18.5 \mathrm{~Hz}, 2 \mathrm{H}), 2.92(\mathrm{~d}, J=18.0 \mathrm{~Hz}, 1 \mathrm{H}), 2.59$ $(\mathrm{dd}, J=18.8,5.1 \mathrm{~Hz}, 1 \mathrm{H}), 2.23(\mathrm{~s}, 0.47 \mathrm{H}), 2.22(\mathrm{~s}, 2.53 \mathrm{H}), 2.01(\mathrm{~s}, 0.47 \mathrm{H}), 1.99(\mathrm{~s}$, $2.52 \mathrm{H}), 1.68(\mathrm{~d}, J=1.1 \mathrm{~Hz}, 18 \mathrm{H}) .{ }^{13} \mathrm{C} \mathrm{NMR}\left(101 \mathrm{MHz}, \mathrm{CDCl}_{3}\right) \delta 196.8,175.6,175.1$, $148.8,148.7,139.6,138.9,138.5,135.4,135.4,133.6,132.6,131.5,129.5,129.0,128.3$, $128.0,127.4,124.2,123.4,122.4,121.2,114.2,84.8,84.5,49.9,49.8,32.3,30.0,28.1$, 28.0, 27.8, 27.7, 20.8, 19.0. HRMS (ESI) $\mathrm{m} / \mathrm{z}$ calcd for $\mathrm{C}_{39} \mathrm{H}_{40} \mathrm{~N}_{2} \mathrm{O}_{7}[\mathrm{M}+\mathrm{Na}]^{+}=$ 671.2728, found: 671.2733 . 

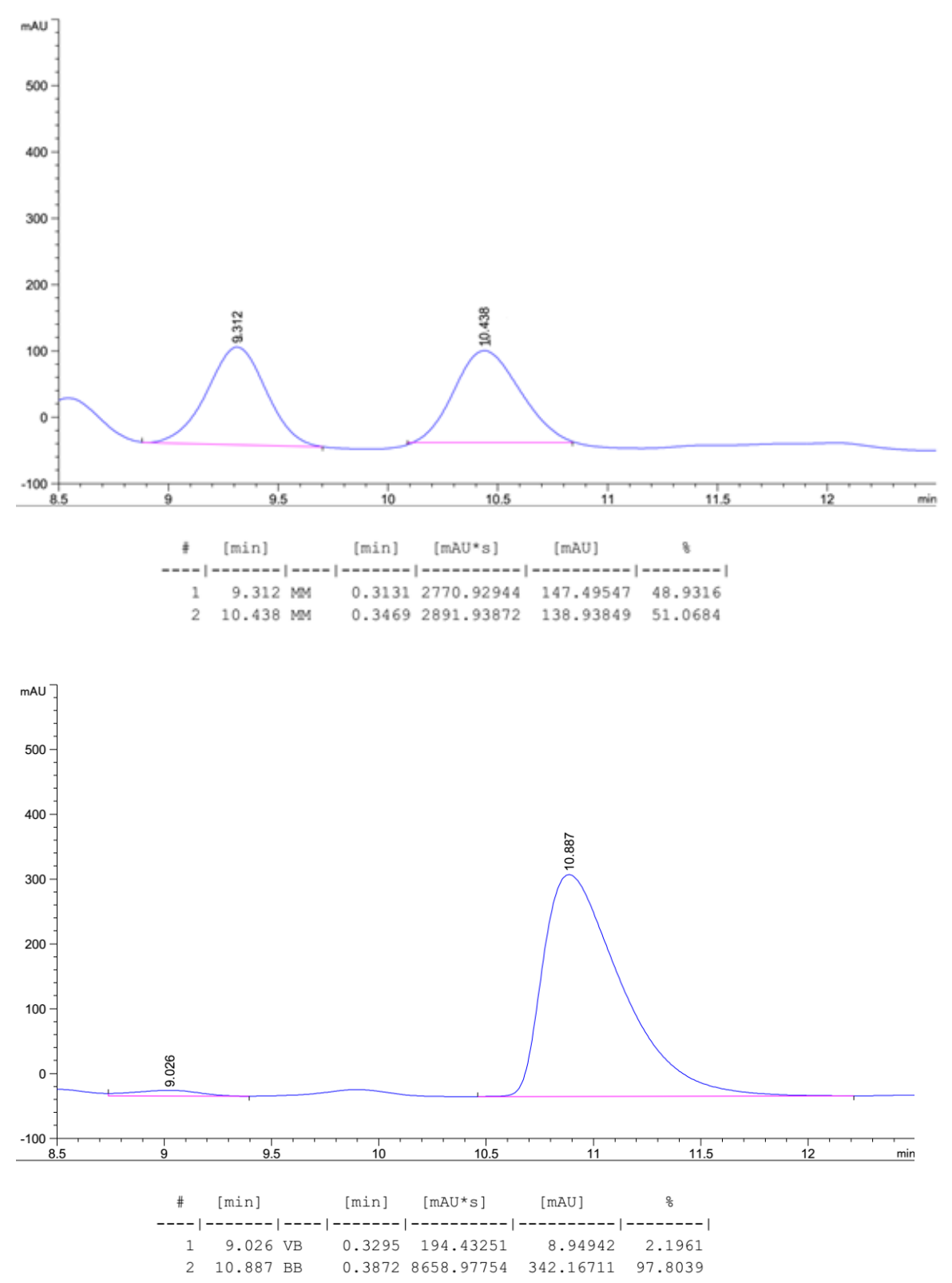

Di-tert-butyl (2'S,3S)-4'-benzoyl-6"-fluoro-2,2"-dioxodispiro[indoline-3,1'cyclohexane-2',3"-indolin]-4'-ene-1,1"-dicarboxylate 51

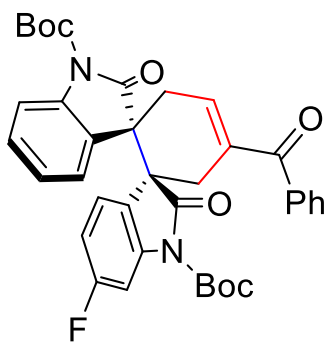

According to general [4+2] annulation procedure, $5 \mathbf{l}$ was obtained in $88 \%$ yield (56.2 $\mathrm{mg}$ ) as a deep red solid and $98 \%$ ee. $\mathrm{rr}>20: 1$ determined by ${ }^{1} \mathrm{H}$ NMR, $[\alpha]_{\mathrm{D}}^{25}=-97.4(\mathrm{c}$ $1.0, \mathrm{CHCl}_{3}$ ). After deprotected the two boc groups, the ee was determined by chiral stationary phase HPLC analysis [Daicel Chiralpak IC, isopropanol/hexane $=40 / 60,1.0$ $\mathrm{mL} / \mathrm{min}, \lambda=254 \mathrm{~nm}, \mathrm{t}_{\mathrm{R}}($ major $)=41.8 \mathrm{~min}, \mathrm{t}_{\mathrm{R}}($ minor $\left.)=31 \mathrm{~min}\right] .{ }^{1} \mathrm{H} \mathrm{NMR}(500 \mathrm{MHz}$, 
$\left.\mathrm{CDCl}_{3}\right) \delta 7.82(\mathrm{~d}, J=7.2 \mathrm{~Hz}, 2 \mathrm{H}), 7.50(\mathrm{dt}, J=39.7,7.1 \mathrm{~Hz}, 5 \mathrm{H}), 7.32(\mathrm{dd}, J=7.5,5.3$ $\mathrm{Hz}, 1 \mathrm{H}), 7.27-7.16(\mathrm{~m}, 3 \mathrm{H}), 7.07-6.98(\mathrm{~m}, 1 \mathrm{H}), 6.79(\mathrm{~s}, 1 \mathrm{H}), 6.71(\mathrm{~s}, 1 \mathrm{H}), 3.35(\mathrm{t}, J$ $=16.9 \mathrm{~Hz}, 2 \mathrm{H}), 2.96(\mathrm{~d}, J=18.3 \mathrm{~Hz}, 1 \mathrm{H}), 2.68-2.54(\mathrm{~m}, 1 \mathrm{H}), 1.67(\mathrm{~s}, 18 \mathrm{H}) .{ }^{13} \mathrm{C} \mathrm{NMR}$ $\left(126 \mathrm{MHz}, \mathrm{CDCl}_{3}\right) \delta 196.7,174.9,174.7,148.3,139.3,139.2,138.3,135.2,131.6$, $129.5,129.4,128.1,127.0,126.8,124.2,122.8,122.7,114.6,110.9,110.7,103.5,103.3$, 85.2, 85.1, 84.9, 84.8, 50.1, 49.9, 49.7, 49.4, 31.9, 29.6, 28.1, 28.1. HRMS (ESI) m/z calcd for $\mathrm{C}_{37} \mathrm{H}_{35} \mathrm{FN}_{2} \mathrm{O}_{7}[\mathrm{M}+\mathrm{Na}]^{+}=661.2321$, found: 661.2328 .

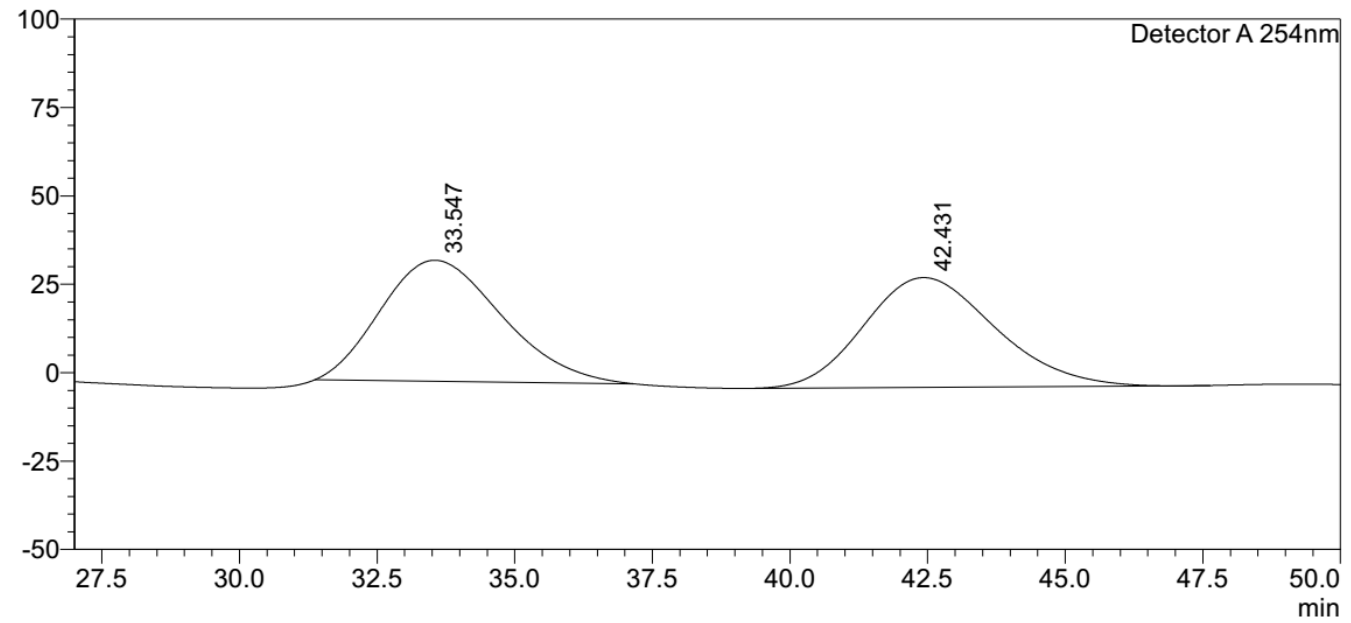

<Peak Table>

Detector A 254nm

\begin{tabular}{|r|r|r|r|r|r|r|r|}
\hline Peak\# & Ret. Time & \multicolumn{1}{|c|}{ Area } & Height & Conc. & Unit & Mark & Name \\
\hline 1 & 33.547 & 5232376 & 34230 & 50.457 & & $\mathrm{M}$ & \\
\hline 2 & 42.431 & 5137513 & 31018 & 49.543 & & $\mathrm{M}$ & \\
\hline Total & & 10369889 & 65248 & & & & \\
\hline
\end{tabular}

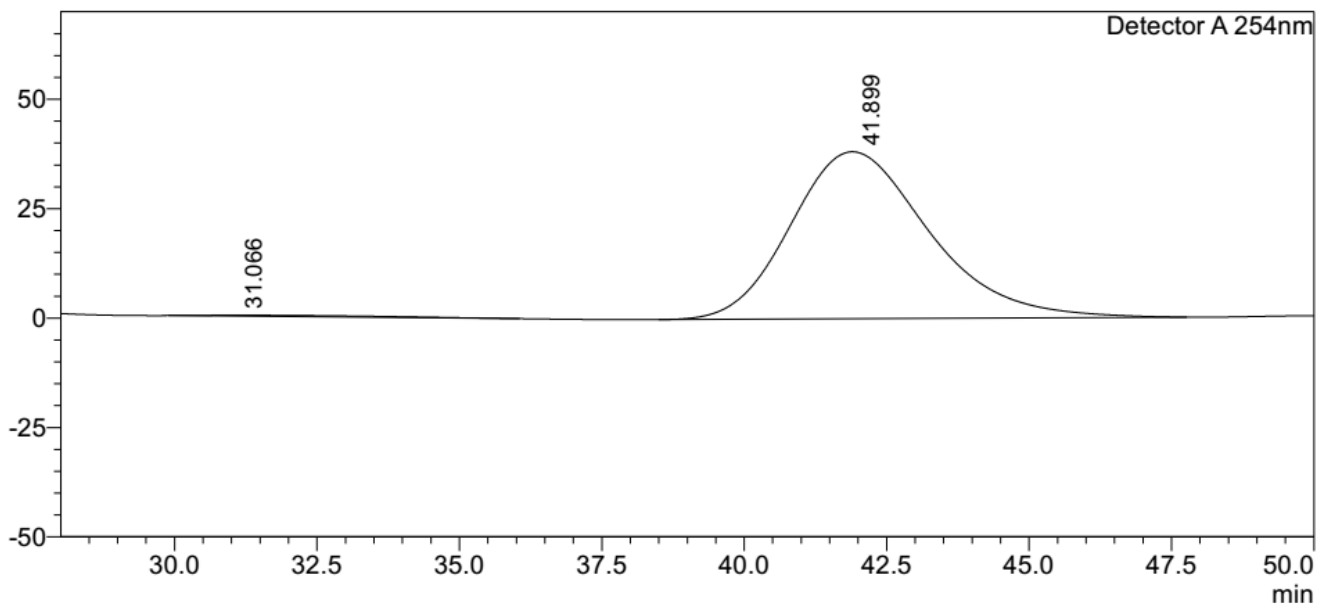

<Peak Table>

Detector A 254nm

\begin{tabular}{|r|r|r|r|r|r|r|r|}
\hline Peak\# & Ret. Time & \multicolumn{1}{c|}{ Area } & \multicolumn{1}{c|}{ Height } & Conc. & Unit & Mark & Name \\
\hline 1 & 31.066 & 67703 & 231 & 1.036 & & $\mathrm{M}$ & \\
\hline 2 & 41.899 & 6466858 & 38167 & 98.964 & & & \\
\hline Total & & 6534561 & 38398 & & & & \\
\hline
\end{tabular}


Di-tert-butyl (2'S,3S)-4'-benzoyl-7"-chloro-2,2"-dioxodispiro[indoline-3,1'cyclohexane-2',3"-indolin]-4'-ene-1,1"-dicarboxylate $\mathbf{5 m}$<smiles>CC(C)(C)OC(=O)N1CC2(C(=O)C(C(=O)c3ccccc3)=CCC23C(=O)N(C(=O)OC(C)(C)C)c2ccccc23)c2ccccc21</smiles>

According to general $[4+2]$ annulation procedure, $\mathbf{5 m}$ was obtained in $81 \%$ yield $(53$ $\mathrm{mg}$ ) as a deep red solid and 96\% ee. $\mathrm{rr}>3.5: 1$ determined by ${ }^{1} \mathrm{H}$ NMR. $[\alpha]_{\mathrm{D}}^{25}=-99.7$ (c $1.0, \mathrm{CHCl}_{3}$ ). The $e e$ was determined by chiral stationary phase HPLC analysis [Daicel Chiralpak ID, isopropanol $/$ hexane $=20 / 80,0.8 \mathrm{~mL} / \mathrm{min}, \lambda=254 \mathrm{~nm}, \mathrm{t}_{\mathrm{R}}$ (major) $=28$ min, $\mathrm{t}_{\mathrm{R}}($ minor $\left.)=34 \mathrm{~min}\right] .{ }^{1} \mathrm{H} \mathrm{NMR}\left(500 \mathrm{MHz}, \mathrm{CDCl}_{3}\right) \delta 7.82(\mathrm{~d}, J=7.1 \mathrm{~Hz}, 2 \mathrm{H}), 7.59$ $-7.52(\mathrm{~m}, 2 \mathrm{H}), 7.47(\mathrm{t}, J=7.3 \mathrm{~Hz}, 2 \mathrm{H}), 7.28(\mathrm{~d}, J=8.0 \mathrm{~Hz}, 1 \mathrm{H}), 7.21(\mathrm{t}, J=7.7 \mathrm{~Hz}$, $1 \mathrm{H}), 7.12(\mathrm{dd}, J=20.5,7.4 \mathrm{~Hz}, 3 \mathrm{H}), 6.94(\mathrm{~d}, J=7.8 \mathrm{~Hz}, 1 \mathrm{H}), 6.80(\mathrm{~s}, 0.24 \mathrm{H}), 6.77(\mathrm{~s}$, $0.76 \mathrm{H}), 3.49-3.31(\mathrm{~m}, 2 \mathrm{H}), 2.96(\mathrm{~d}, J=18.2 \mathrm{~Hz}, 1 \mathrm{H}), 2.70-2.57(\mathrm{~m}, 1 \mathrm{H}), 1.68(\mathrm{~s}$, $18 \mathrm{H}) .{ }^{13} \mathrm{C} \mathrm{NMR}\left(126 \mathrm{MHz}, \mathrm{CDCl}_{3}\right) \delta 196.6,174.9,174.7,148.6,147.5,138.7,138.3$, 136.5, 135.5, 131.6, 130.9, 130.5, 129.5, 129.3, 128.1, 126.9, 124.7, 124.6, 123.5, 121.4, 117.6, 114.4, 85.9, 84.7, 50.3, 49.7, 31.9, 30.0, 29.7, 28.1, 27.6. HRMS (ESI) m/z calcd for $\mathrm{C}_{37} \mathrm{H}_{35} \mathrm{ClN}_{2} \mathrm{O}_{7}[\mathrm{M}+\mathrm{Na}]^{+}=677.2025$, found: 677.2031 .

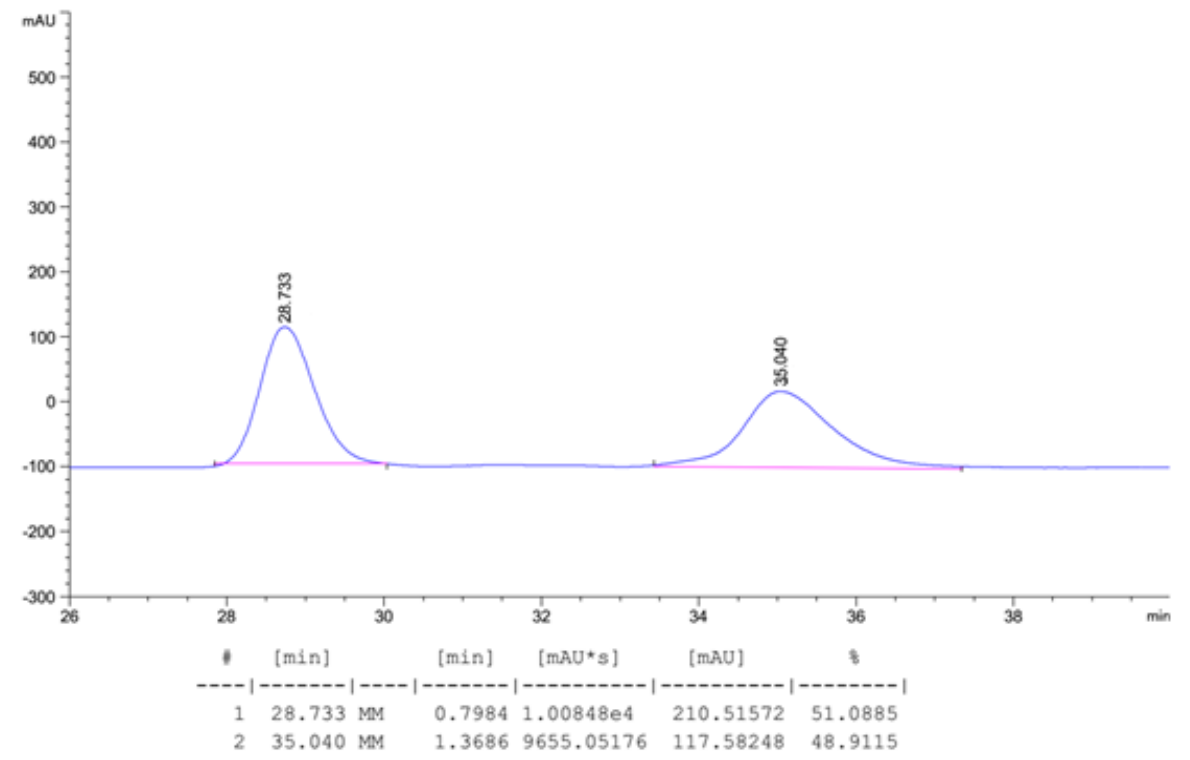




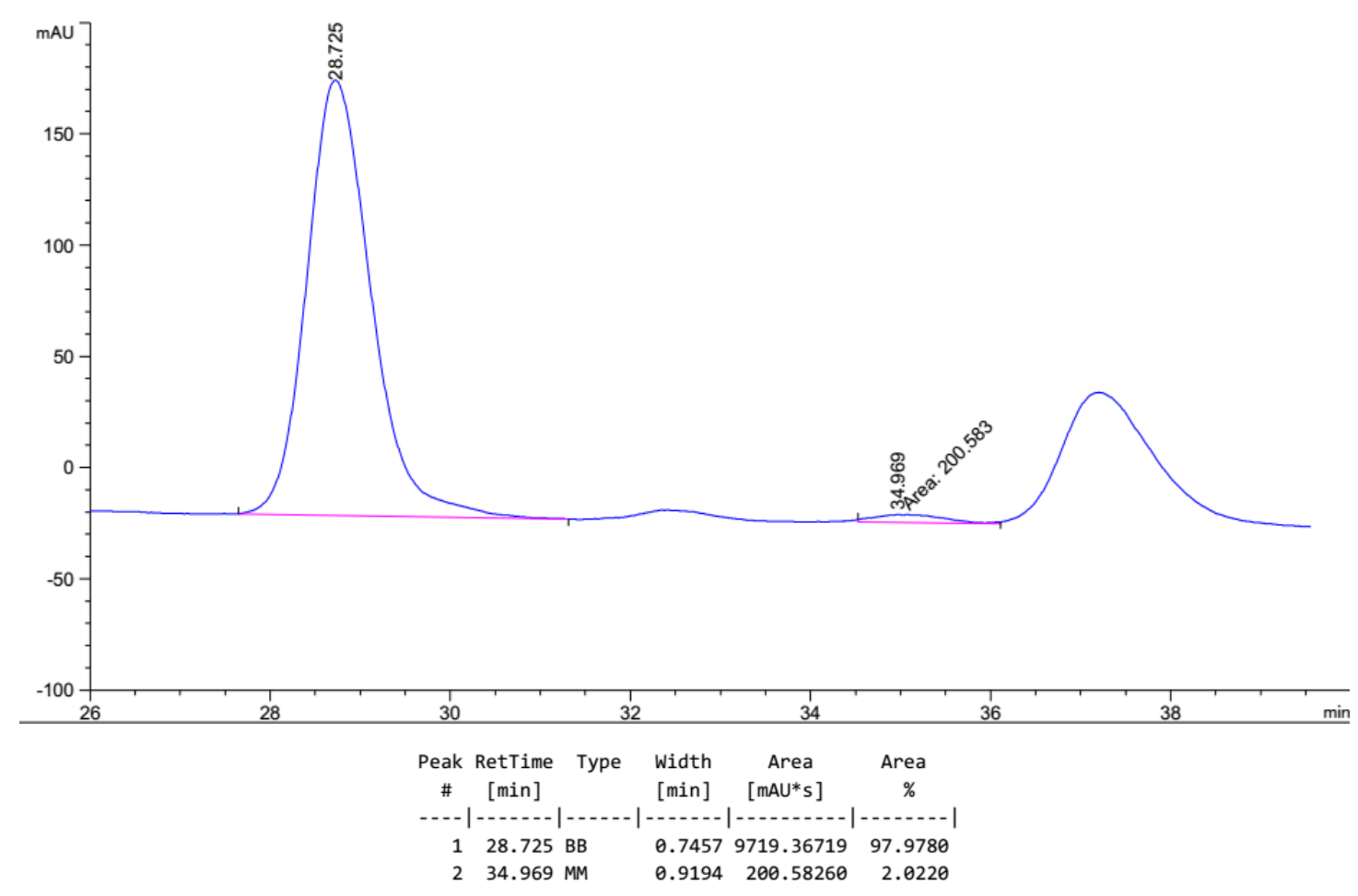

Di-tert-butyl (2'S,3S)-4'-(2-methylbenzoyl)-2,2"-dioxodispiro[indoline-3,1'cyclohexane-2',3"-indolin]-4'-ene-1,1"-dicarboxylate 5n

Boc

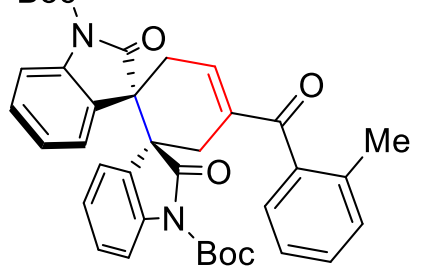

According to general $[4+2]$ annulation procedure, $\mathbf{5 n}$ was obtained in 95\% yield $(60.2$ $\mathrm{mg}$ ) as a deep red solid and $90 \%$ ee. $[\alpha]_{\mathrm{D}}^{25}=-210.6\left(\mathrm{c} 1.0, \mathrm{CHCl}_{3}\right)$. The ee was determined by chiral stationary phase HPLC analysis [Daicel Chiralpak IA, isopropanol $/$ hexane $=5 / 95,0.8 \mathrm{~mL} / \mathrm{min}, \lambda=254 \mathrm{~nm}, \mathrm{t}_{\mathrm{R}}$ (major) $=8.5 \mathrm{~min}, \mathrm{t}_{\mathrm{R}}$ (minor) $=7.7 \mathrm{~min}] .{ }^{1} \mathrm{H}$ NMR $\left(500 \mathrm{MHz}, \mathrm{CDCl}_{3}\right) \delta 7.56-7.47(\mathrm{~m}, 2 \mathrm{H}), 7.47-7.41(\mathrm{~m}, 1 \mathrm{H})$, $7.38-7.32(\mathrm{~m}, 1 \mathrm{H}), 7.29-7.15(\mathrm{~m}, 6 \mathrm{H}), 7.05-6.97(\mathrm{~m}, 2 \mathrm{H}), 6.71(\mathrm{~s}, 1 \mathrm{H}), 3.32(\mathrm{t}, J$ $=18.1 \mathrm{~Hz}, 2 \mathrm{H}), 3.08-2.95(\mathrm{~m}, 1 \mathrm{H}), 2.54(\mathrm{~d}, J=20.3 \mathrm{~Hz}, 1 \mathrm{H}), 2.43(\mathrm{~d}, J=3.9 \mathrm{~Hz}, 3 \mathrm{H})$, $1.72-1.67(\mathrm{~m}, 18 \mathrm{H}) .{ }^{13} \mathrm{C}$ NMR $\left(126 \mathrm{MHz}, \mathrm{CDCl}_{3}\right) \delta 198.9,175.0,174.9,148.5,141.7$, 139.2, 139.0, 139.0, 136.7, 136.3, 130.6, 129.5, 129.3, 129.2, 128.1, 127.2, 126.9, 125.0, 124.1, 122.9, 122.8, 114.5, 84.7, 84.6, 50.2, 49.8, 32.0, 29.7, 28.8, 28.1, 19.7. HRMS (ESI) $\mathrm{m} / \mathrm{z}$ calcd for $\mathrm{C}_{38} \mathrm{H}_{38} \mathrm{~N}_{2} \mathrm{O}_{7}[\mathrm{M}+\mathrm{Na}]^{+}=657.2571$, found: 657.2575 . 

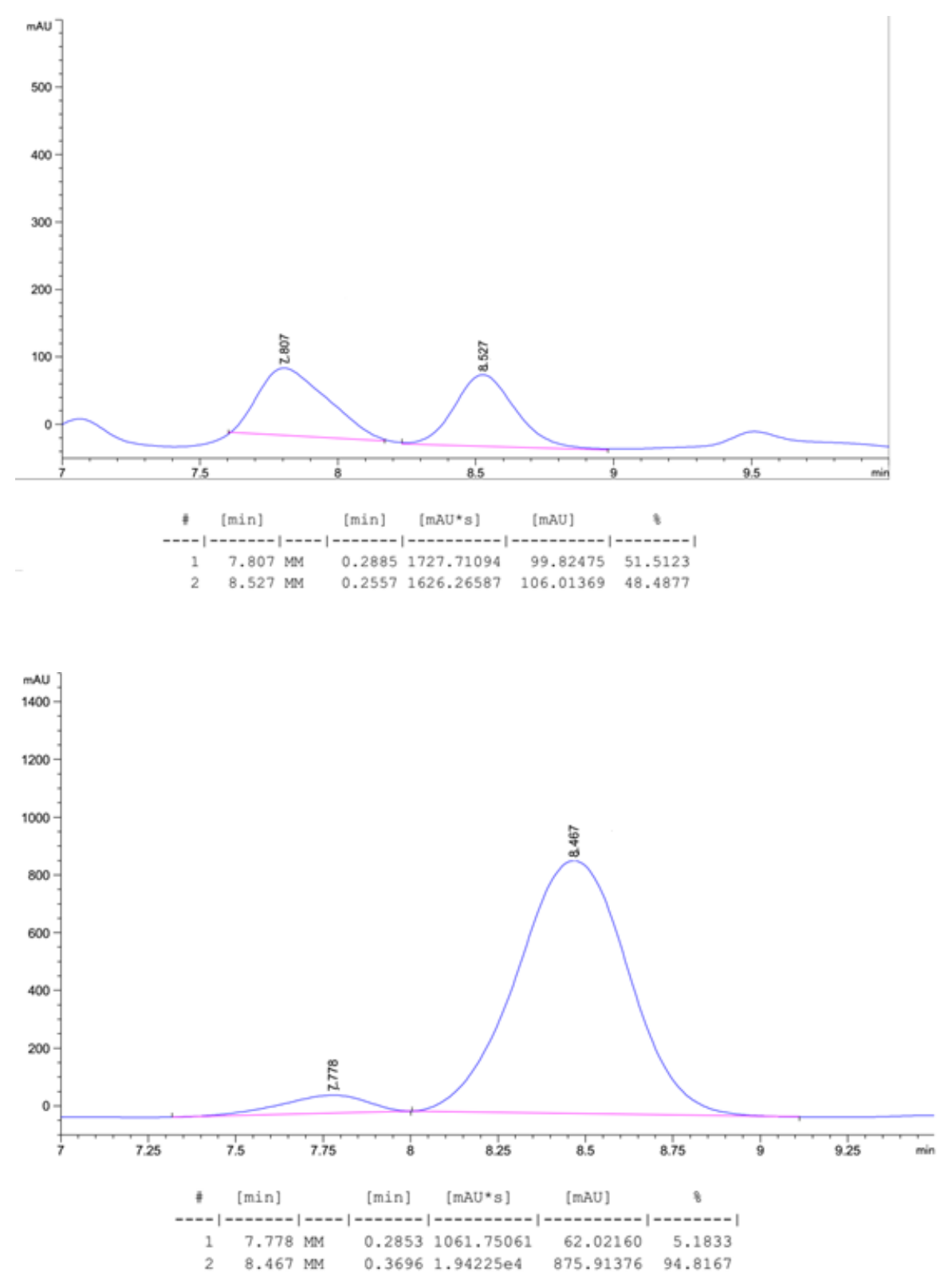

Di-tert-butyl (2'S,3S)-4'-(3-methylbenzoyl)-2,2"-dioxodispiro[indoline-3,1'cyclohexane-2',3"-indolin]-4'-ene-1,1"-dicarboxylate 50

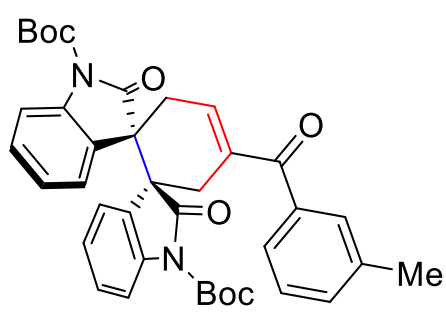

According to general [4+2] annulation procedure, 50 was obtained in $95 \%$ yield $(60.3$ $\mathrm{mg})$ as a deep red solid and $93 \%$ ee. $[\alpha]_{\mathrm{D}}^{25}=-213.7\left(\mathrm{c} 1.0, \mathrm{CHCl}_{3}\right)$. The ee was determined by chiral stationary phase HPLC analysis [Daicel Chiralpak IA, isopropanol $/$ hexane $=5 / 95,0.8 \mathrm{~mL} / \mathrm{min}, \lambda=254 \mathrm{~nm}, \mathrm{t}_{\mathrm{R}}($ major $)=10.5 \mathrm{~min}, \mathrm{t}_{\mathrm{R}}($ minor $)$ $=9.8 \mathrm{~min}] .{ }^{1} \mathrm{H} \mathrm{NMR}\left(400 \mathrm{MHz}, \mathrm{CDCl}_{3}\right) \delta 7.48(\mathrm{~d}, J=8.2 \mathrm{~Hz}, 2 \mathrm{H}), 7.44-7.40(\mathrm{~m}, 1 \mathrm{H})$, $7.36-7.30$ (m, 1H), $7.26-7.17(\mathrm{~m}, 4 \mathrm{H}), 7.13$ (tdd, $J=8.2,3.9,1.3 \mathrm{~Hz}, 2 \mathrm{H}), 6.98$ (qd, 
$J=7.7,0.9 \mathrm{~Hz}, 2 \mathrm{H}), 6.72-6.64(\mathrm{~m}, 1 \mathrm{H}), 3.37-3.24(\mathrm{~m}, 2 \mathrm{H}), 2.98(\mathrm{~d}, J=18.6 \mathrm{~Hz}$, $1 \mathrm{H}), 2.56-2.46(\mathrm{~m}, 1 \mathrm{H}), 2.40(\mathrm{~s}, 3 \mathrm{H}), 1.65(\mathrm{~d}, J=0.9 \mathrm{~Hz}, 18 \mathrm{H}) .{ }^{13} \mathrm{C}$ NMR $(101 \mathrm{MHz}$, $\left.\mathrm{CDCl}_{3}\right) \delta 198.8,175.0,174.9,148.5,141.6,139.2,139.1,139.0,136.7,136.3,130.5$, $129.5,129.3,129.2,128.1,127.2,126.9,125.0,124.1,122.9,122.8,114.4,84.6,84.6$, $50.2,49.2,32.1,28.9,28.1,19.7$. HRMS (ESI) $\mathrm{m} / \mathrm{z}$ calcd for $\mathrm{C}_{38} \mathrm{H}_{38} \mathrm{~N}_{2} \mathrm{O}_{7}[\mathrm{M}+\mathrm{Na}]^{+}=$ 657.2571, found: 657.2576 .
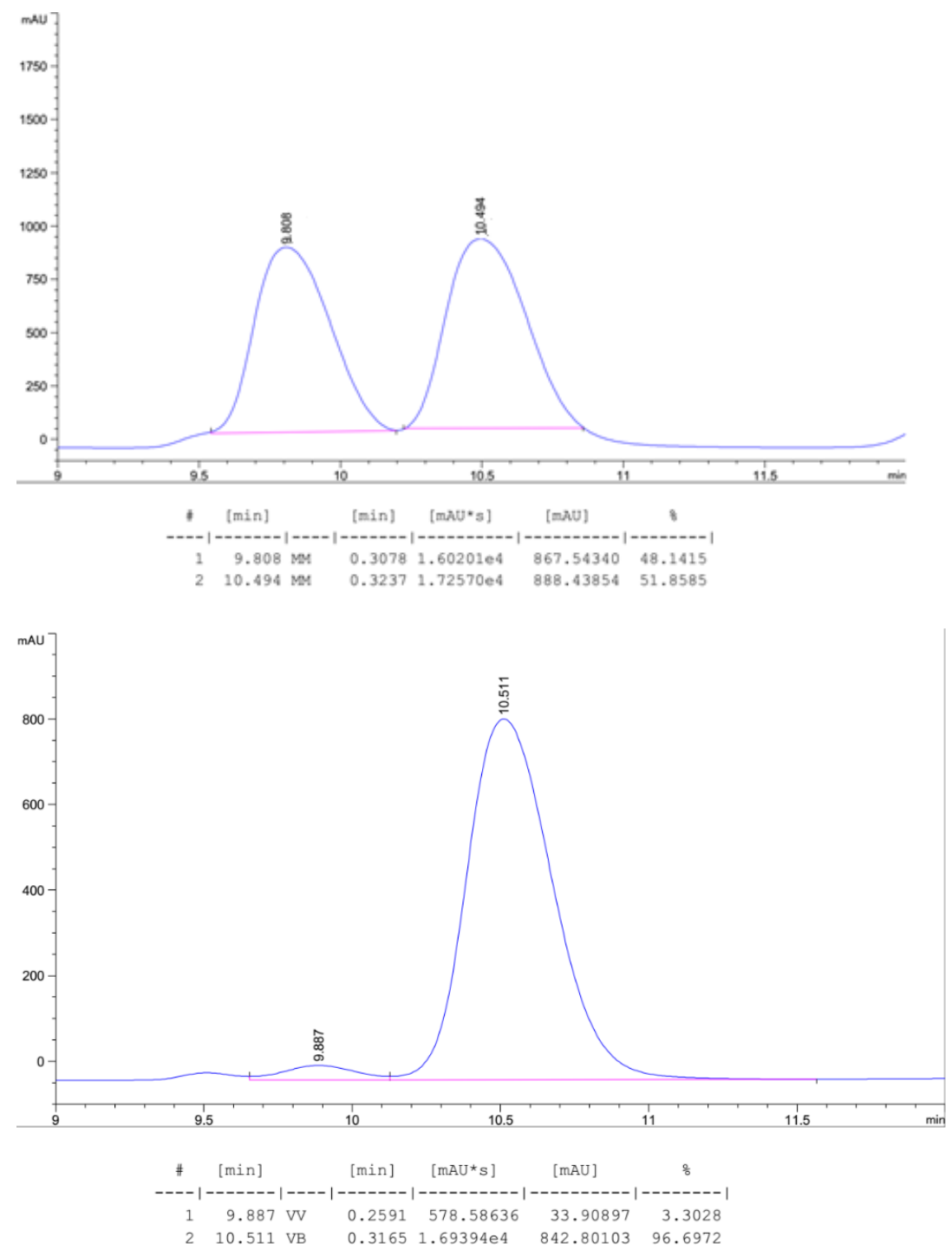

Di-tert-butyl (2'S,3S)-4'-(4-methylbenzoyl)-2,2"-dioxodispiro[indoline-3,1'cyclohexane-2',3"-indolin]-4'-ene-1,1"-dicarboxylate 5p

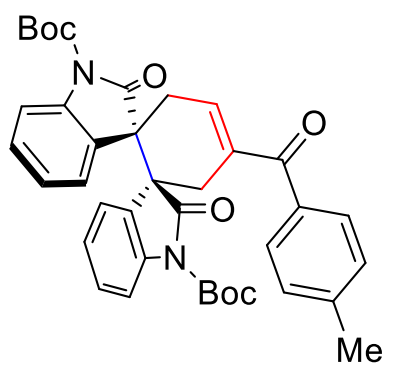

According to general [4 +2] annulation procedure, 5p was obtained in 97\% yield (61.6 $\mathrm{mg}$ ) as a deep red solid and $95 \%$ ee. $[\alpha]_{\mathrm{D}}^{25}=-213.7\left(\mathrm{c} 1.0, \mathrm{CHCl}_{3}\right)$. The ee was 
determined by chiral stationary phase HPLC analysis [Daicel Chiralpak IA, isopropanol $/$ hexane $=5 / 95,0.8 \mathrm{~mL} / \mathrm{min}, \lambda=254 \mathrm{~nm}, \mathrm{t}_{\mathrm{R}}$ (major) $=12.6 \mathrm{~min}, \mathrm{t}_{\mathrm{R}}$ (minor) $=13.3 \mathrm{~min}] .{ }^{1} \mathrm{H}$ NMR $\left(500 \mathrm{MHz}, \mathrm{CDCl}_{3}\right) \delta 7.83-7.69(\mathrm{~m}, 2 \mathrm{H}), 7.52$ (t, $J=7.9 \mathrm{~Hz}$, $2 \mathrm{H}), 7.29-7.22(\mathrm{~m}, 4 \mathrm{H}), 7.16(\mathrm{t}, J=7.3 \mathrm{~Hz}, 2 \mathrm{H}), 7.01(\mathrm{t}, J=7.0 \mathrm{~Hz}, 2 \mathrm{H}), 6.78(\mathrm{~s}, 1 \mathrm{H})$, $3.37(\mathrm{t}, J=19.3 \mathrm{~Hz}, 2 \mathrm{H}), 2.96(\mathrm{~d}, J=18.4 \mathrm{~Hz}, 1 \mathrm{H}), 2.62(\mathrm{~s}, 1 \mathrm{H}), 2.45(\mathrm{~d}, J=6.2 \mathrm{~Hz}$, $3 \mathrm{H}), 1.71-1.67(\mathrm{~m}, 18 \mathrm{H}) .{ }^{13} \mathrm{C} \mathrm{NMR}\left(126 \mathrm{MHz}, \mathrm{CDCl}_{3}\right) \delta 196.6,175.0,148.6,142.2$, 139.2, 138.6, 135.6, 135.4, 129.8, 129.3, 129.2, 128.7, 127.0, 124.1, 122.9, 122.8, 114.5, 84.6, 84.6, 50.2, 49.7, 31.8, 29.8, 28.1, 21.5.HRMS (ESI) m/z calcd for $\mathrm{C}_{38} \mathrm{H}_{38} \mathrm{~N}_{2} \mathrm{O}_{7}[\mathrm{M}$ $+\mathrm{Na}]^{+}=657.2571$, found: 657.2576 .
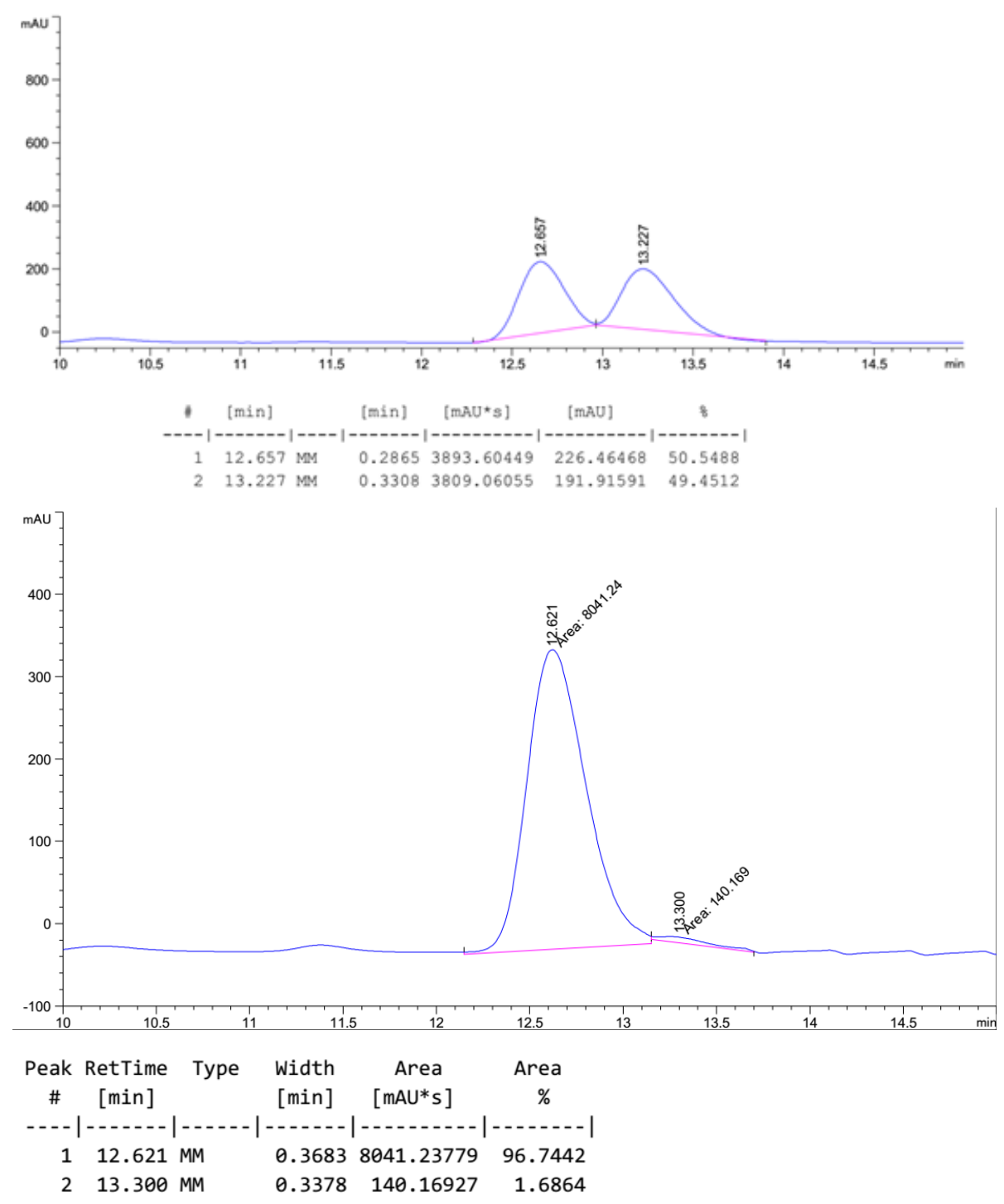

Di-tert-butyl (2'S,3S)-4'-(cyclohexanecarbonyl)-2,2"-dioxodispiro[indoline-3,1'cyclohexane-2',3"-indolin]-4'-ene-1,1"-dicarboxylate $\mathbf{5 q}$ 


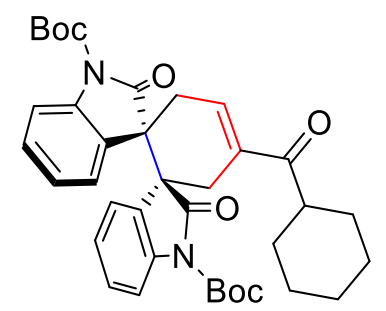

According to general [4+2] annulation procedure, 5q was obtained in 98\% yield (61.4 $\mathrm{mg}$ ) as a deep red solid and $91 \%$ ee. $[\alpha]_{\mathrm{D}}^{25}=-230.7\left(\mathrm{c} 1.0, \mathrm{CHCl}_{3}\right)$. The ee was determined by chiral stationary phase HPLC analysis [Daicel Chiralpak IA, isopropanol $/$ hexane $=5 / 95,0.8 \mathrm{~mL} / \mathrm{min}, \lambda=254 \mathrm{~nm}, \mathrm{t}_{\mathrm{R}}$ (major) $=7.9 \mathrm{~min}, \mathrm{t}_{\mathrm{R}}$ (minor) $=9.2 \mathrm{~min}] .{ }^{1} \mathrm{H}$ NMR $\left(400 \mathrm{MHz}, \mathrm{CDCl}_{3}\right) \delta 7.48(\mathrm{t}, \mathrm{J}=8.3 \mathrm{~Hz}, 2 \mathrm{H}), 7.21-7.08(\mathrm{~m}, 5 \mathrm{H})$, 6.97 (tdd, J = 7.6, 4.5, 1.0 Hz, 2H), $3.36(\mathrm{~d}, \mathrm{~J}=19.4 \mathrm{~Hz}, 1 \mathrm{H}), 3.18-2.99$ (m, 2H), 2.79 $(\mathrm{d}, \mathrm{J}=18.7 \mathrm{~Hz}, 1 \mathrm{H}), 2.58(\mathrm{dd}, \mathrm{J}=19.7,5.0 \mathrm{~Hz}, 1 \mathrm{H}), 1.91-1.79(\mathrm{~m}, 4 \mathrm{H}), 1.64(\mathrm{~d}, \mathrm{~J}=$ $2.3 \mathrm{~Hz}, 18 \mathrm{H}), 1.49-1.41(\mathrm{~m}, 2 \mathrm{H}), 1.35-1.25(\mathrm{~m}, 4 \mathrm{H}){ }^{13} \mathrm{C} \mathrm{NMR}\left(101 \mathrm{MHz}, \mathrm{CDCl}_{3}\right) \delta$ 196.9, 174.9, 174.9, 148.6, 148.6, 139.4, 139.2, 139.0, 138.5, 137.9, 135.4, 132.3, 129.7, 129.3, 129.2, 129.1, 127.8, 127.2, 127.0, 126.8, 124.4, 124.1, 124.0, 123.1, 122.9, 122.8, $115.5,114.4,84.6,84.5,50.2,49.7,47.3,31.9,29.6,28.1,28.0,21.3$. HRMS (ESI) m/z calcd for $\mathrm{C}_{38} \mathrm{H}_{38} \mathrm{~N}_{2} \mathrm{O}_{7}[\mathrm{M}+\mathrm{Na}]^{+}=649.2884$, found: 649.2886.

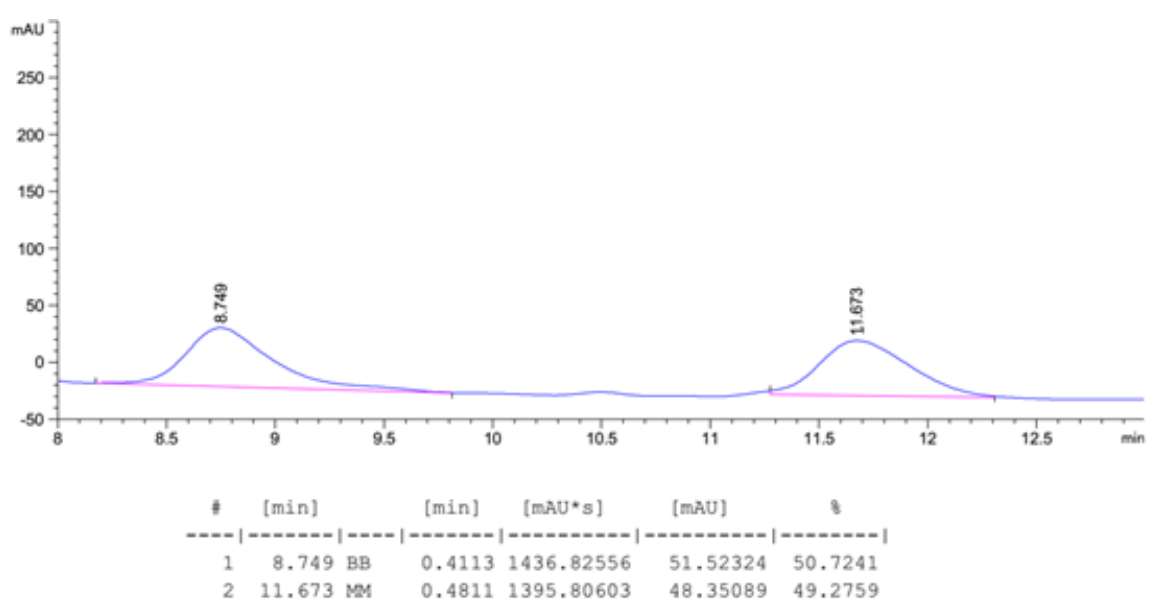




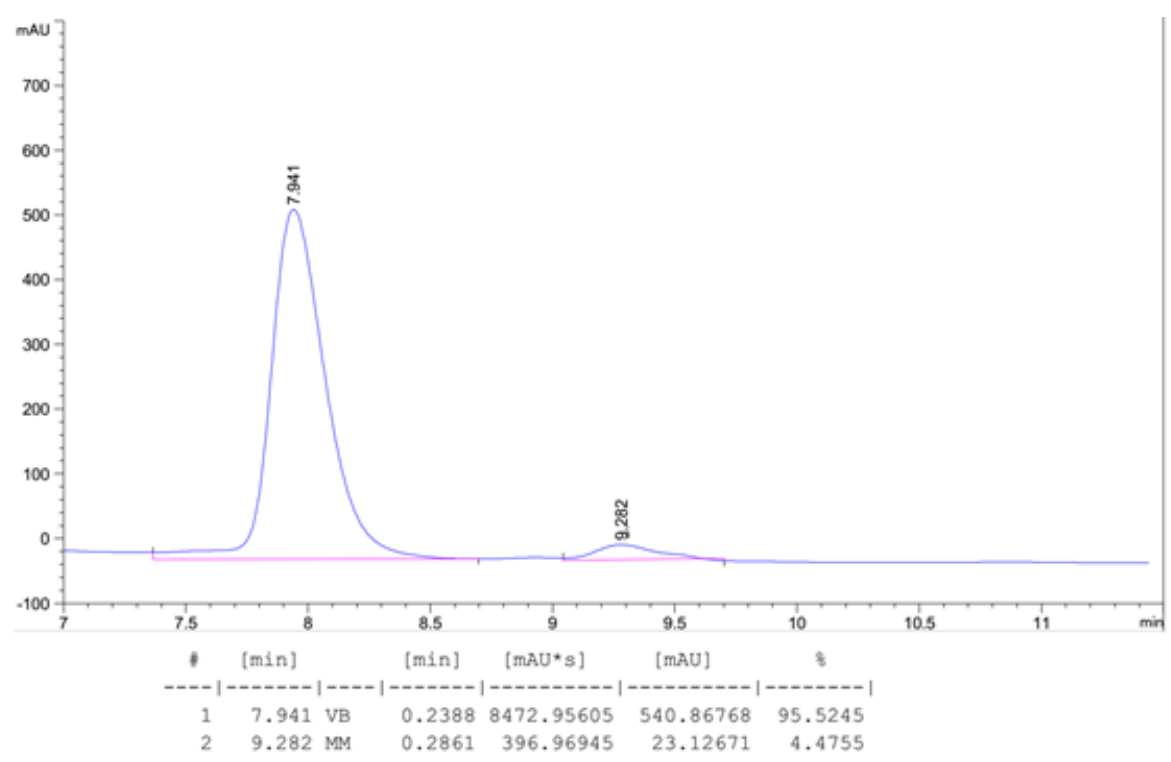

Di-tert-butyl (3S,3'S)-3-(2,3-dioxo-3-phenylpropyl)-2,2'-dioxo-3'-(2-oxoethyl)-[3,3'biindoline]-1,1'-dicarboxylate 6<smiles>CC(C)(C)OC(=O)N1C(=O)[C@](CC(=O)C(=O)c2ccccc2)(C(=O)C(=O)c2ccccc2)c2ccccc21</smiles>

6 was obtained as a light-yellow oil. ${ }^{1} \mathrm{H}$ NMR $\left(500 \mathrm{MHz}, \mathrm{CDCl}_{3}\right) \delta 9.46(\mathrm{~d}, J=0.7 \mathrm{~Hz}$, $1 \mathrm{H}), 7.71-7.66(\mathrm{~m}, 2 \mathrm{H}), 7.58(\mathrm{~d}, J=7.5 \mathrm{~Hz}, 1 \mathrm{H}), 7.47-7.43(\mathrm{~m}, 2 \mathrm{H}), 7.39$ (dd, $J=$ 8.1, $7.6 \mathrm{~Hz}, 2 \mathrm{H}), 7.13(\mathrm{t}, J=7.9 \mathrm{~Hz}, 4 \mathrm{H}), 6.97-6.90(\mathrm{~m}, 2 \mathrm{H}), 4.64(\mathrm{~d}, J=18.6 \mathrm{~Hz}$, $1 \mathrm{H}), 4.14(\mathrm{~d}, J=7.1 \mathrm{~Hz}, 2 \mathrm{H}), 3.79(\mathrm{~d}, J=18.6 \mathrm{~Hz}, 1 \mathrm{H}), 3.48(\mathrm{~d}, J=18.5 \mathrm{~Hz}, 1 \mathrm{H}), 1.68$ (s, 9H), $1.66(\mathrm{~s}, 9 \mathrm{H}) .{ }^{13} \mathrm{C}$ NMR $\left(126 \mathrm{MHz}, \mathrm{CDCl}_{3}\right) \delta 198.4,196.8,190.0,175.3,174.9$, $148.3,140.1,139.9,134.7,131.2,130.2,129.5,129.4,128.7,124.8,124.7,123.6,123.6$, $122.3,122.2,114.7,114.6,84.5,52.9,52.4,44.1,39.3,28.1,28.1$.

Di-tert-butyl (3S,3'S)-3-(2-methoxy-2-oxoethyl)-2,2'-dioxo-3'-(2-oxoethyl)-[3,3'biindoline]-1,1'-dicarboxylate 7<smiles>COC(=O)C[C@]1(C(=O)O)c2ccccc2N(C(=O)OC(C)(C)C)C(=O)[C@]1(CC(=O)O)C(=O)OC(C)(C)C</smiles>

7 was obtained as a white solid. ${ }^{1} \mathrm{H}$ NMR $\left(500 \mathrm{MHz}, \mathrm{CDCl}_{3}\right) \delta 9.44(\mathrm{~s}, 1 \mathrm{H}), 7.42(\mathrm{t}, J$ $=8.0 \mathrm{~Hz}, 2 \mathrm{H}), 7.17-7.06(\mathrm{~m}, 4 \mathrm{H}), 6.93(\mathrm{dd}, J=17.4,7.5 \mathrm{~Hz}, 2 \mathrm{H}), 4.15(\mathrm{~d}, J=18.4$ $\mathrm{Hz}, 1 \mathrm{H}), 3.84(\mathrm{~d}, J=16.5 \mathrm{~Hz}, 1 \mathrm{H}), 3.43-3.31(\mathrm{~m}, 5 \mathrm{H}), 1.66(\mathrm{~d}, J=1.7 \mathrm{~Hz}, 18 \mathrm{H}) .{ }^{13} \mathrm{C}$ NMR $\left(126 \mathrm{MHz} \mathrm{CDCl}_{3}\right) \delta 196.9,175.4,174.9,169.9,148.3,139.9,139.8,129.4,129.3$, 
$125.0,124.9,123.6,123.5,122.4,122.3,114.6,114.5,84.4,84.4,53.5,52.3,51.9,43.9$, $34.5,28.1$.

Dimethyl 2,2'-((3S,3'S)-1,1'-dimethyl-2,2'-dioxo-[3,3'-biindoline]-3,3'-diyl)diacetate 8<smiles>COC(=O)C[C@@]1(C(=O)C(=O)OC)C(=O)N(C)c2ccccc21</smiles>

8 was obtained as a white solid. ${ }^{1} \mathrm{H}$ NMR $\left(500 \mathrm{MHz}, \mathrm{CDCl}_{3}\right) \delta 7.08-7.01(\mathrm{~m}, 4 \mathrm{H})$, $6.83(\mathrm{td}, J=7.6,0.9 \mathrm{~Hz}, 2 \mathrm{H}), 6.42(\mathrm{~d}, J=7.7 \mathrm{~Hz}, 2 \mathrm{H}), 4.06(\mathrm{~d}, J=16.2 \mathrm{~Hz}, 2 \mathrm{H}), 3.38$ (s, 6H), $3.23(\mathrm{~d}, J=16.2 \mathrm{~Hz}, 2 \mathrm{H}), 3.12(\mathrm{~s}, 6 \mathrm{H}) .{ }^{13} \mathrm{C} \mathrm{NMR}\left(126 \mathrm{MHz}, \mathrm{CDCl}_{3}\right) \delta 176.6$, $170.3,143.9,128.8,126.8,122.7,121.4,107.3,105.0,52.4,51.6,33.8,25.8$.

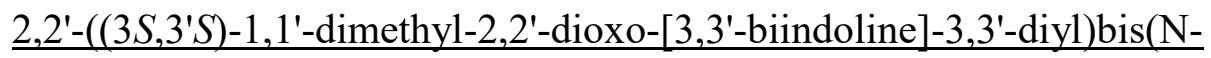
$\underline{\text { methylacetamide) } 9}$<smiles>CNC(=O)C[C@]1(C(=O)C(=O)NC)C(=O)N(C)c2ccccc21</smiles>

9 was obtained as a white solid, ${ }^{1} \mathrm{HNMR}$ was identical as the literature ${ }^{3} .{ }^{1} \mathrm{H}$ NMR (400 MHz, DMSO) $\delta 7.83(\mathrm{~d}, J=4.6 \mathrm{~Hz}, 2 \mathrm{H}), 6.94(\mathrm{td}, J=7.6,1.3 \mathrm{~Hz}, 2 \mathrm{H}), 6.82(\mathrm{dd}, J=$ $7.4,0.9 \mathrm{~Hz}, 2 \mathrm{H}), 6.74(\mathrm{td}, J=7.5,0.8 \mathrm{~Hz}, 2 \mathrm{H}), 6.46(\mathrm{~d}, J=7.7 \mathrm{~Hz}, 2 \mathrm{H}), 3.68(\mathrm{~d}, J=$ $15.1 \mathrm{~Hz}, 2 \mathrm{H}), 2.93(\mathrm{~s}, 6 \mathrm{H}), 2.85(\mathrm{~d}, J=15.1 \mathrm{~Hz}, 2 \mathrm{H}), 2.23(\mathrm{~d}, J=4.5 \mathrm{~Hz}, 6 \mathrm{H}) .{ }^{13} \mathrm{C} \mathrm{NMR}$ (126 MHz, DMSO) $\delta 177.04,169.09,144.42,128.43,127.93,122.53,120.88,107.60$, 52.66, 35.07, 25.96, 25.71.

2-((S)-3-((3aS,8aS)-1,8-dimethyl-2-oxo-2,3,8,8a-tetrahydropyrrolo[2,3-b]indol$\underline{3 \mathrm{a}(1 \mathrm{H}) \text {-yl)-1-methyl-2-oxoindolin-3-yl)-N-methylacetamide } 11}$<smiles>CN1C(=O)C[C@]2(c3ccccc31)c1ccccc1N(C)[C@H]2N(C)C</smiles>

Colorless oil. ${ }^{1} \mathrm{H}$ NMR $\left(400 \mathrm{MHz}, \mathrm{CDCl}_{3}\right) \delta 7.13(\mathrm{td}, J=7.7,1.0 \mathrm{~Hz}, 2 \mathrm{H}), 7.06(\mathrm{~d}, J$ $=7.3 \mathrm{~Hz}, 2 \mathrm{H}), 6.72-6.66(\mathrm{~m}, 2 \mathrm{H}), 6.39(\mathrm{~d}, \mathrm{~J}=7.8 \mathrm{~Hz}, 2 \mathrm{H}), 4.32(\mathrm{~s}, 2 \mathrm{H}), 3.00-2.94$ $(\mathrm{m}, 8 \mathrm{H}), 2.75(\mathrm{~d}, \mathrm{~J}=17.2 \mathrm{~Hz}, 2 \mathrm{H}), 2.65(\mathrm{~s}, 6 \mathrm{H}) .{ }^{13} \mathrm{C} \mathrm{NMR}\left(126 \mathrm{MHz}, \mathrm{CDCl}_{3}\right) \delta$ 
$171.77,149.56,130.36,130.02,124.50,118.91,107.44,87.33,53.99,39.33,34.38$, 28.36 .

\section{(-)-Folicanthine}

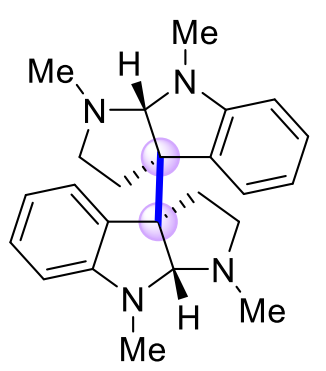

(-)-Folicanthine

(-)-Folicanthine was obtained as a white solide, the product is light sensitive easily decomposed in $\mathrm{CDCl}_{3}$ solution. ${ }^{1} \mathrm{HNMR}$ and ${ }^{13} \mathrm{CNMR}$ was identical as the literature ${ }^{4}$. $[\mathrm{a}]^{25} \mathrm{D}=-19.5(\mathrm{c} 0.2, \mathrm{MeOH}) .{ }^{1} \mathrm{H}$ NMR $\left(400 \mathrm{MHz}, \mathrm{CDCl}_{3}\right) \delta 6.99(\mathrm{t}, \mathrm{J}=7.2 \mathrm{~Hz}, 2 \mathrm{H})$, $6.94(\mathrm{~m}, 2 \mathrm{H}), 6.52(\mathrm{t}, \mathrm{J}=6.8 \mathrm{~Hz}, 2 \mathrm{H}), 6.28(\mathrm{~d}, \mathrm{~J}=7.6 \mathrm{~Hz}, 2 \mathrm{H}), 4.93$ (brs, 2H), 3.01 (s, $6 \mathrm{H}), 2.65(\mathrm{~m}, 2 \mathrm{H}), 2.50-2.42(\mathrm{~m}, 10 \mathrm{H}), 1.97-2.00(\mathrm{~m}, 2 \mathrm{H}) .{ }^{13} \mathrm{CNMR}\left(126 \mathrm{MHz}, \mathrm{CDCl}_{3}\right)$ $\delta 152.9,132.8,128.1,123.6,116.6,105.8,91.8,62.6,52.6,37.9,35.4,35.2$; HRMS (ESI) $\mathrm{m} / \mathrm{z}$ calcd for $\mathrm{C}_{24} \mathrm{H}_{30} \mathrm{~N}_{4}[\mathrm{M}+\mathrm{Na}]^{+}=397.2363$, found: 397.2368 .

\section{J. X-ray Analysis of 5g}

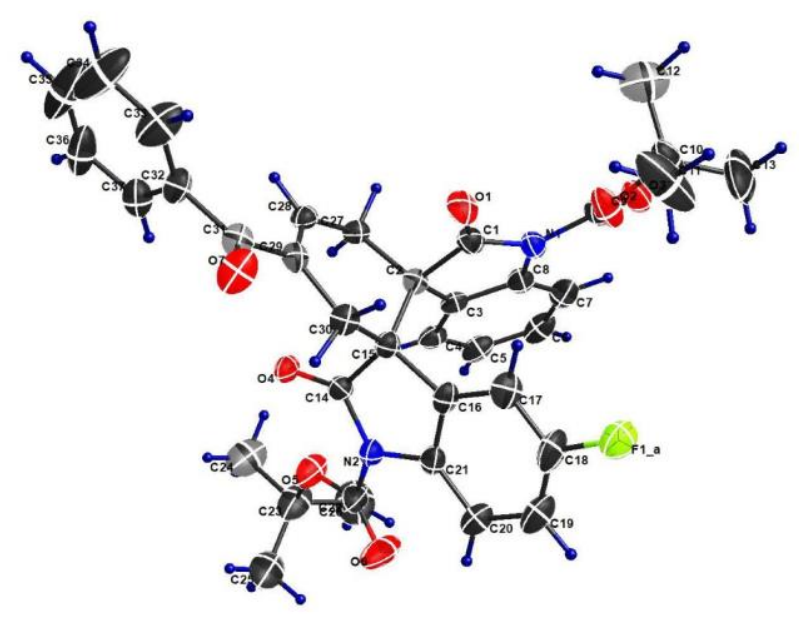




\begin{tabular}{lll}
\hline \multicolumn{3}{l}{ Table S1. Sample and crystal data for 5g. } \\
\hline Identification code & $\mathrm{J} 579$ & \\
Chemical formula & $\mathrm{C}_{37} \mathrm{H}_{35} \mathrm{FN}_{2} \mathrm{O}_{7}$ & \\
Formula weight & $638.67 \mathrm{~g} / \mathrm{mol}$ & \\
Temperature & $100(2) \mathrm{K}$ & \\
Wavelength & $1.54178 \AA$ & \\
Crystal size & $0.222 \times 0.474 \times 0.536 \mathrm{~mm}$ \\
Crystal system & orthorhombic & \\
Space group & $\mathrm{P} 212121$ & \\
Unit cell dimensions & $\mathrm{a}=16.485(3) \AA$ & $\alpha=90^{\circ}$ \\
& $\mathrm{b}=24.296(4) \AA$ & $\beta=90^{\circ}$ \\
& $\mathrm{c}=24.715(4) \AA$ & $\gamma=90^{\circ}$ \\
Volume & $9899 .(3) \AA^{3}$ & \\
Z & 12 & \\
Density (calculated) & $1.286 \mathrm{~g} / \mathrm{cm}^{3}$ & \\
Absorption coefficient & $0.766 \mathrm{~mm}^{-1}$ & \\
F(000) & 4032 & \\
\hline
\end{tabular}

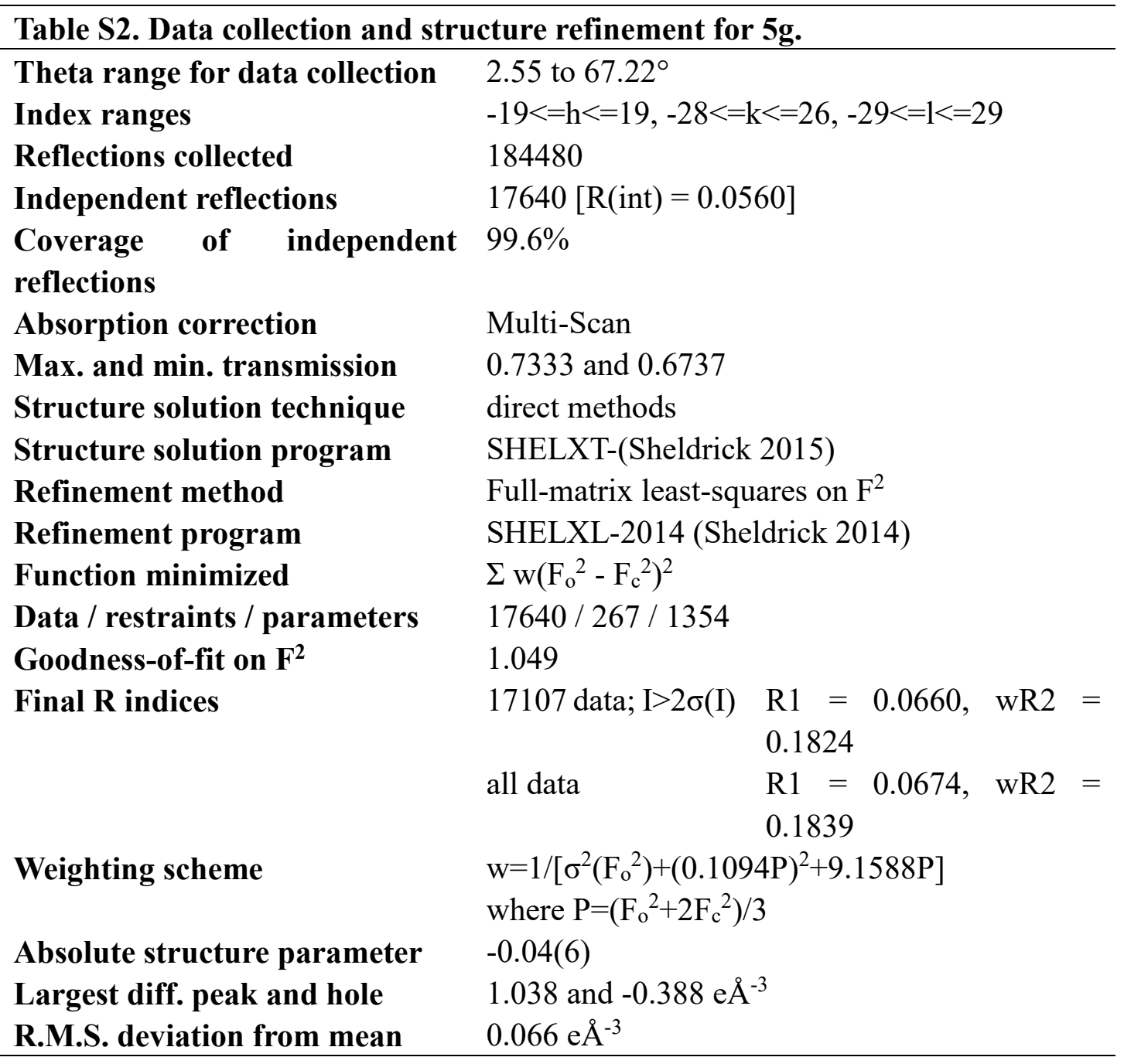


K. ${ }^{1} \mathrm{H}$ and ${ }^{13} \mathrm{C}$ NMR spectra
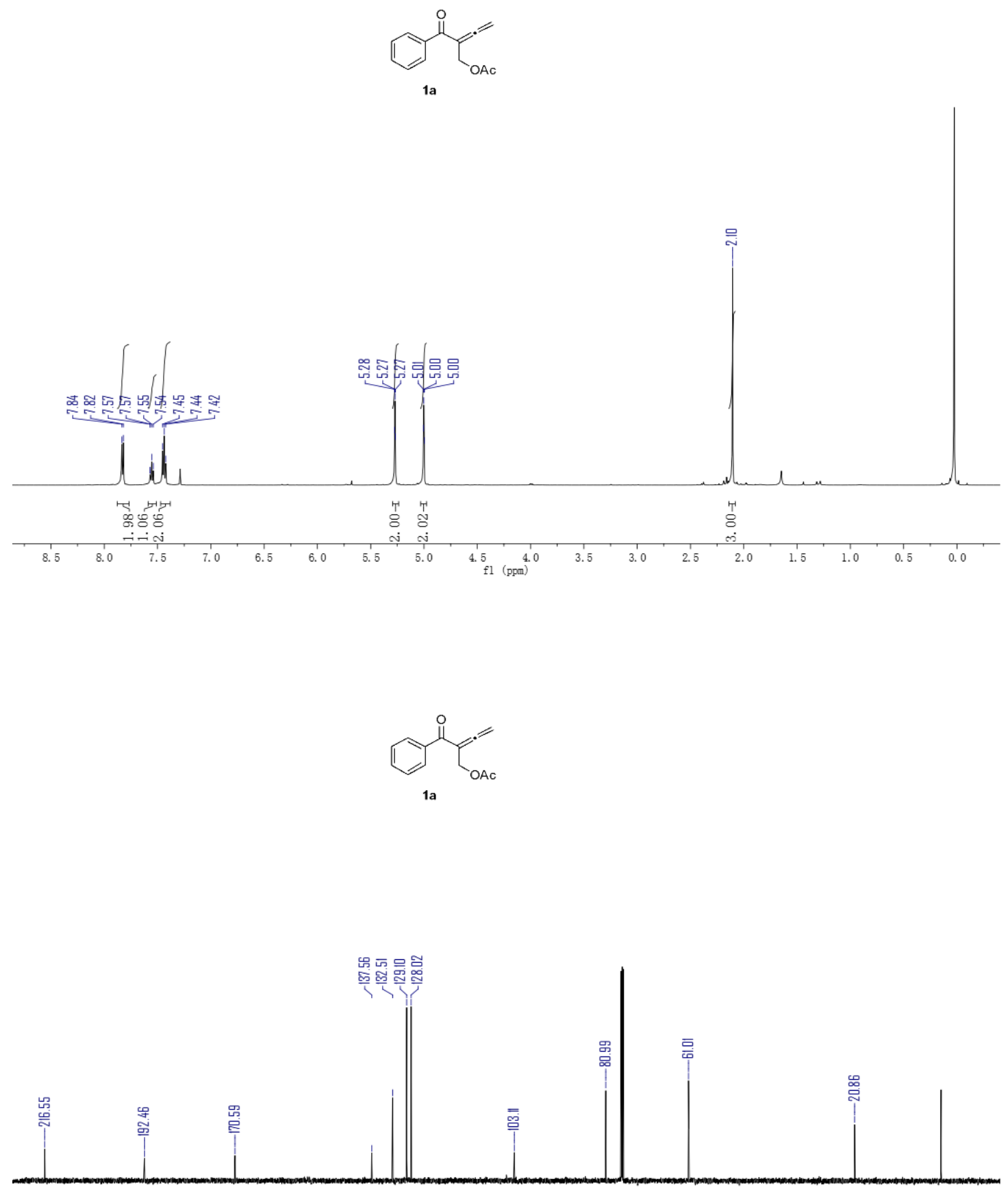

$\begin{array}{rllllllllllll}220 & 210 & 200 & 190 & 180 & 170 & 160 & 150 & 140 & 130 & 120 & 110 & 100 \\ \mathrm{f} 1 & (\mathrm{ppm})\end{array}$ 

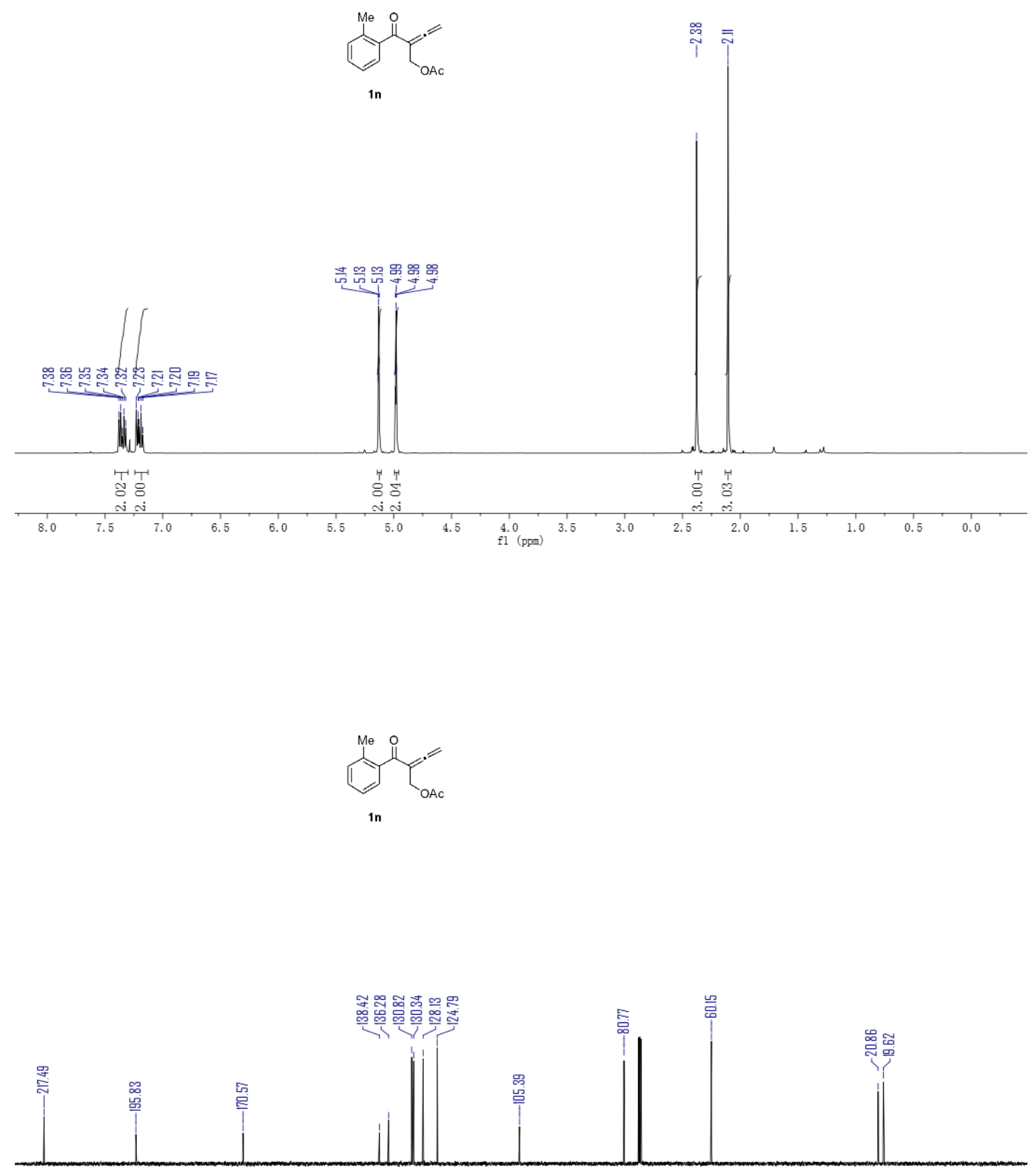

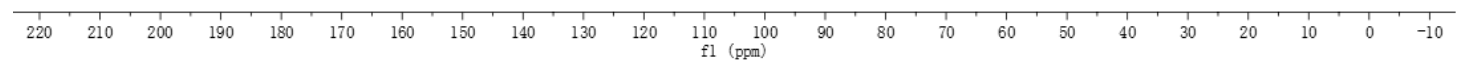



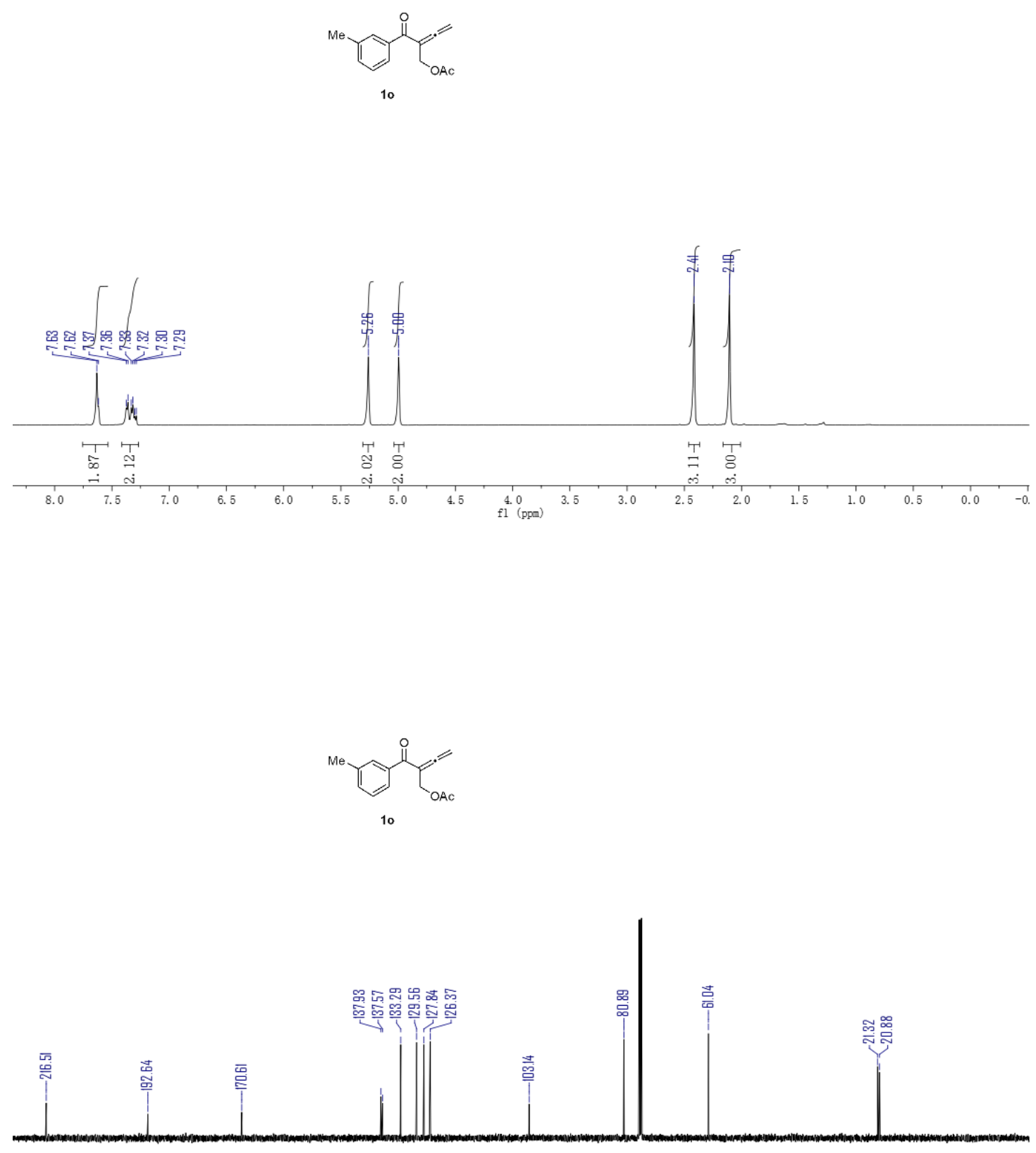

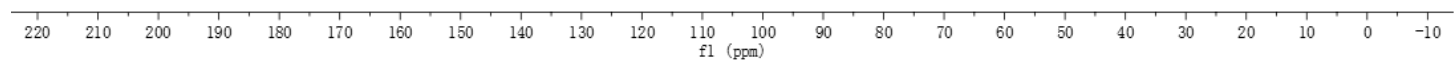



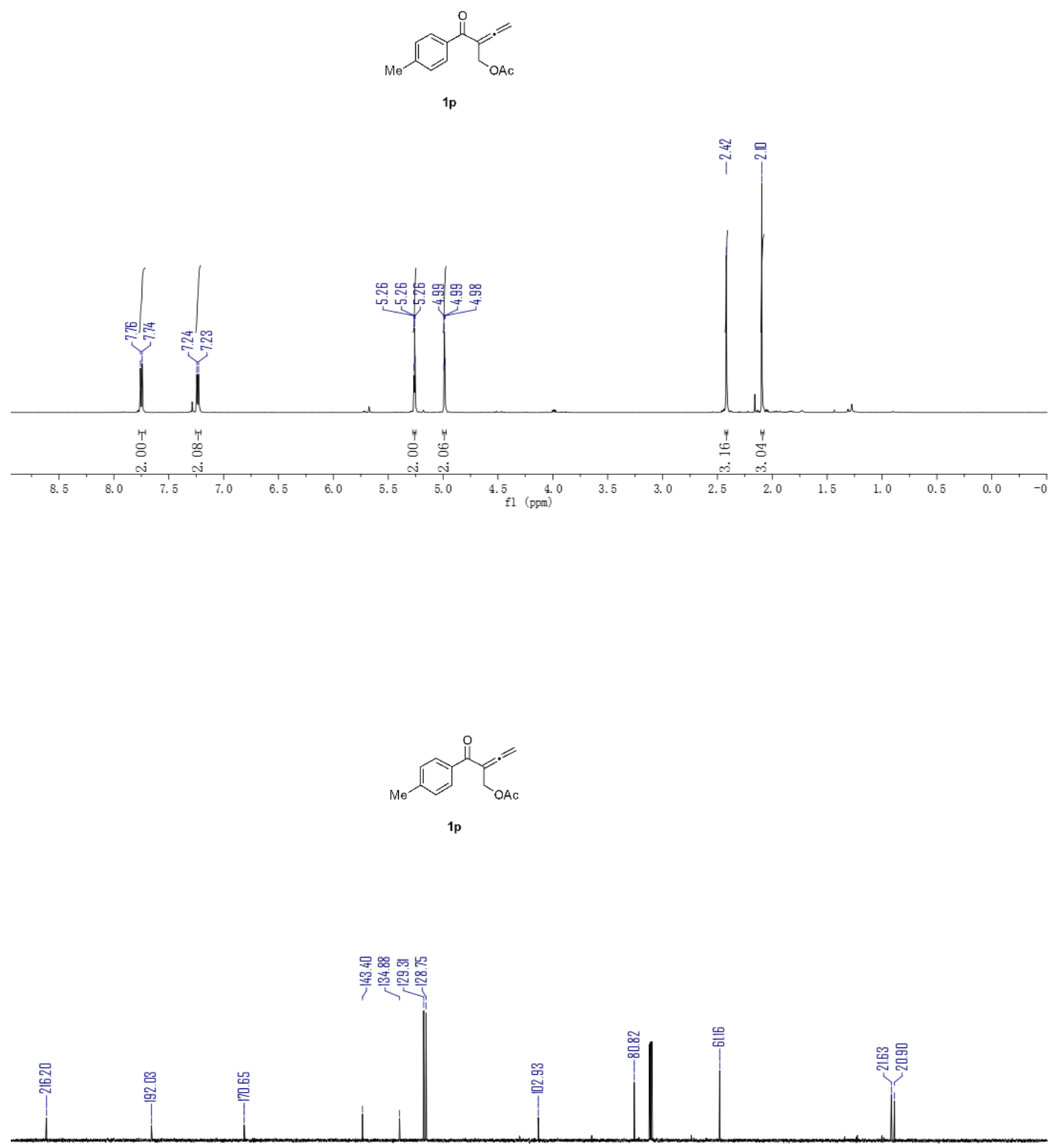

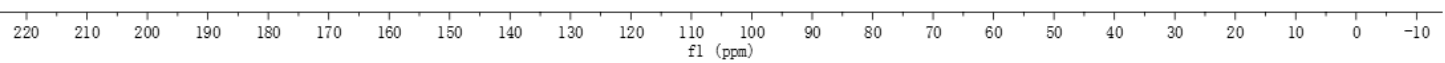




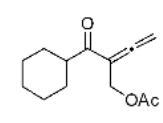

$1 q$
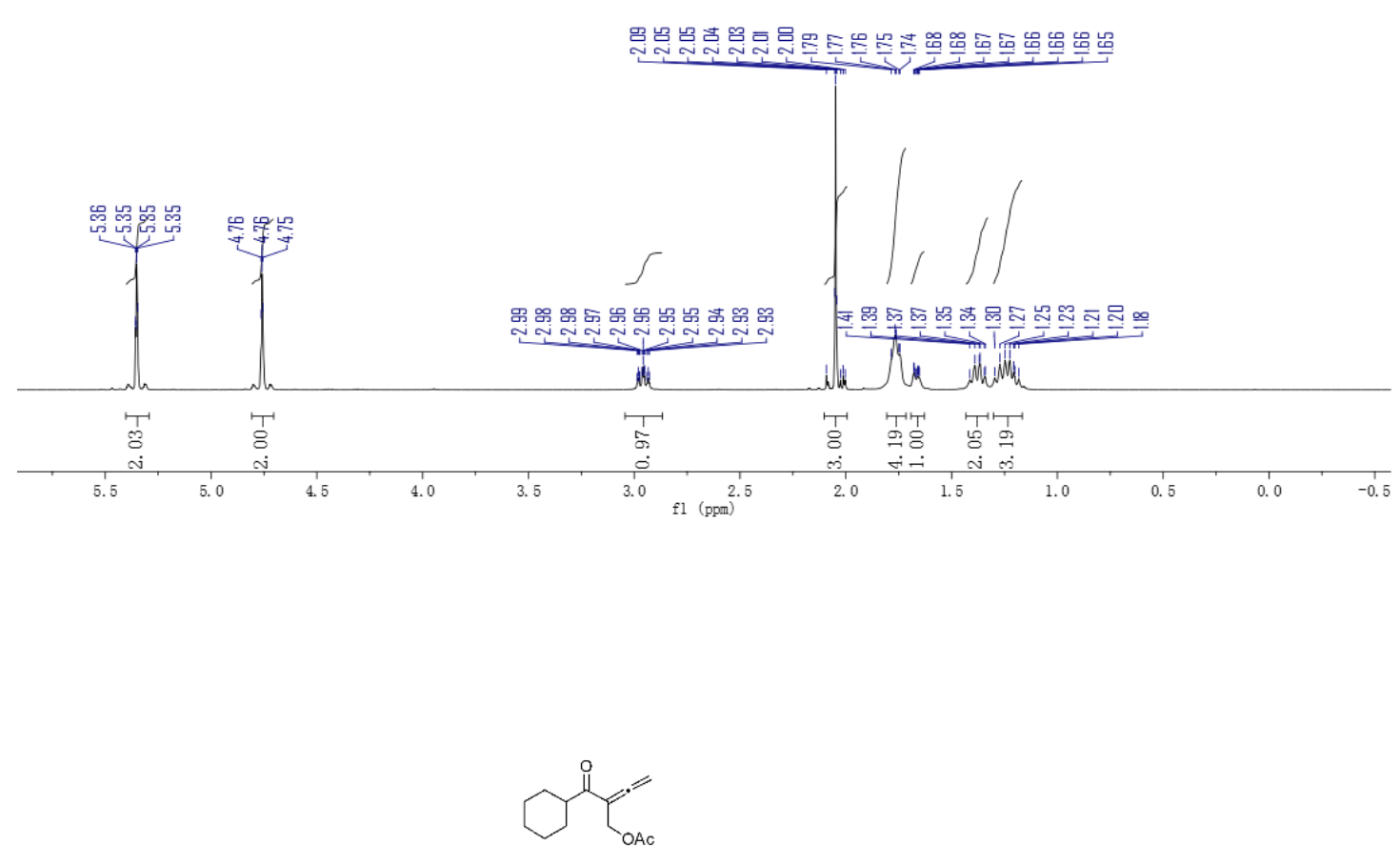

$1 q$

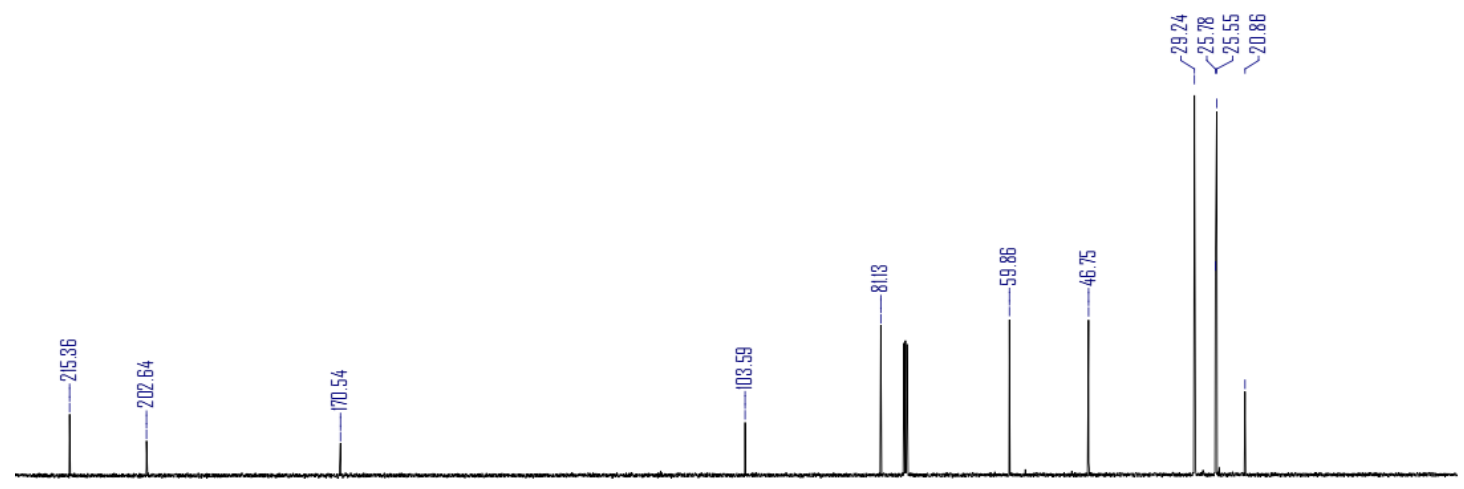

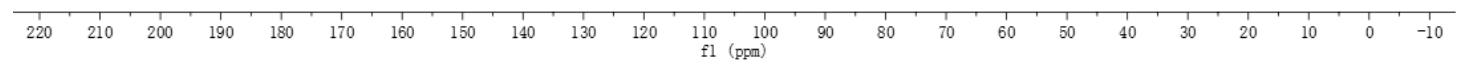




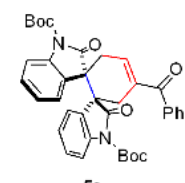

$5 a$

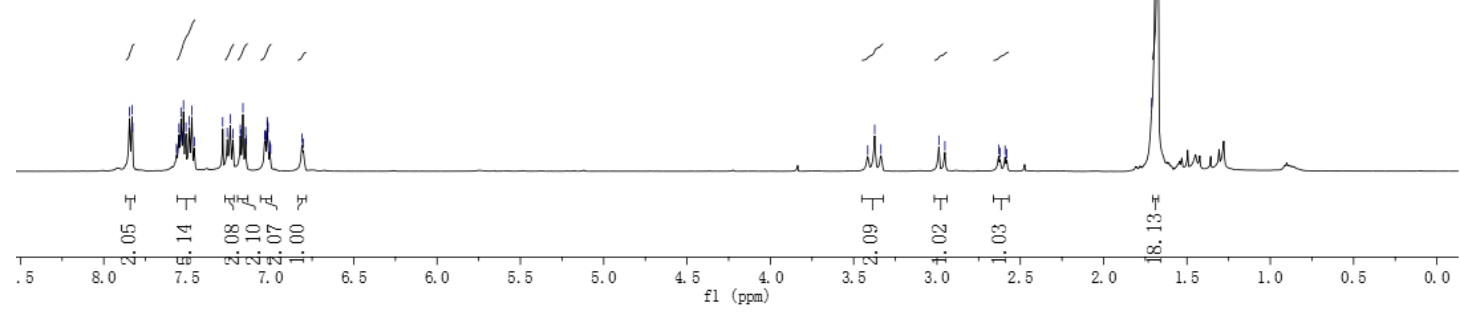

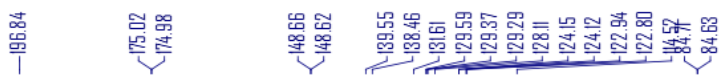

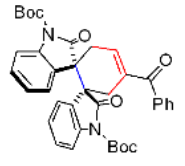

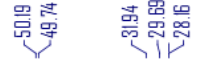

$5 a$

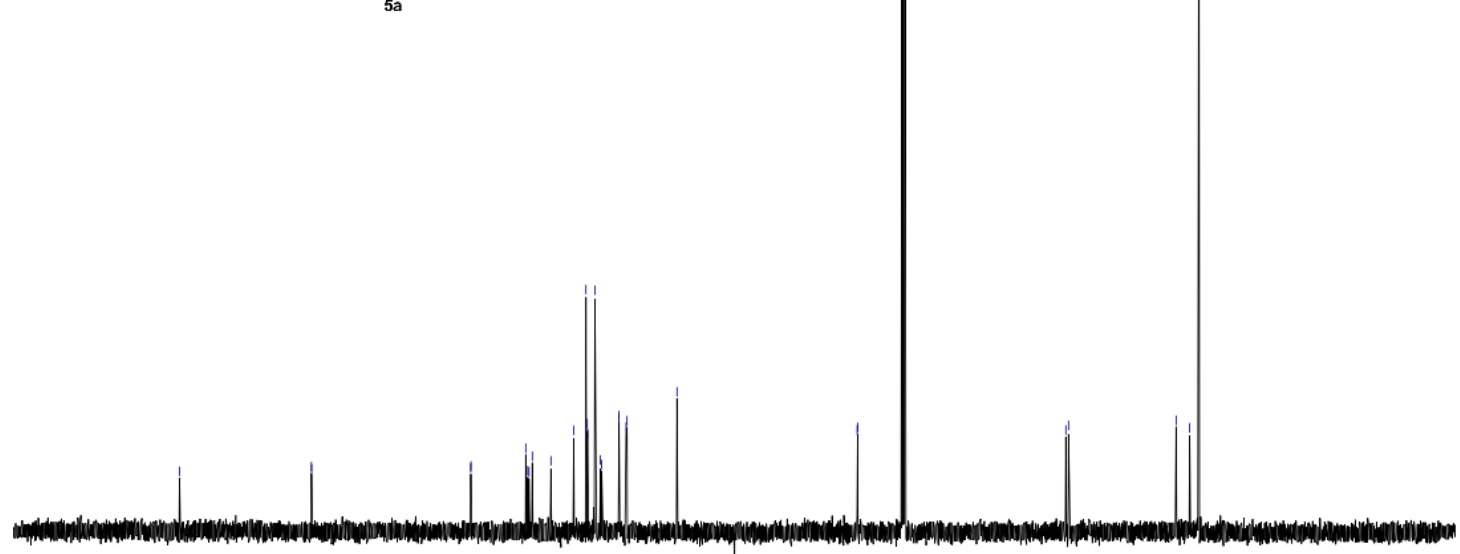

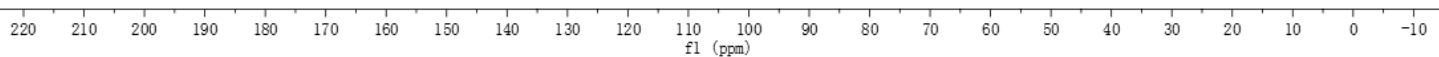




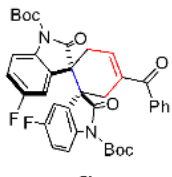

5b

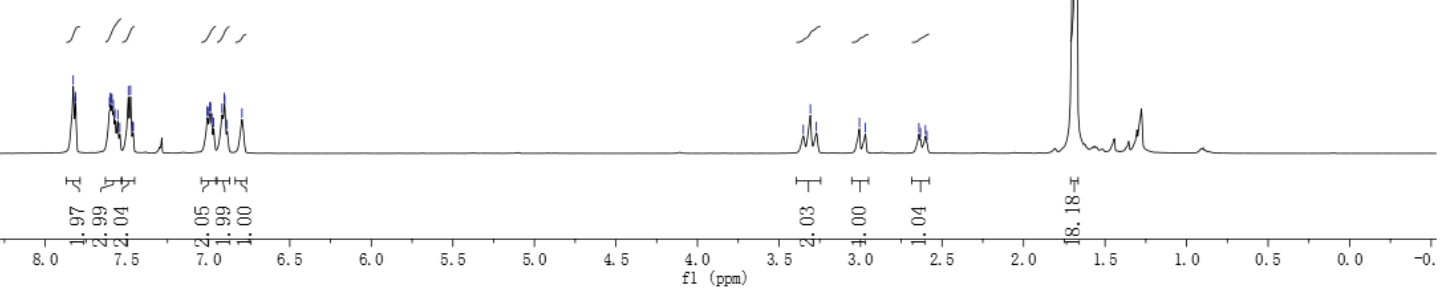

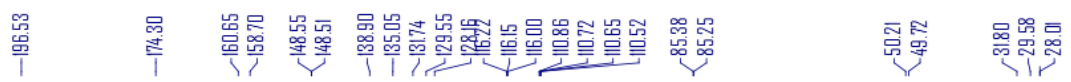
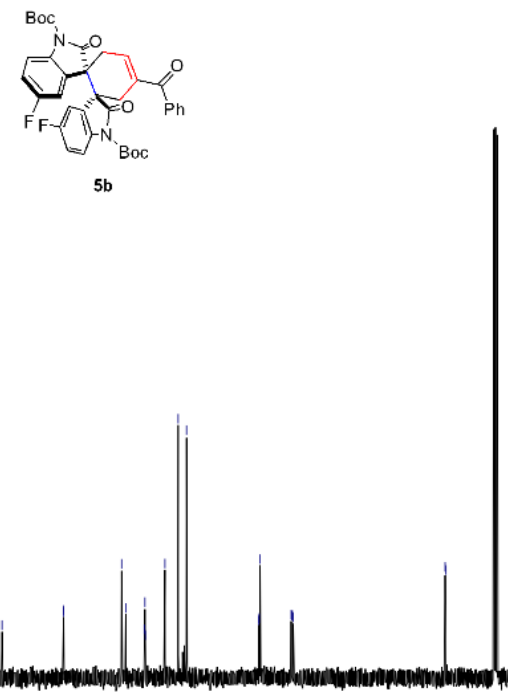

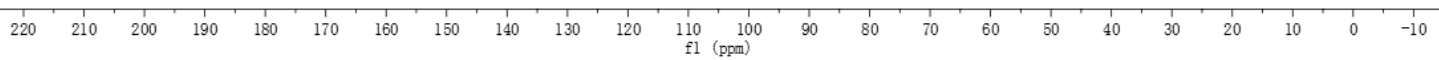



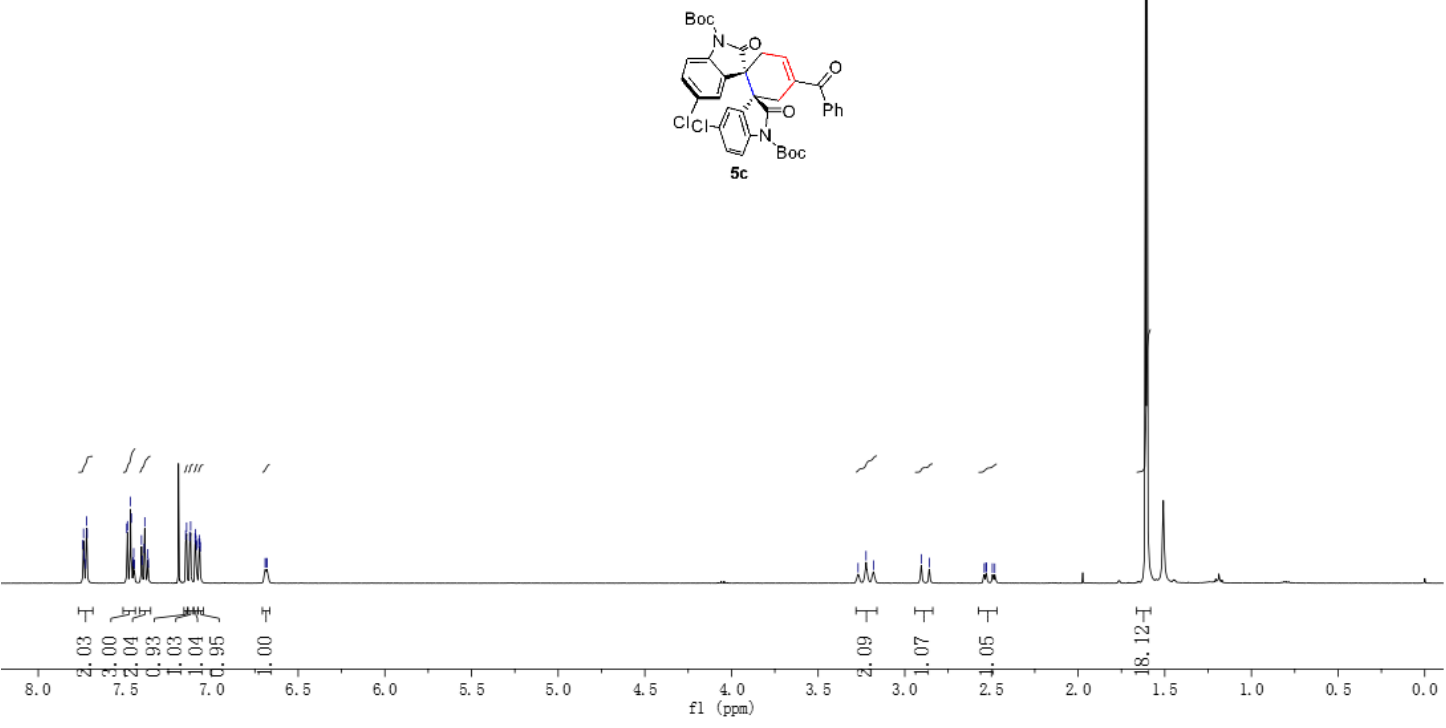

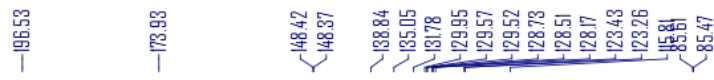
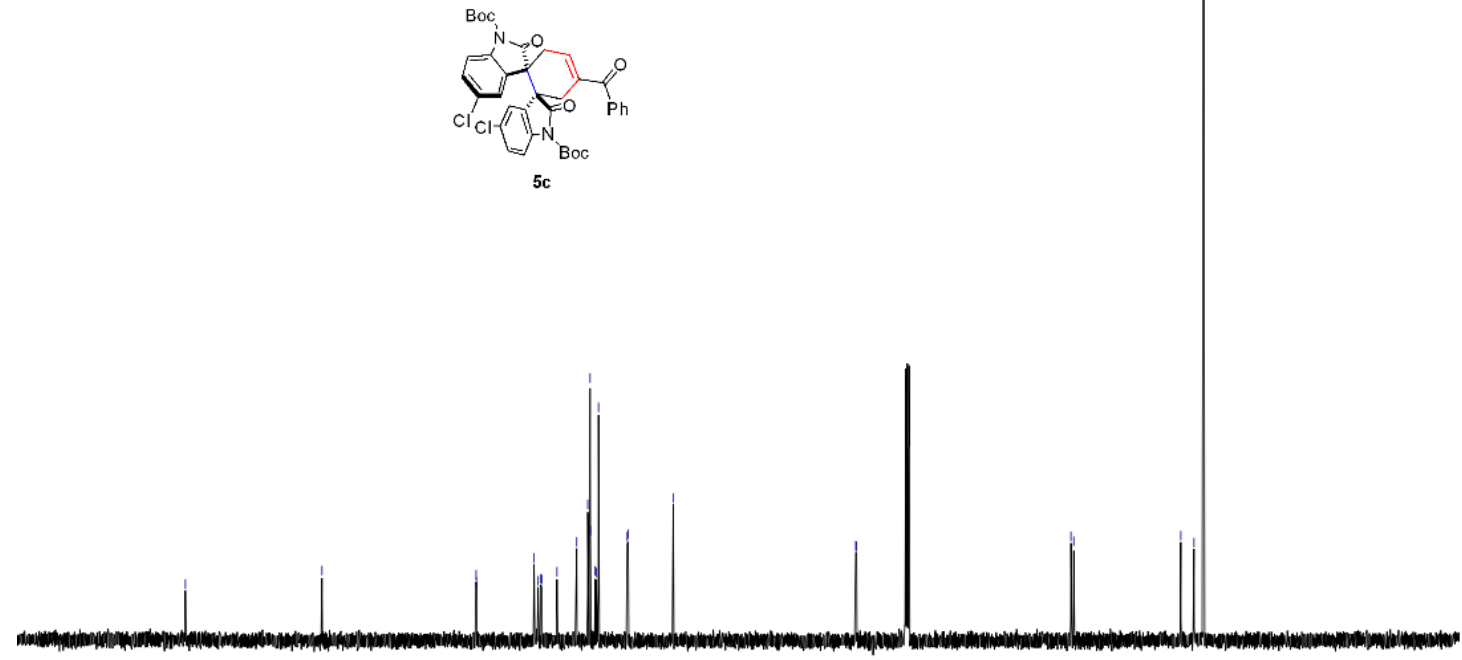

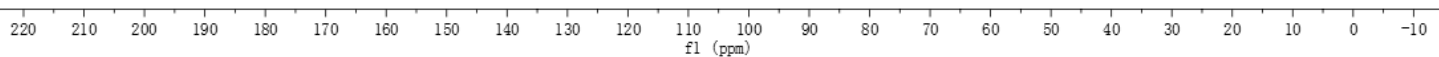



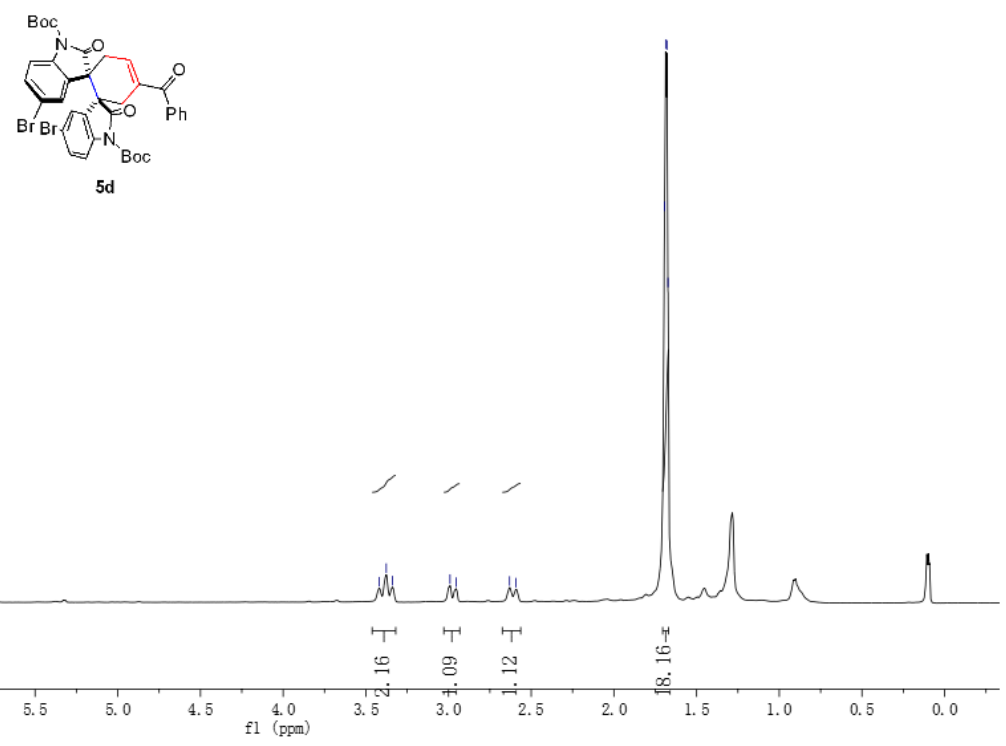

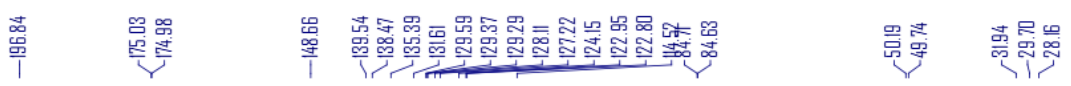
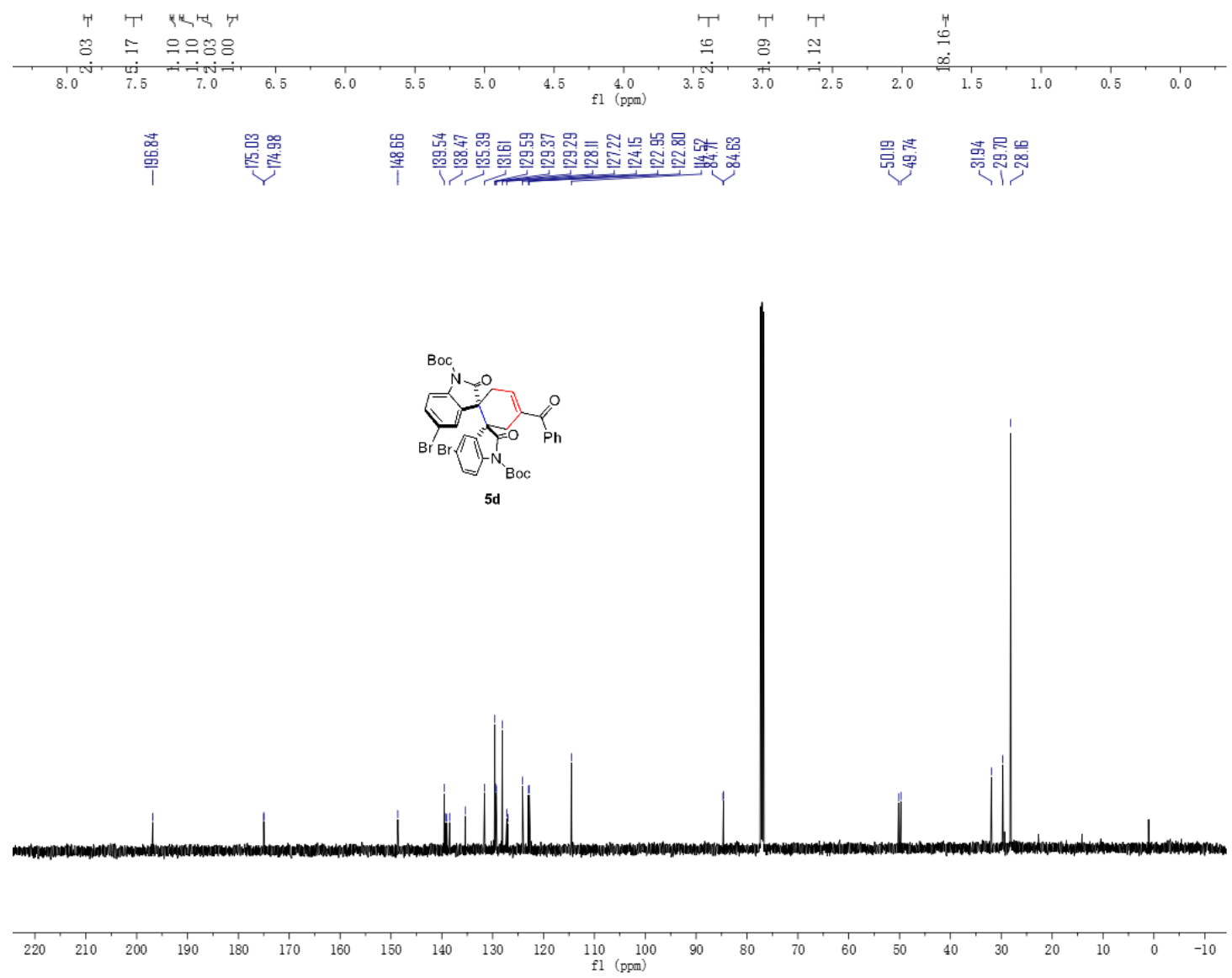


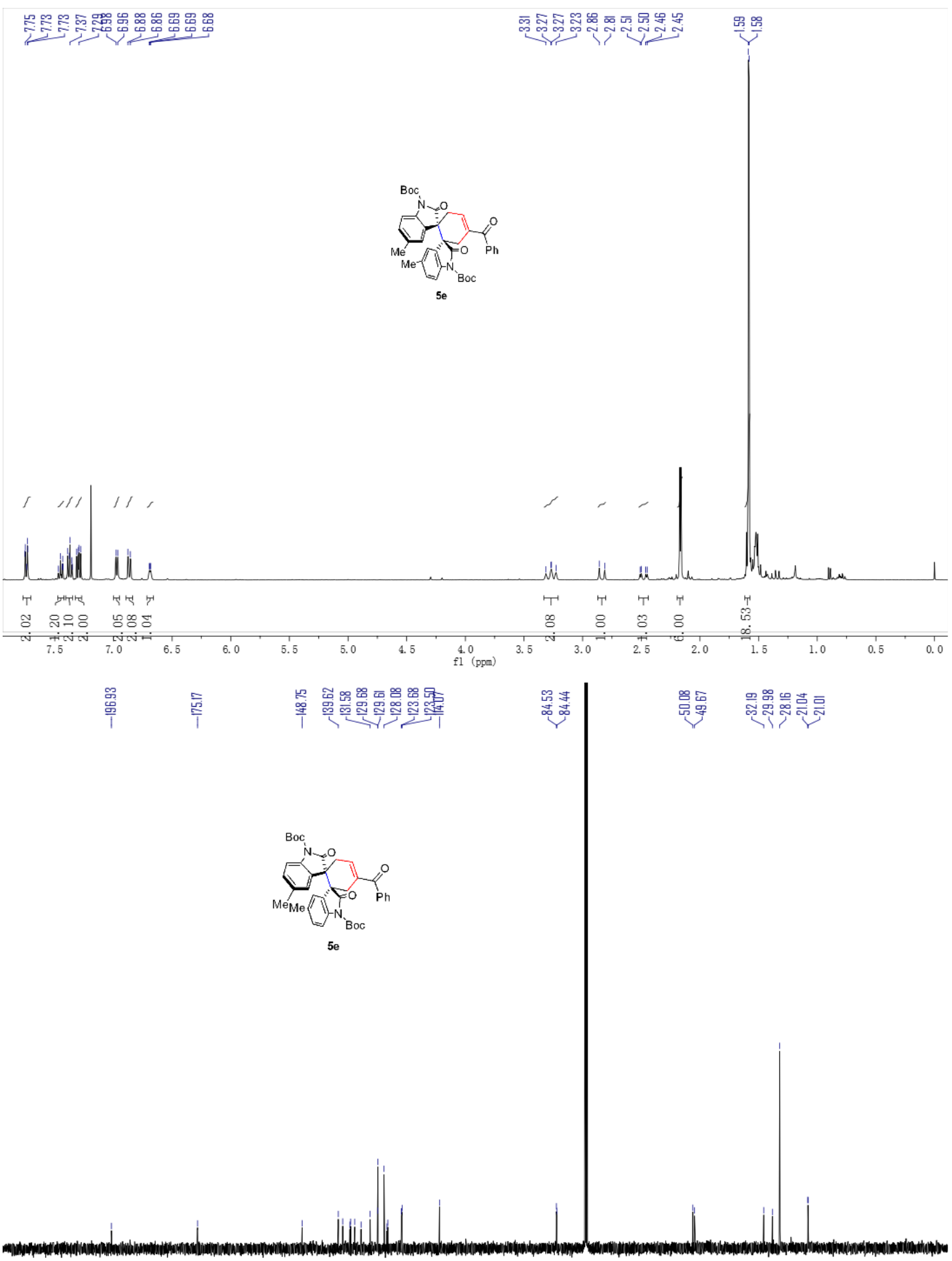

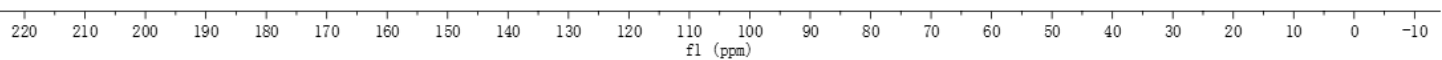



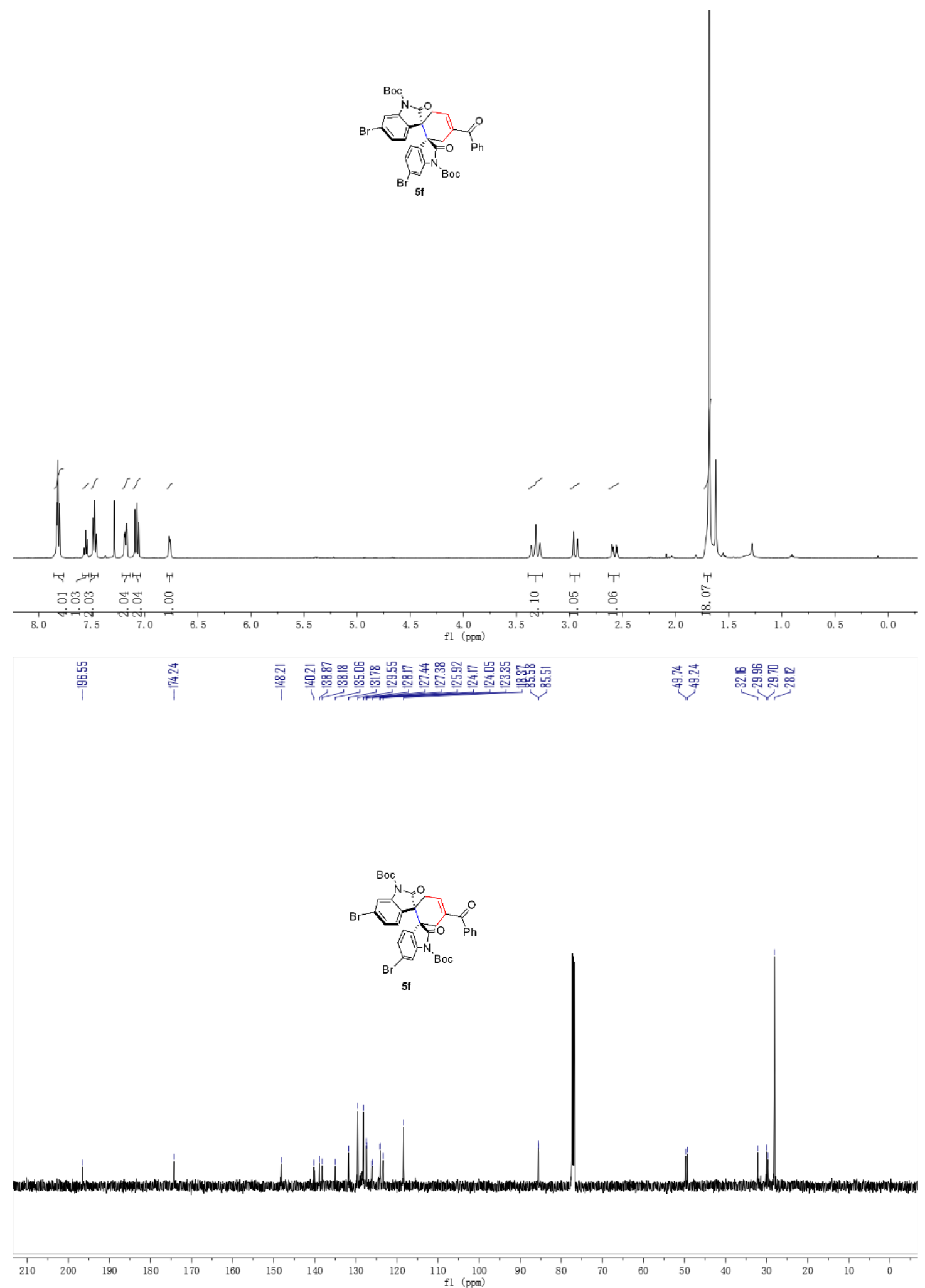


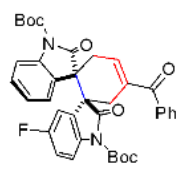

$5 \mathrm{~g}$

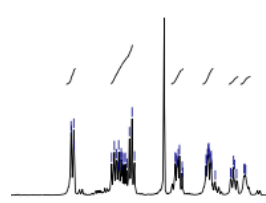
ith when in

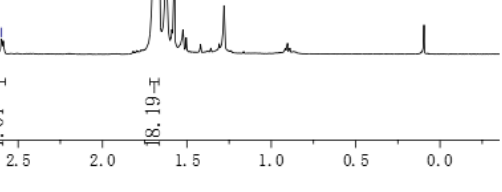

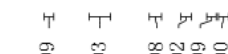

ד

ร

$8.0=\frac{1}{7.5}+\frac{100}{7.0}$

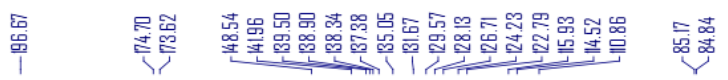

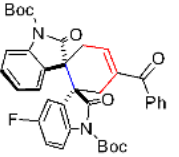

$5 \mathrm{~g}$

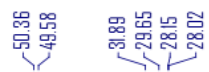

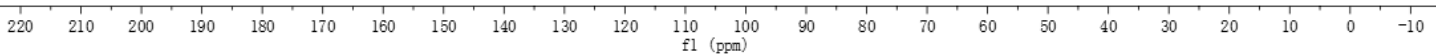




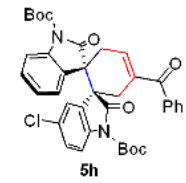

1,

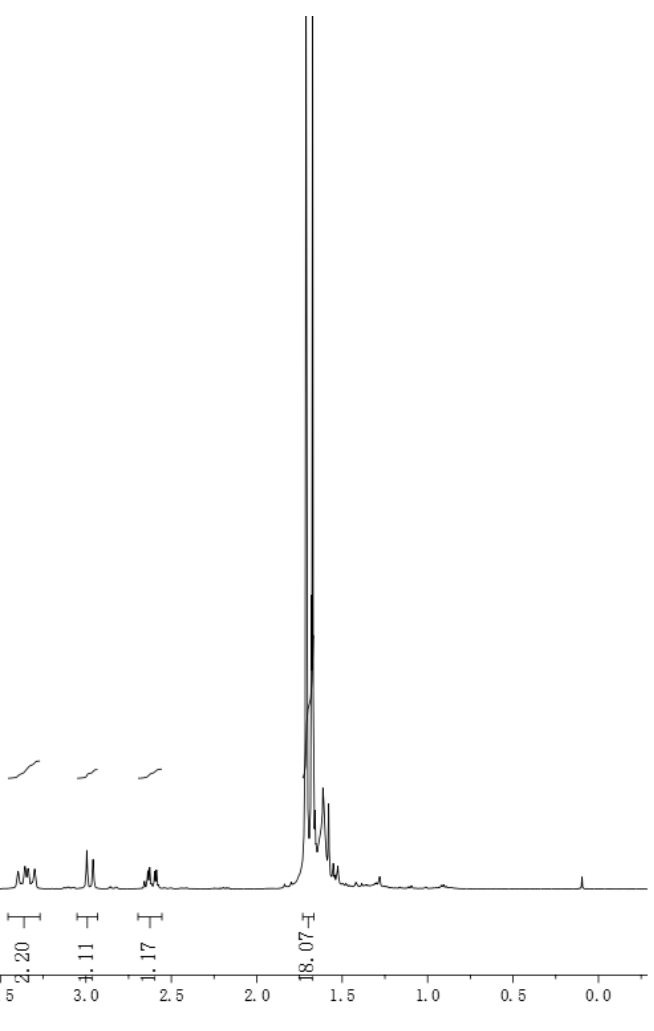

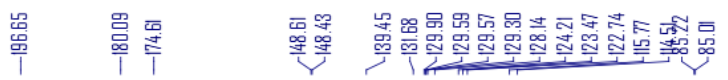

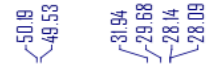

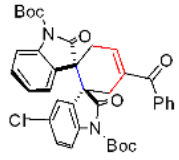

$5 \mathrm{~h}$

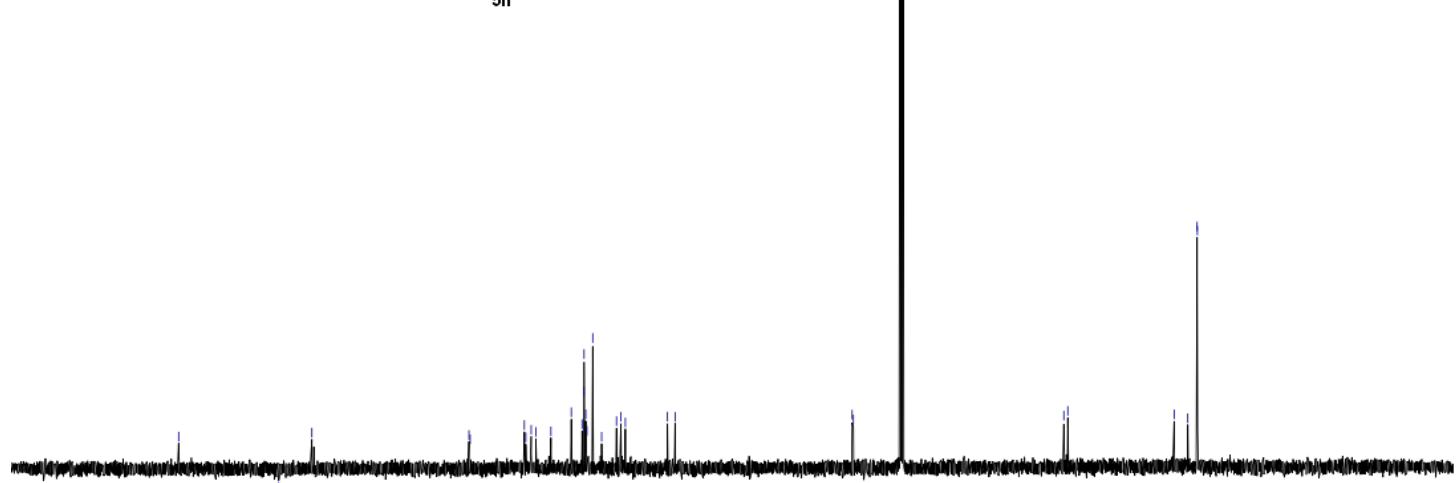

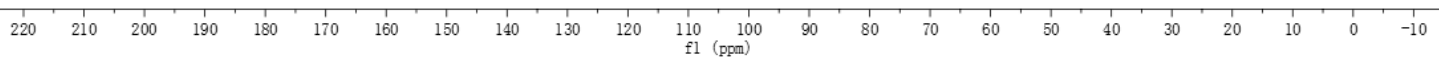




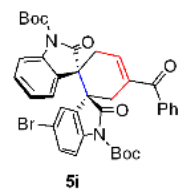

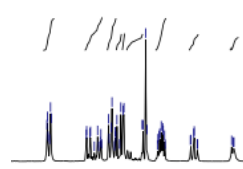

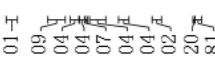

î

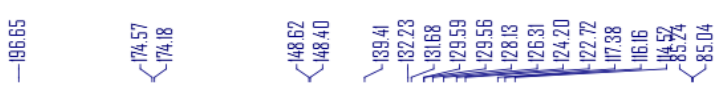

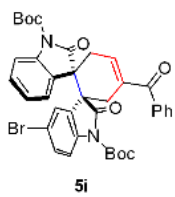

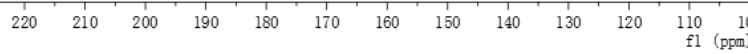



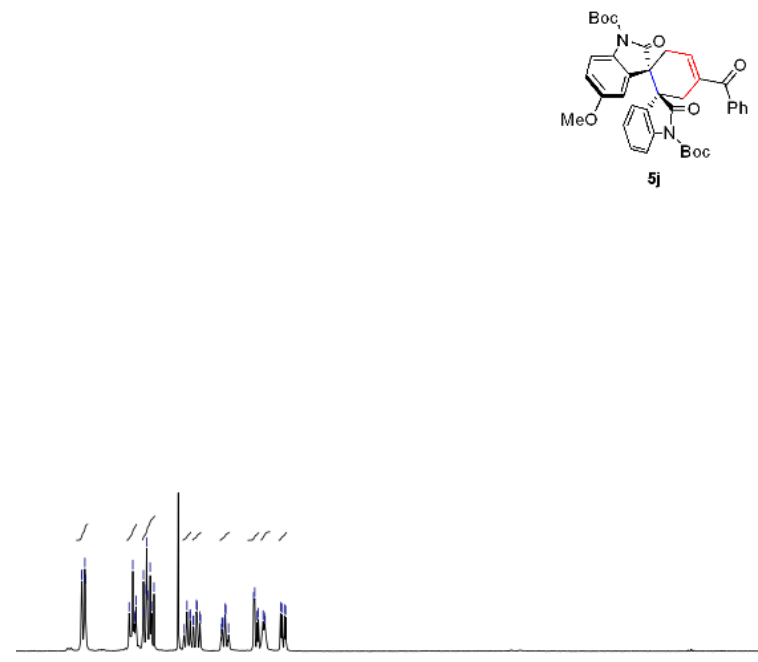

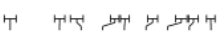
๖

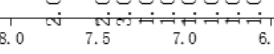

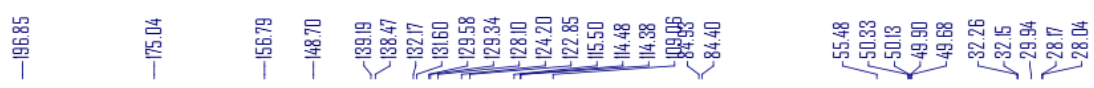

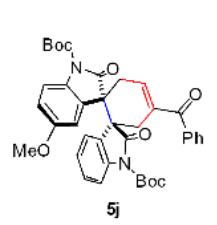

at in is

此 T 은 5 เ

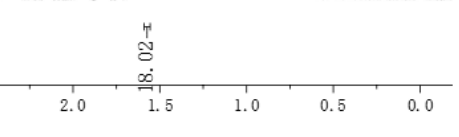

\begin{tabular}{rrrrrrrrrrrrr}
\hline \\
\hline 220 & 210 & 200 & 190 & 180 & 170 & 160 & 150 & 140 & 130 & 120 & 110 & 100 \\
f1 (ppm)
\end{tabular} 


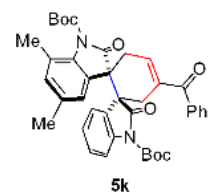

$\mid$

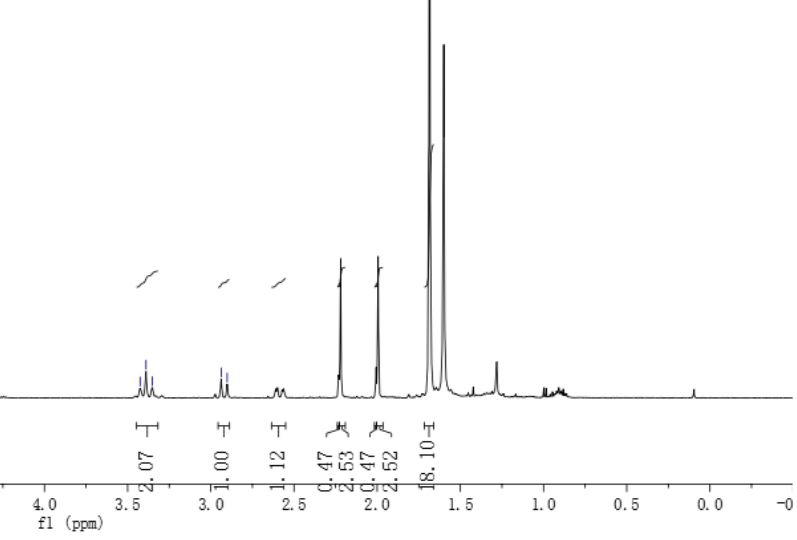

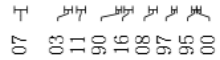

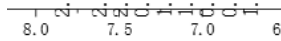

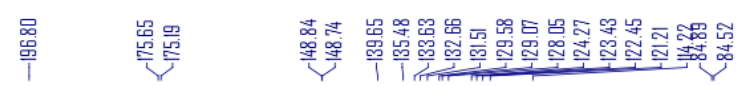

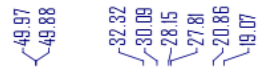

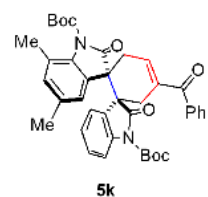

5k

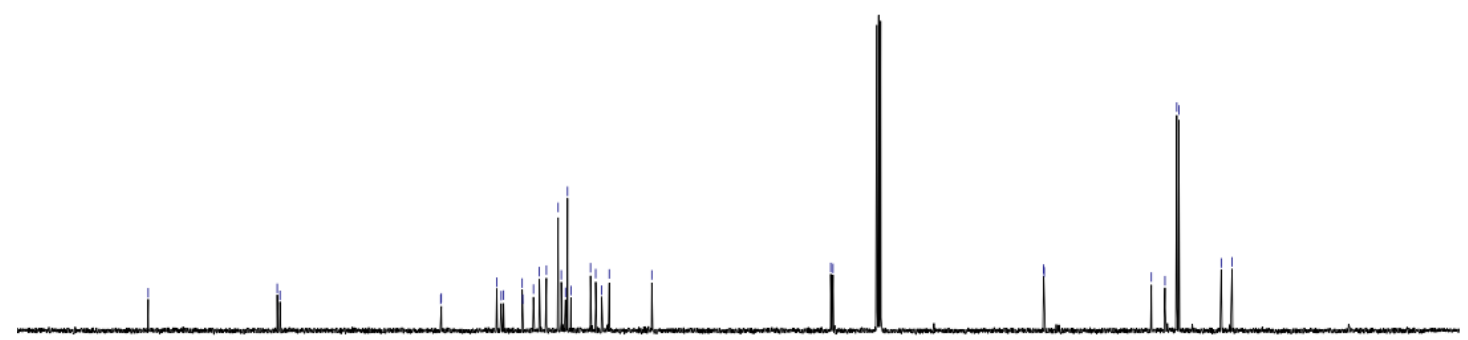

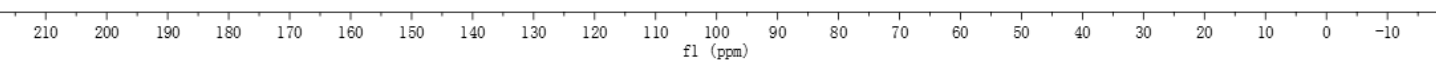



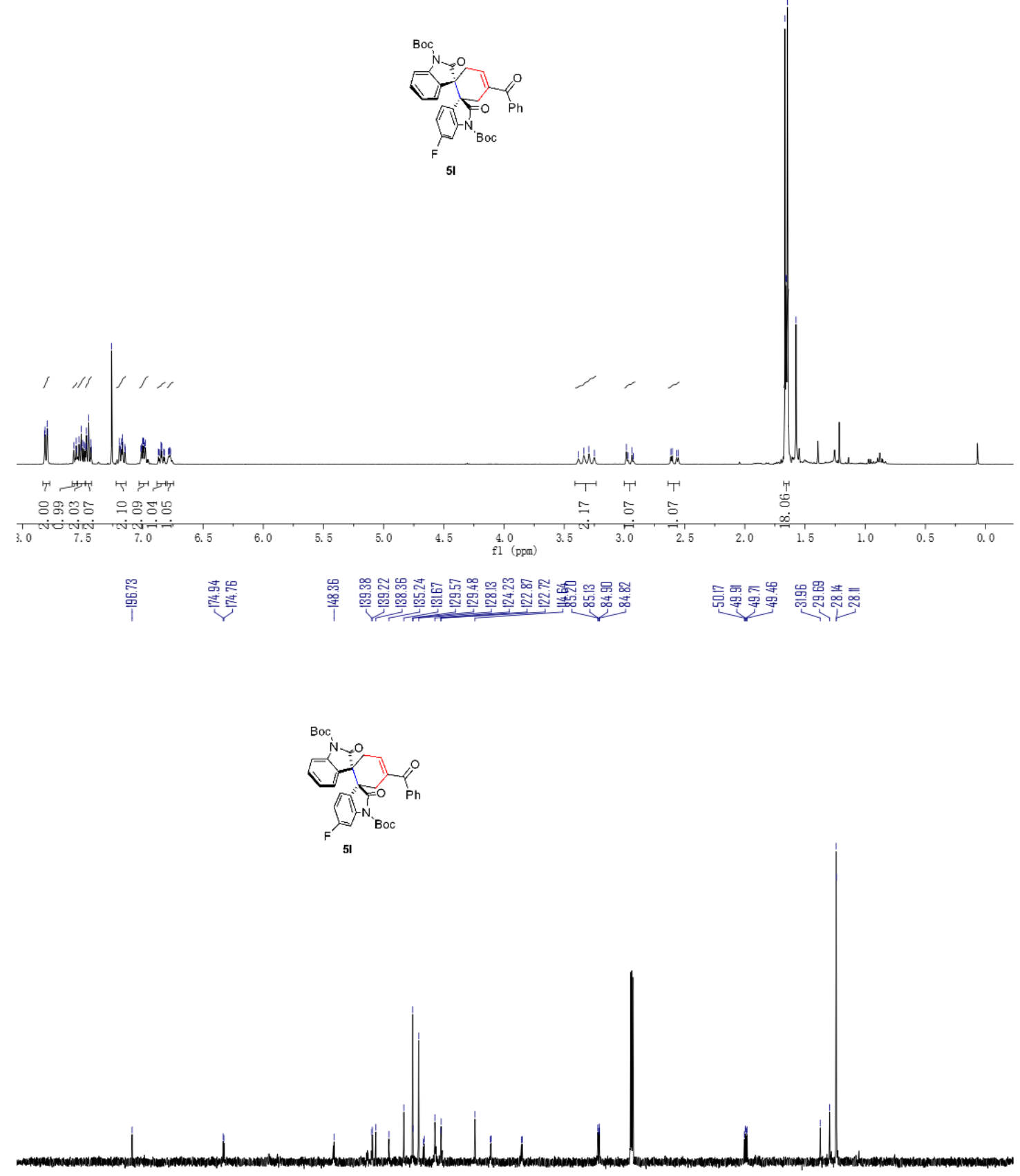

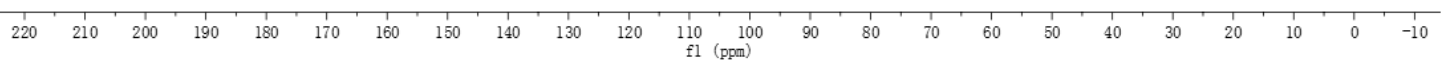




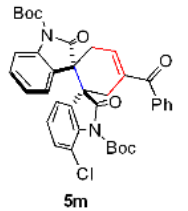

LMhor ili. in

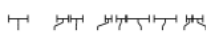

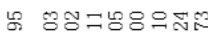
$\begin{array}{lll}\text { 丁 } & \text { ヤ } \\ 5 & 0 & 5\end{array}$

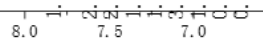

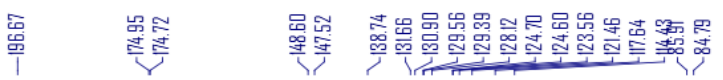

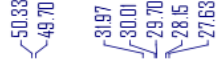

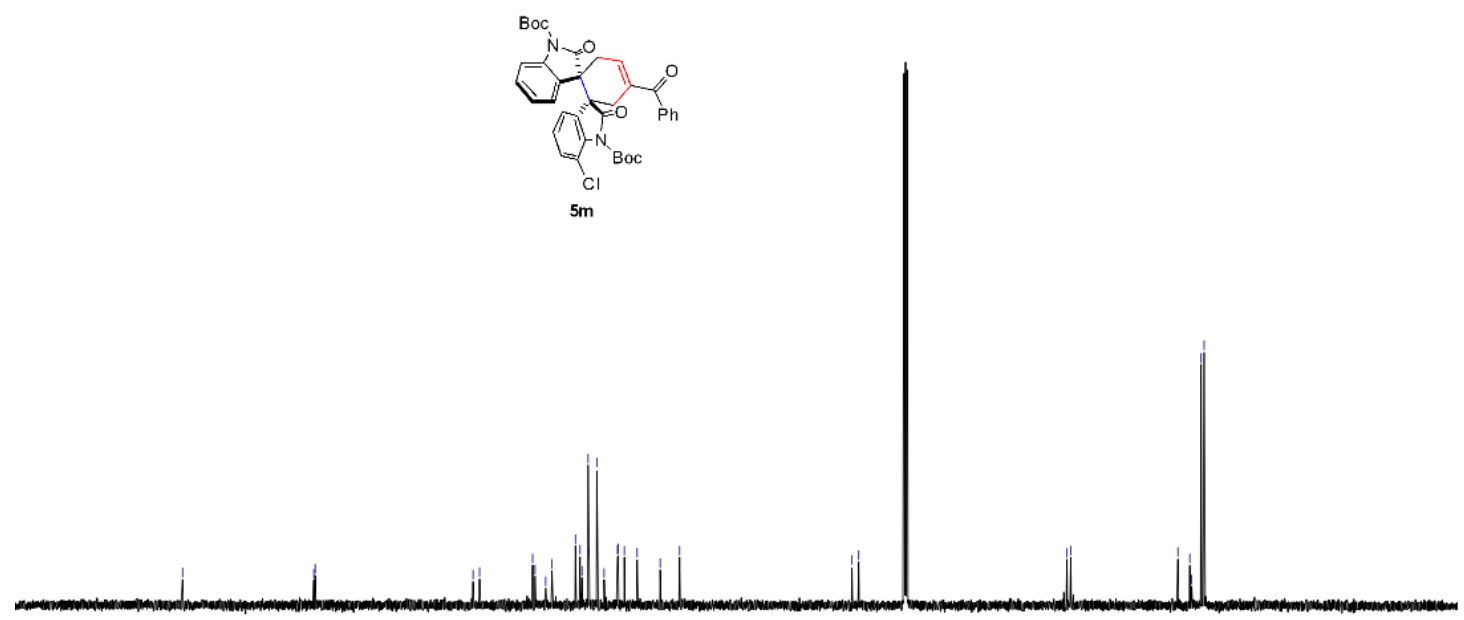

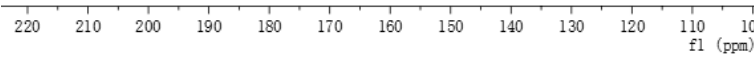



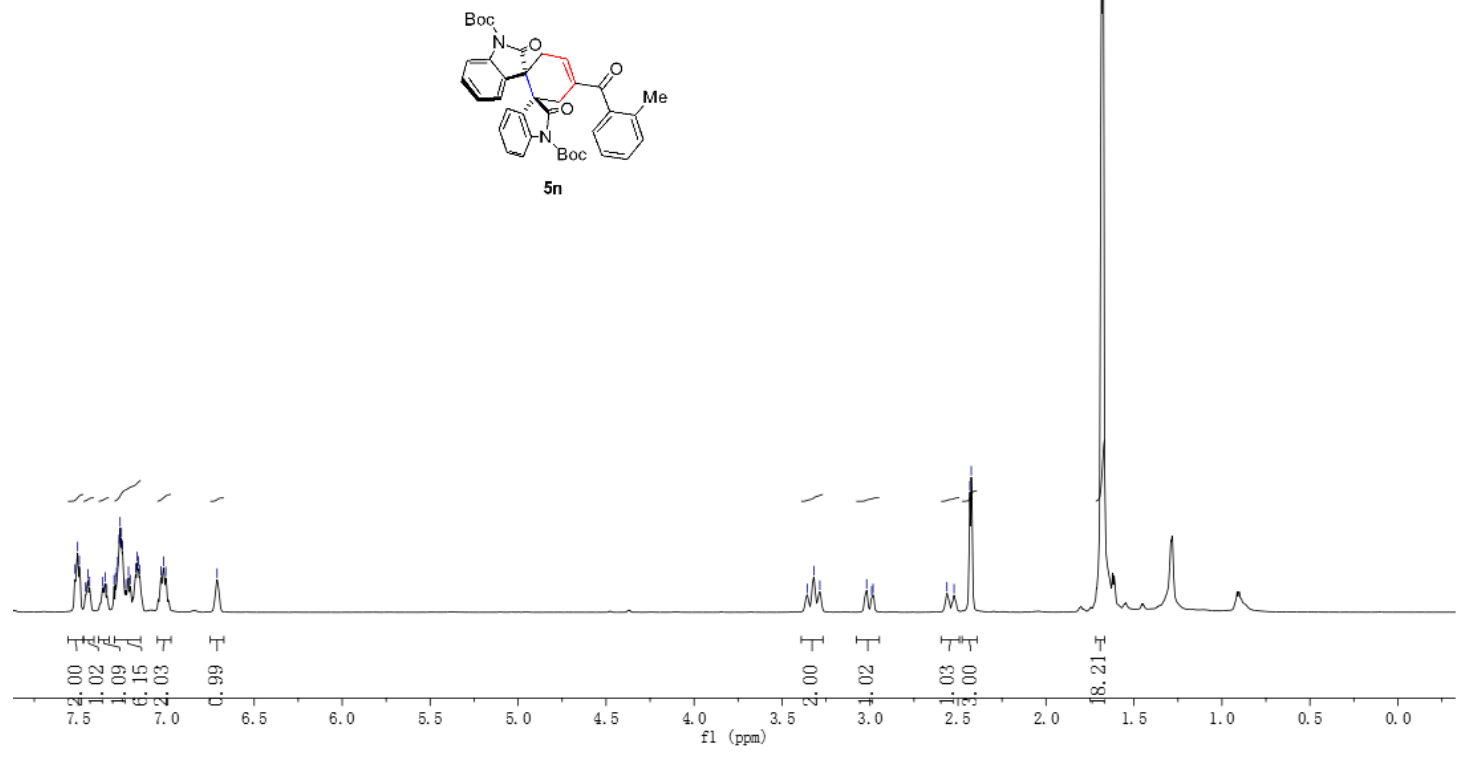

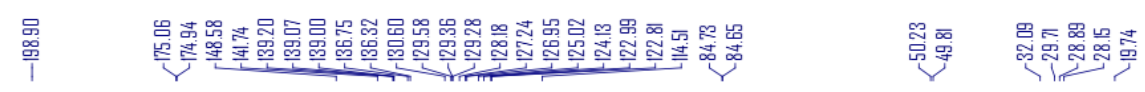

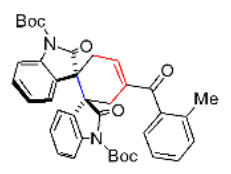

$5 n$

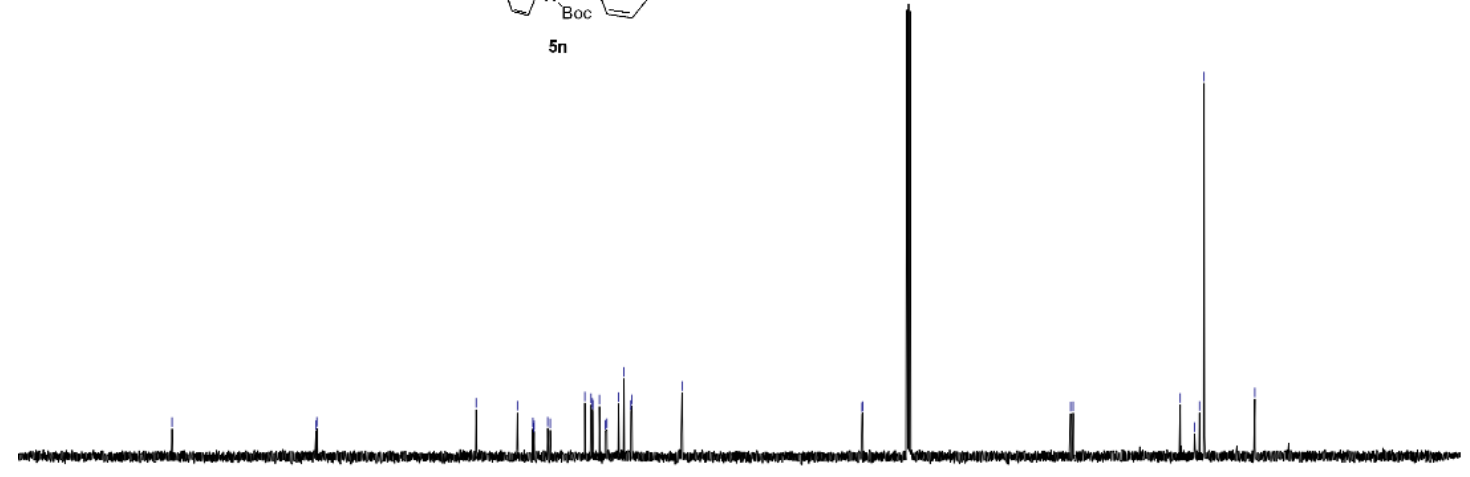

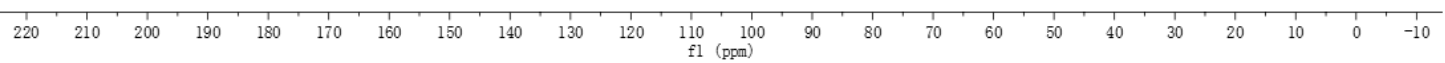



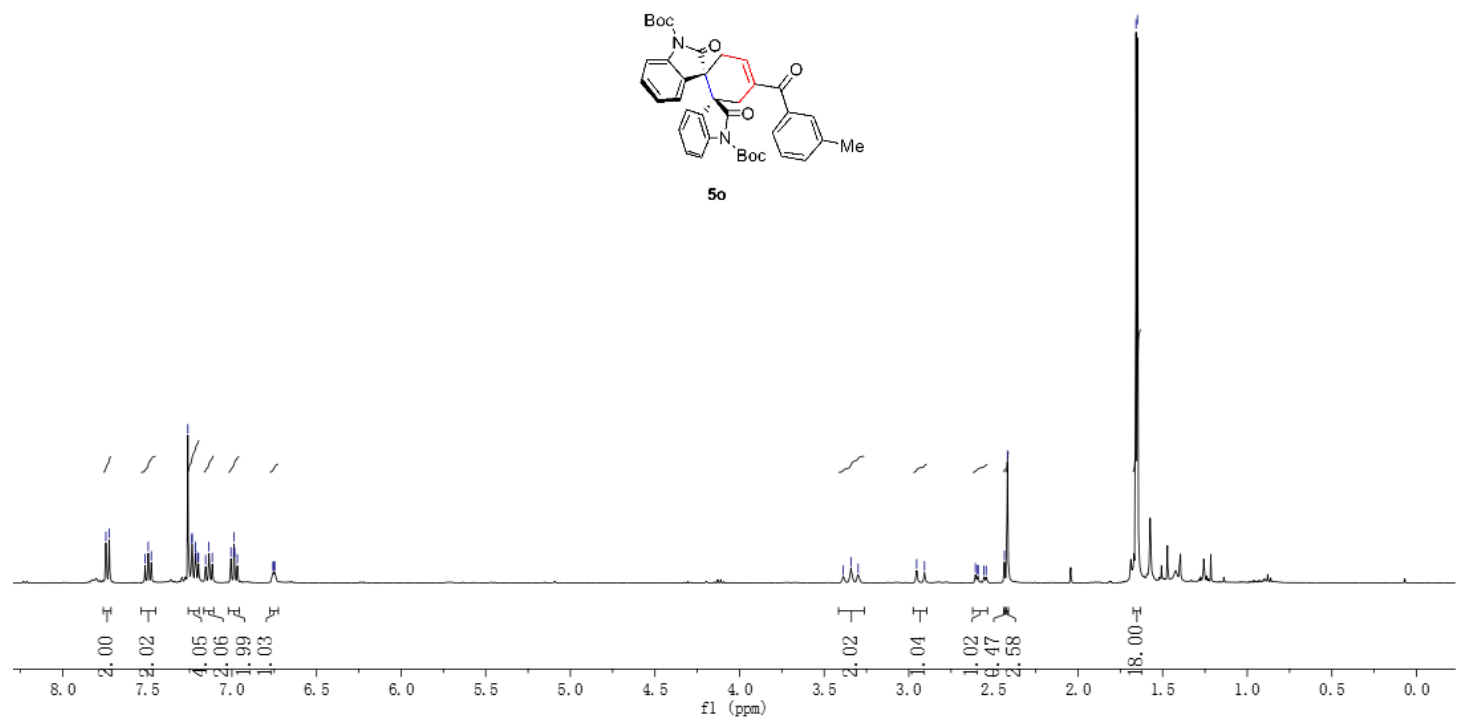

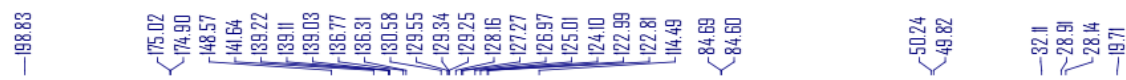
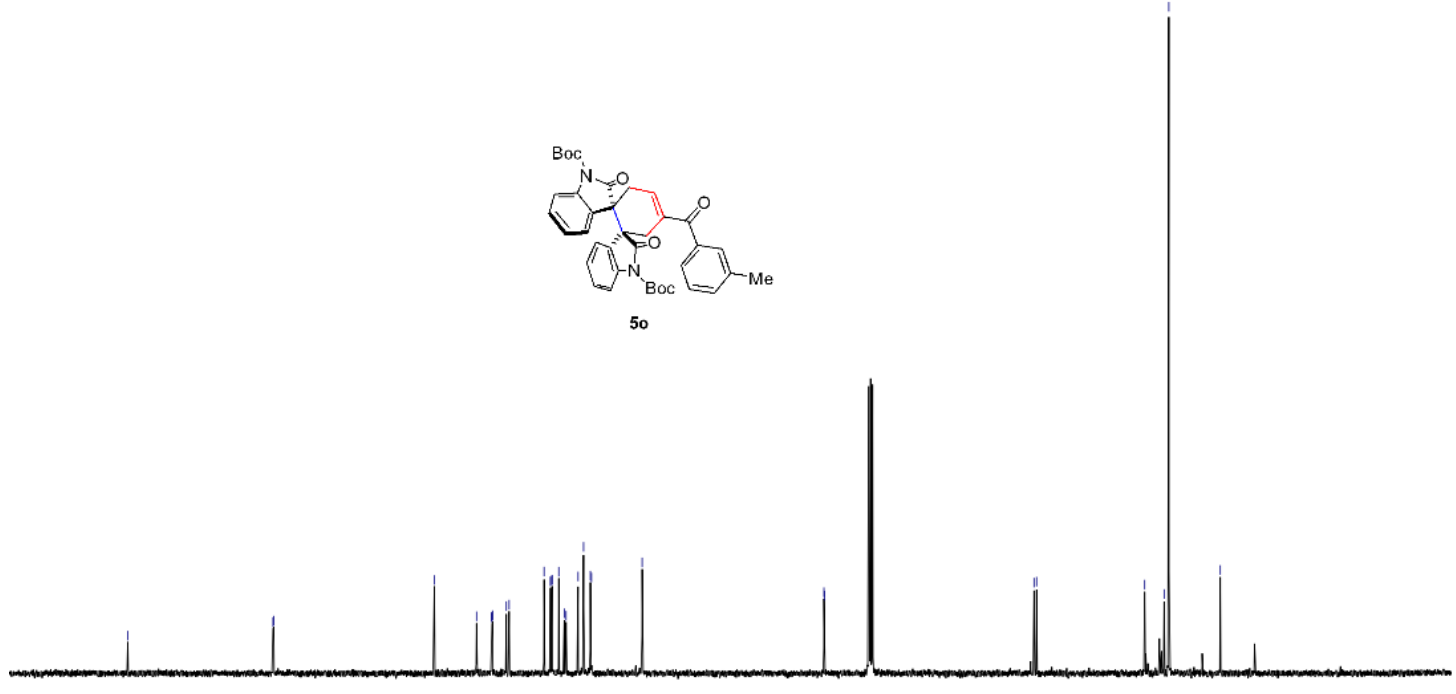

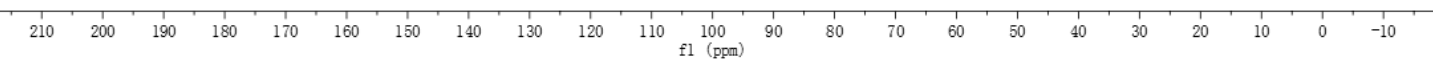



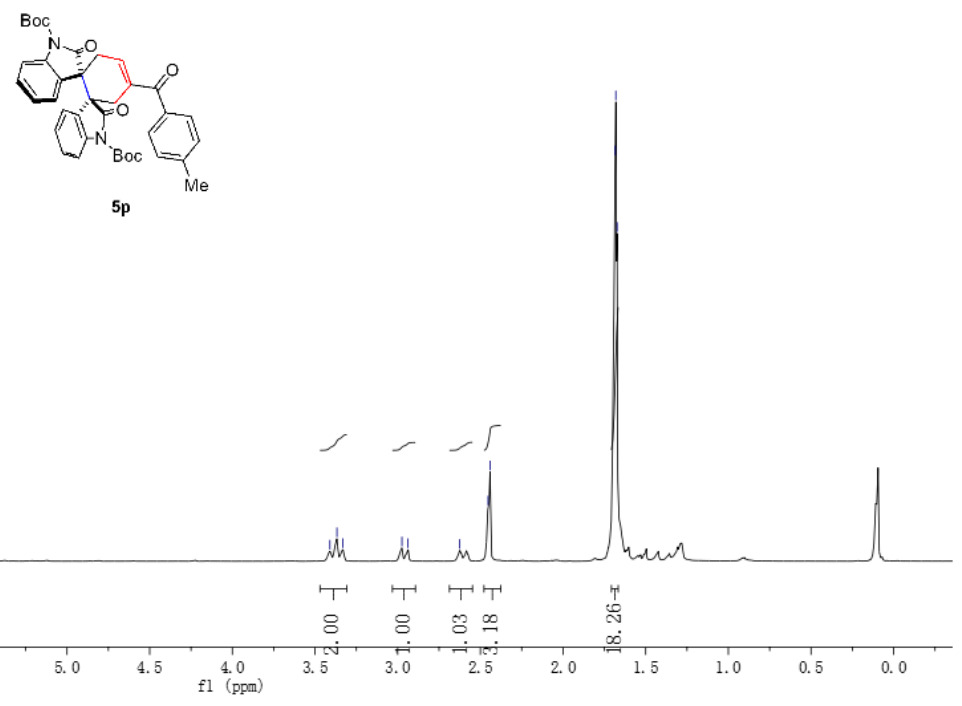

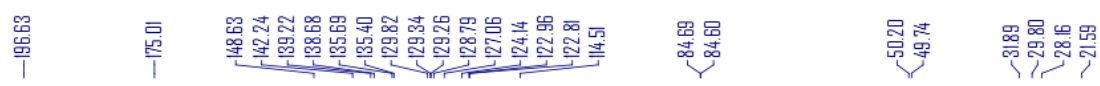

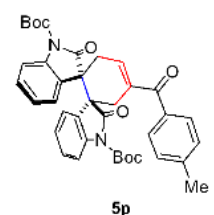

$5 p$

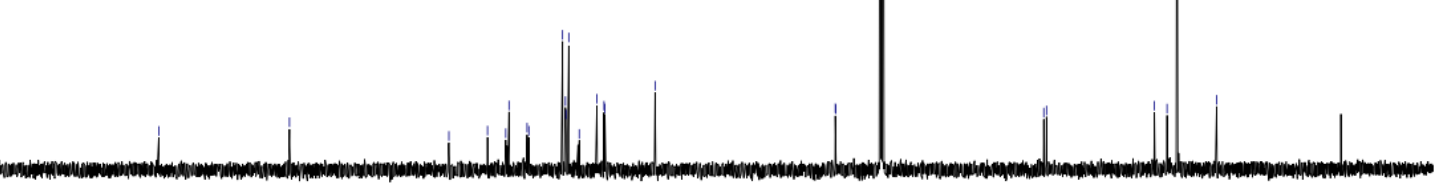

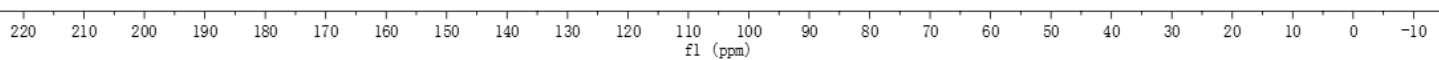



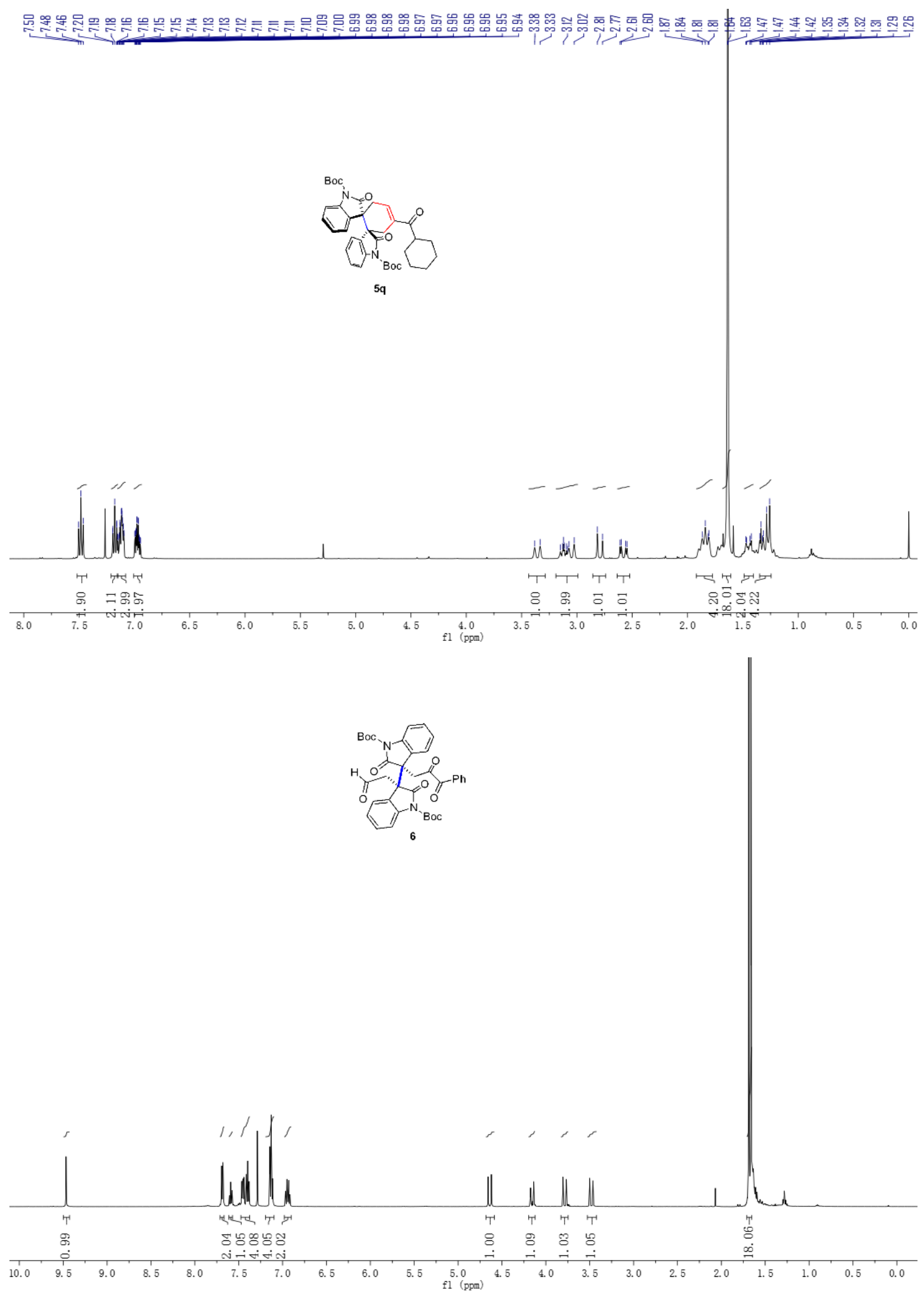


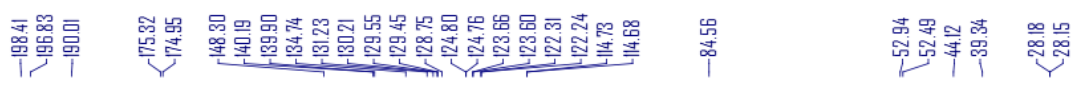
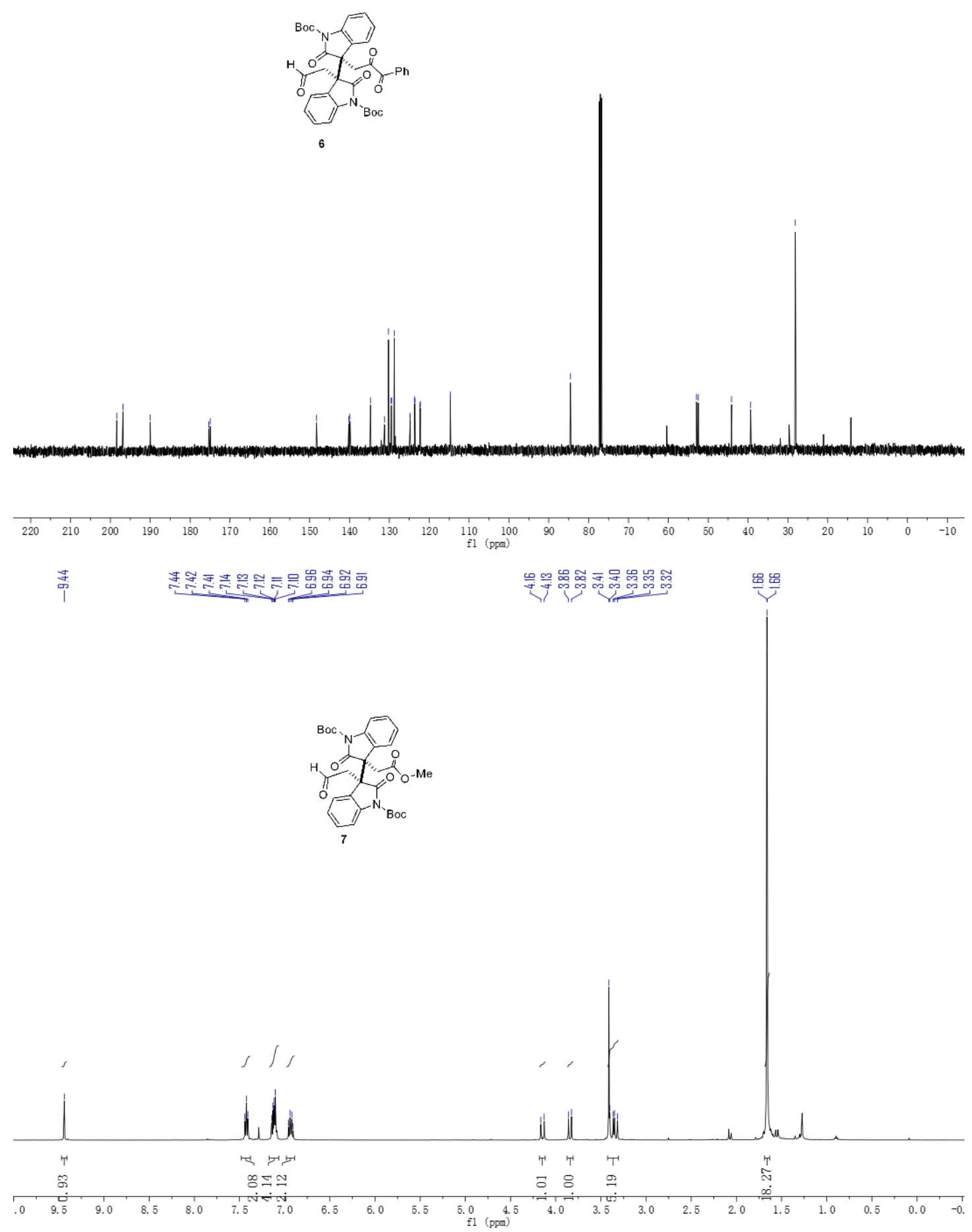

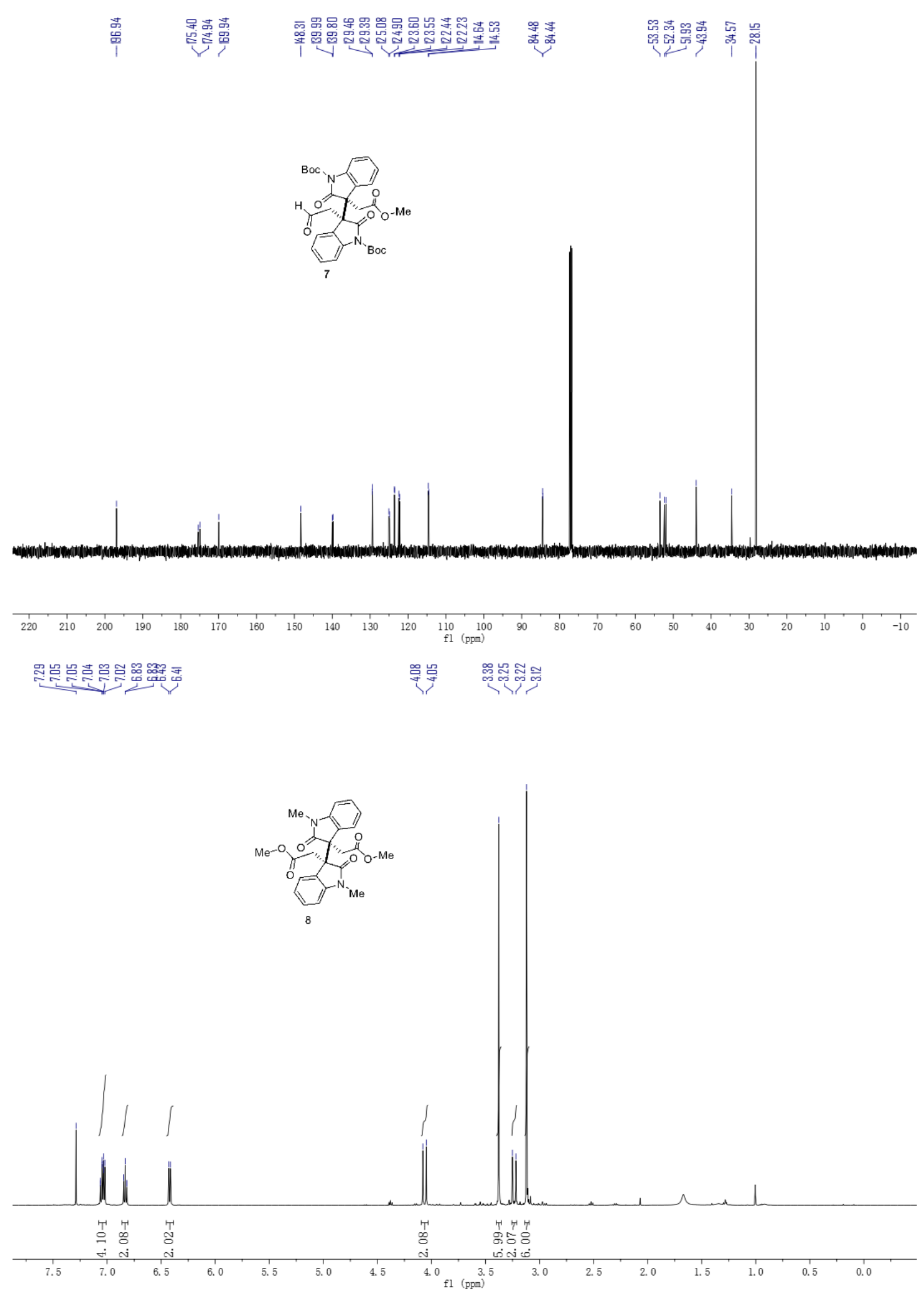


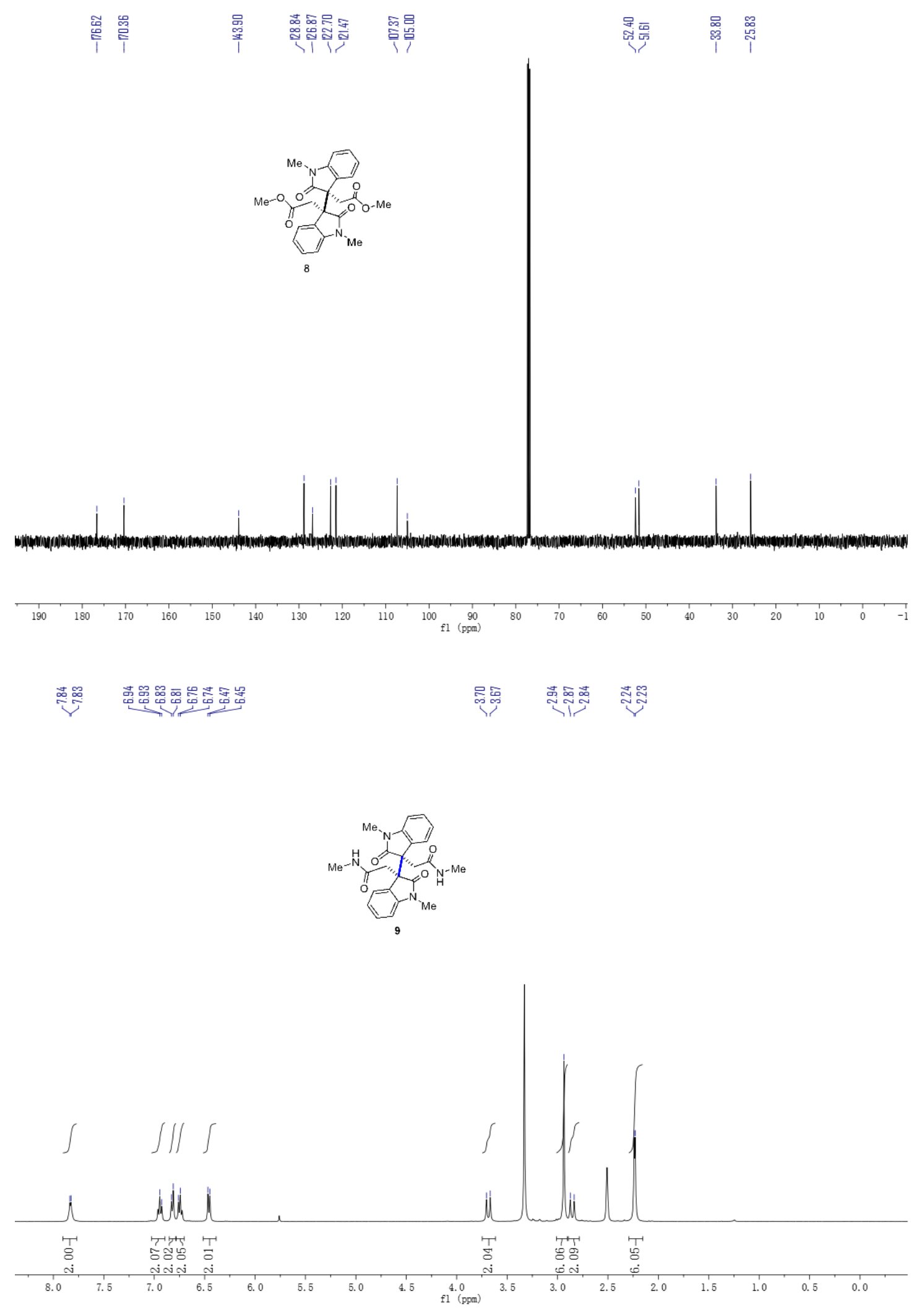



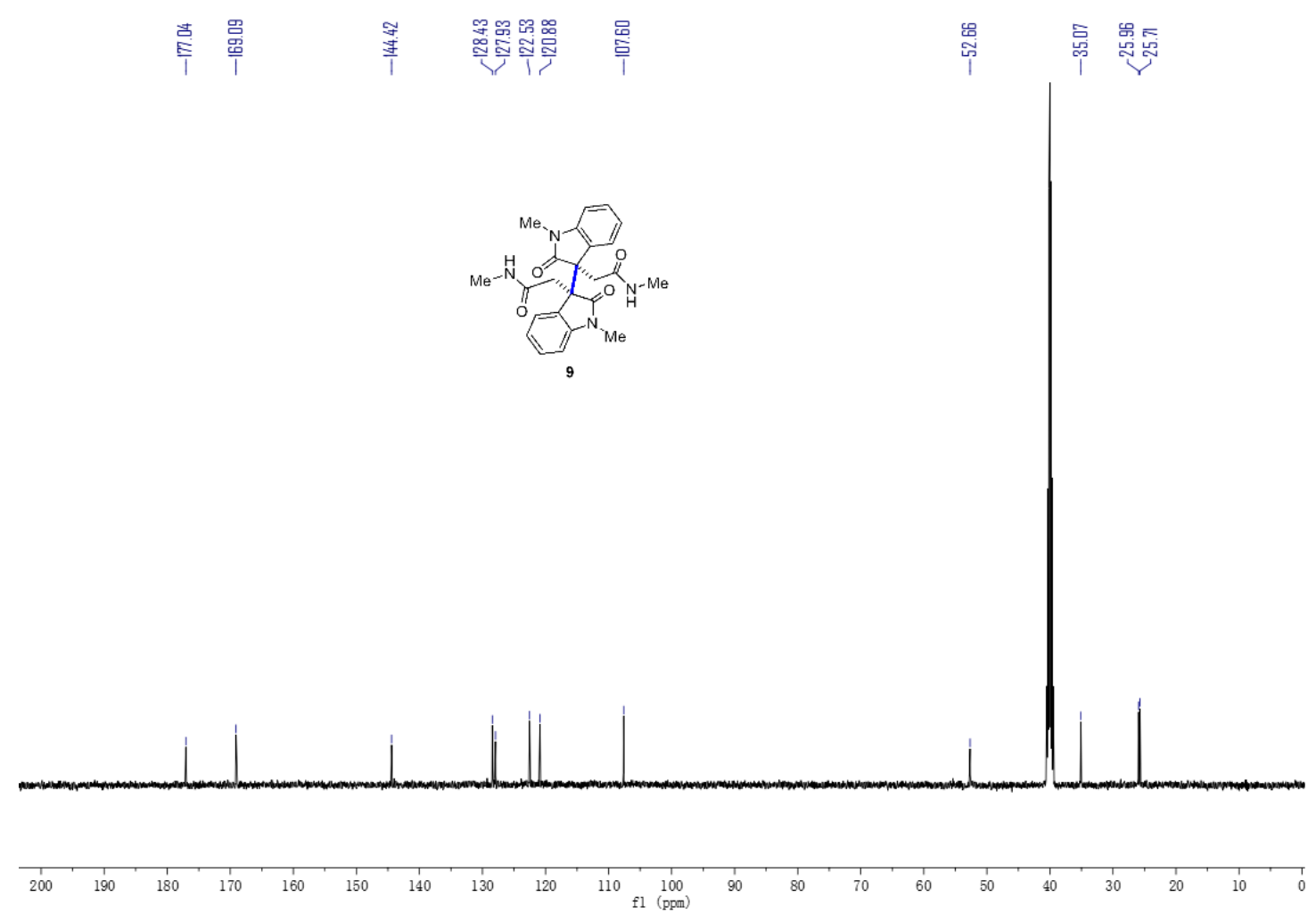

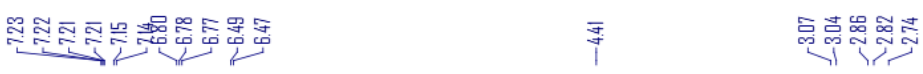

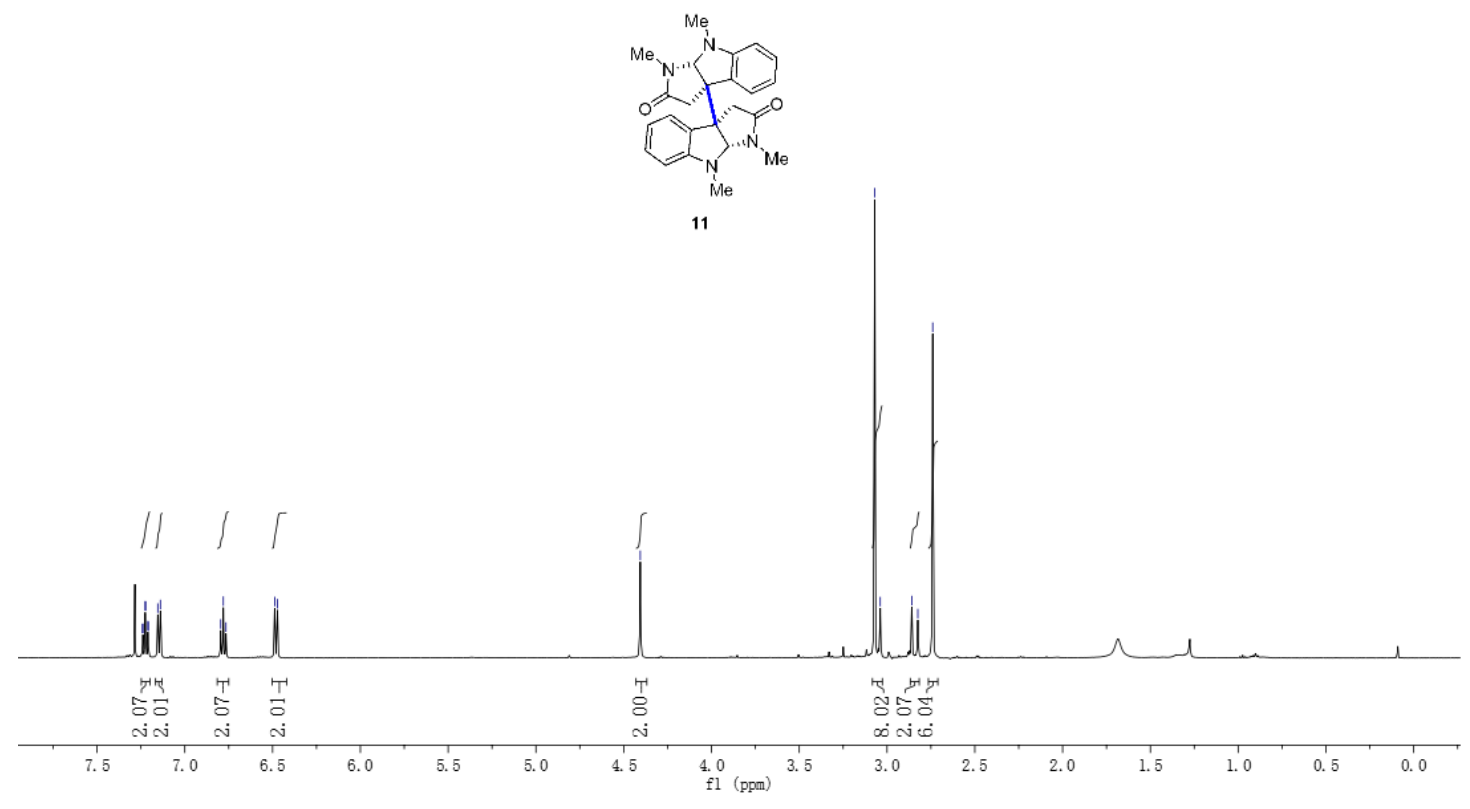




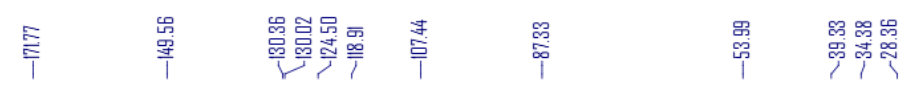

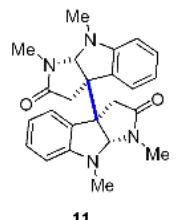

11

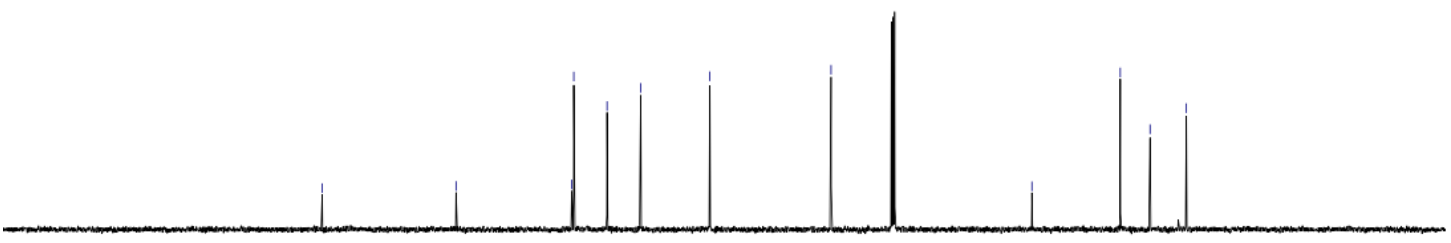

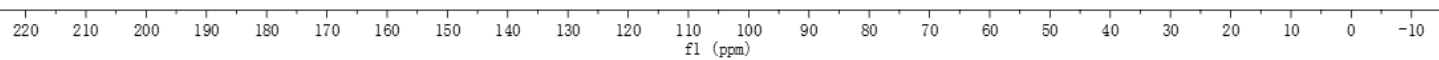



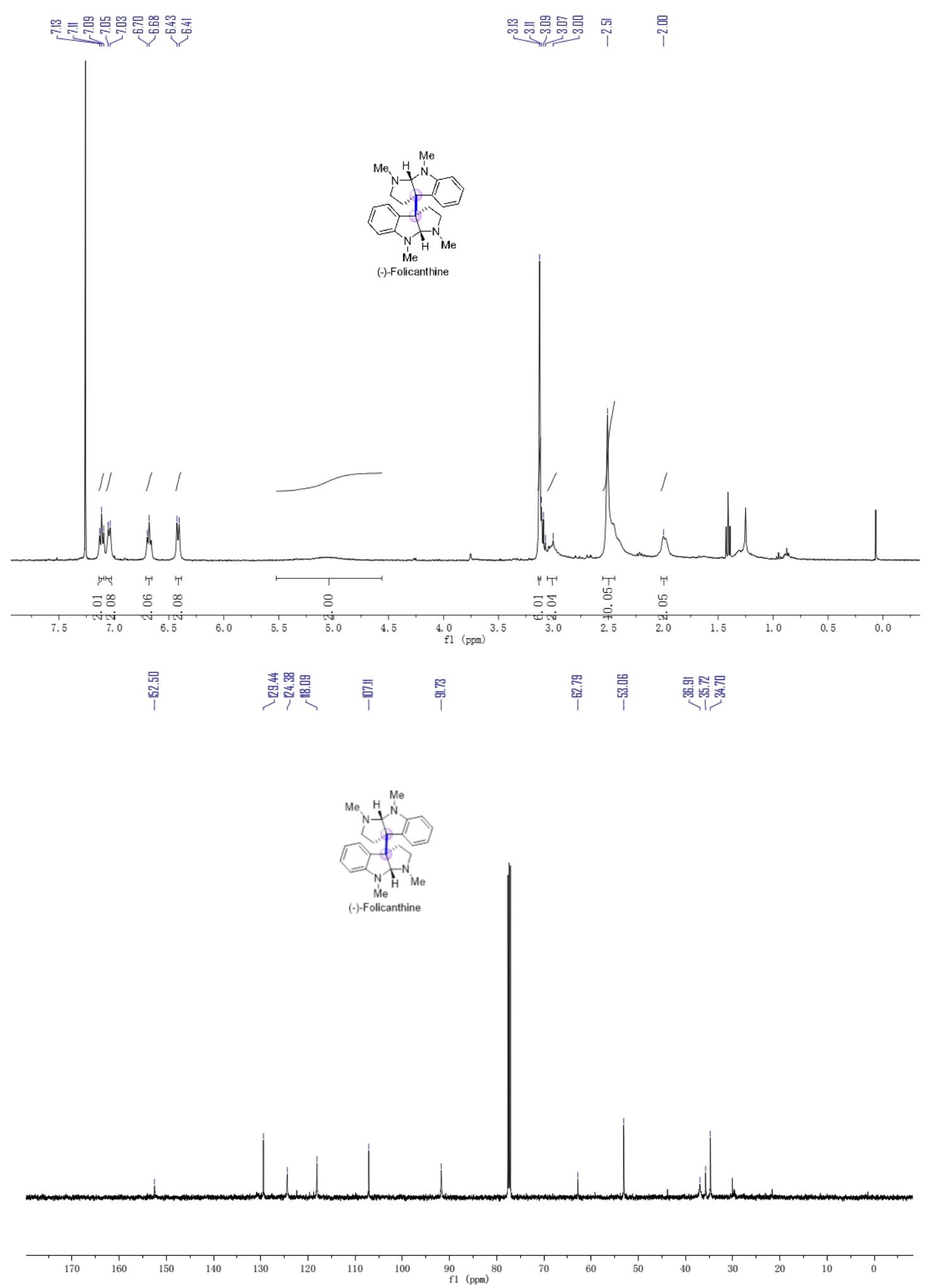


\section{REFERENCES}

(1) Han, X.; Wang, Y.; Zhong, F.; Lu, Y. Enantioselective [3 + 2] Cycloaddition of Allenes to Acrylates Catalyzed by Dipeptide-Derived Phosphines: Facile Creation of Functionalized Cyclopentenes Containing Quaternary Stereogenic Centers. J. Am. Chem. Soc. 2011, 133, 1726-1729.

(2) Chen, S. K.; Ma, W. Q.; Yan, Z. B.; Zhang, F. M.; Wang, S. H.; Tu, Y. Q.; Zhang, X. M.; Tian, J. M. Organo-Cation Catalyzed Asymmetric Homo/Heterodialkylation of Bisoxindoles: Construction of Vicinal All-Carbon Quaternary Stereocenters and Total Synthesis of (-)-Chimonanthidine. J. Am. Chem. Soc. 2018, 140, 10099-10103. (3) Guo, C.; Song, J.; Huang, J. Z.; Chen, P. H.; Luo, S. W.; Gong, L. Z. Core-StructureOriented Asymmetric Organocatalytic Substitution of 3-Hydroxyoxindoles: Application in the Enantioselective Total Synthesis of (+)-Folicanthine. Angew. Chem., Int. Ed. 2012, 51, 1046-1050.

(4) Ghosh, S.; Chaudhuri, S.; Bisai, A. Catalytic Enantioselective Decarboxylative Allylations of a Mixture of Allyl Carbonates and Allyl Esters: Total Synthesis of (-)and (+)-Folicanthine. Chem. Eur. J. 2015, 21, 17479-17484.

(5) Kurita, T.; Aoki, F.; Mizumoto, T.; Maejima, T.; Esaki, H.; Maegawa, T.; Monguchi, Y.; Sajiki, H. Facile and Convenient Method of Deuterium Gas Generation Using a $\mathrm{Pd} / \mathrm{C}$-Catalyzed $\mathrm{H}_{2}-\mathrm{D}_{2}$ Exchange Reaction and Its Application to Synthesis of Deuterium-Labeled Compounds. Chem. Eur. J. 2008, 14, 3371-3379. 